\title{
Analyses of the Life Cycle of TLC1 and the Nuclear RNA Quality Control System in Saccharomyces cerevisiae
}

\author{
Dissertation \\ for the award of the degree \\ “Doctor of Philosophy” (Ph.D.) \\ Division of Mathematics and Natural Sciences \\ of the Georg-August-Universität Göttingen \\ within the basic program biology
}

of the Georg-August University School of Science (GAUSS)

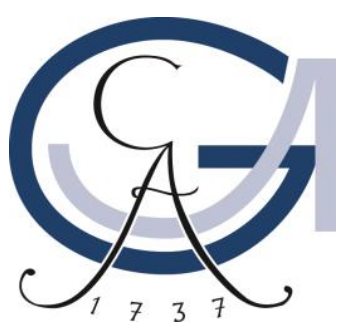

submitted by

Haijia Wu

Born in Wuhan, China

Göttingen, March 2015 


\section{Members of the Thesis Committee}

\section{Prof. Dr. Heike Krebber}

Department of Molecular Genetics, Institute of Microbiology and Genetics, Georg-August University Göttingen

\section{PD Dr. Wilfried Kramer}

Department of Molecular Genetics, Institute of Microbiology and Genetics, Georg-August University Göttingen

\section{Members of the Examination Board}

Prof. Dr. Heike Krebber ( $1^{\text {st }}$ reviewer $)$

Department of Molecular Genetics, Institute of Microbiology and Genetics, Georg-August University Göttingen

PD Dr. Wilfried Kramer (2 ${ }^{\text {nd }}$ reviewer)

Department of Molecular Genetics, Institute of Microbiology and Genetics, Georg-August University Göttingen

\section{Prof. Dr. Gerhard Braus}

Department of Molecular Microbiology and Genetics, Institute of Microbiology and Genetics, Georg-August University Göttingen

\section{Prof. Dr. Ralf Ficner}

Department of Molecular Structural Biology, Institute of Microbiology and Genetics, Georg-August University Göttingen

\section{Prof. Dr. Kai Heimel}

Department of Molecular Microbiology and Genetics, Institute of Microbiology and Genetics, Georg-August University Göttingen

\section{Prof. Dr. Stefanie Pöggeler}

Department of Genetics of Eukaryotic Microorganisms, Institute of Microbiology and Genetics, Georg-August University Göttingen

Date of oral examination: 23. April.2015 


\section{Declaration}

I hereby confirm that this thesis entitled:

"Analyses of the Life Cycle of TLC1 and the Nuclear RNA Quality Control System in Saccharomyces cerevisiae"

has been written independently and with no other sources and aids than quoted.

Haijia Wu

Göttingen, March, 2015

Parts of this work were published in:

Wu, H., Becker, D., and Krebber, H. (2014). Telomerase RNA TLC1 shuttling to the cytoplasm requires mRNA export factors and is important for telomere maintenance. Cell reports $8,1630-1638$. 


\section{Table of Contents}

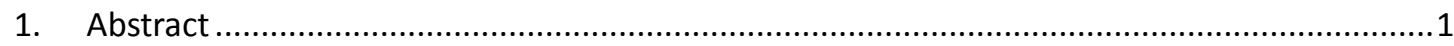

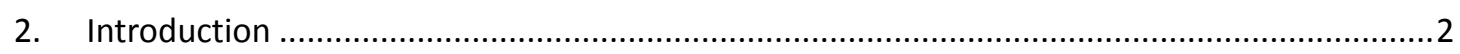

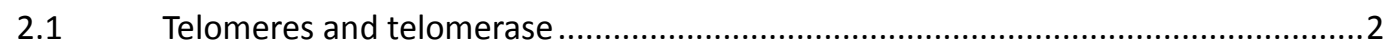

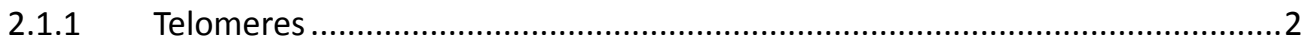

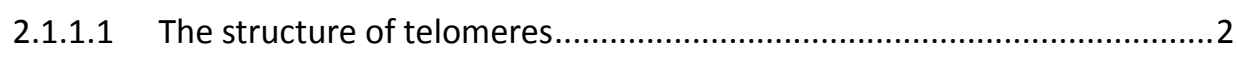

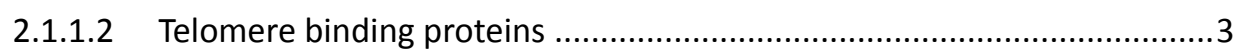

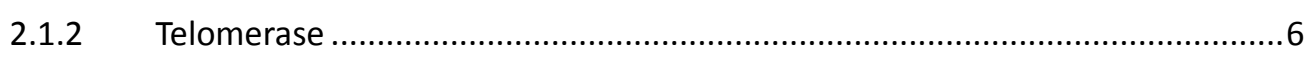

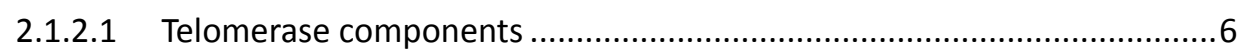

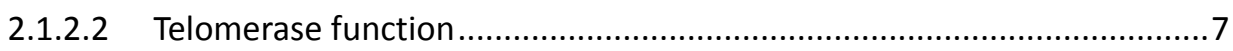

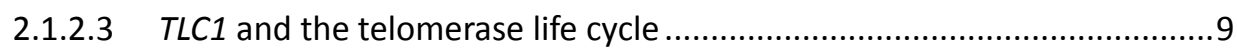

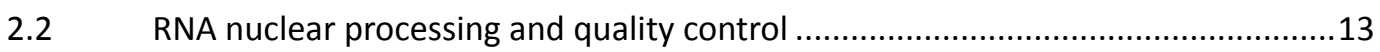

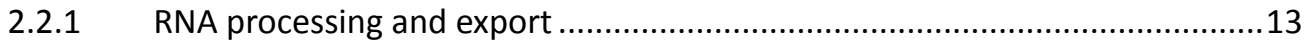

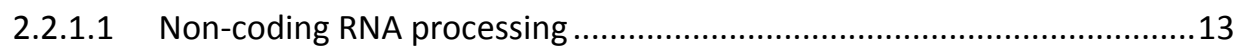

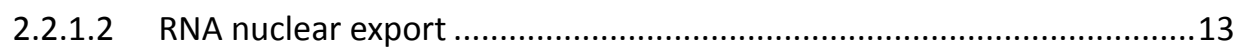

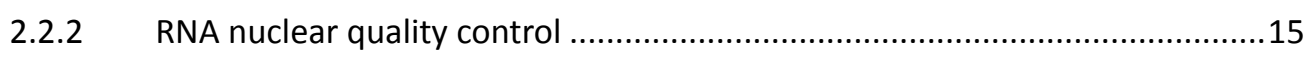

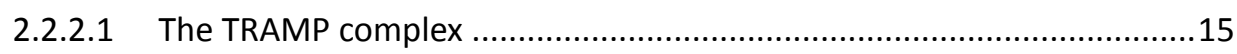

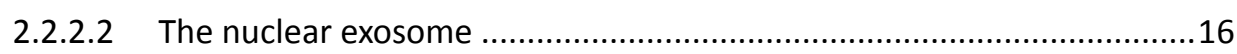

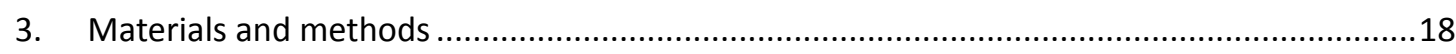

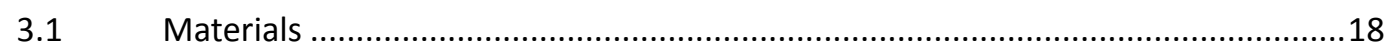

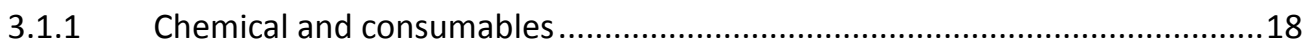

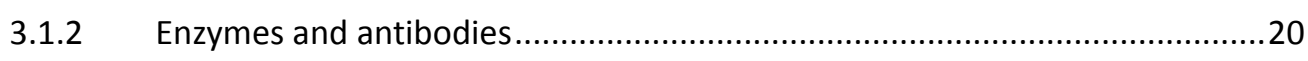

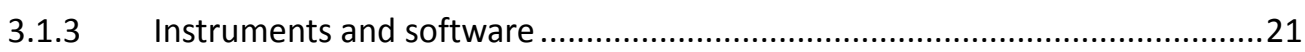

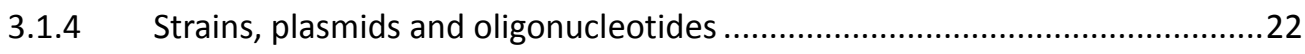

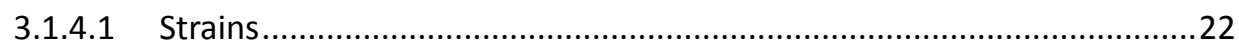

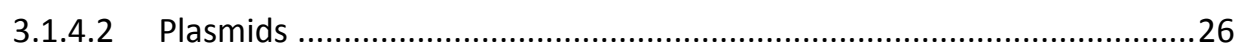

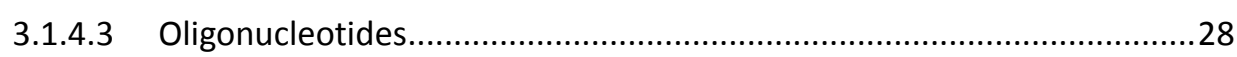

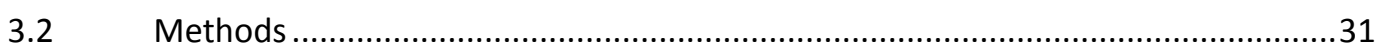

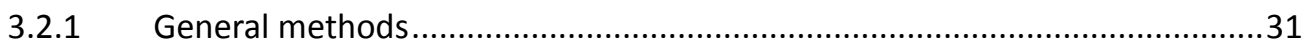

3.2.1.1 Generation of strains, plasmids and oligonucleotides ..........................31

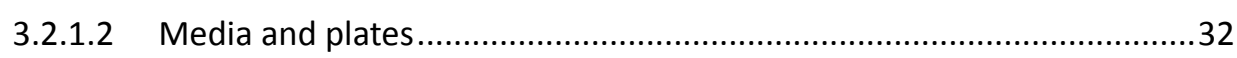

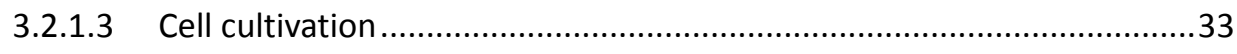

3.2.1.4 Yeast sporulation and tetrad analysis.....................................................

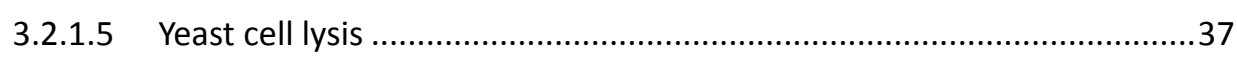

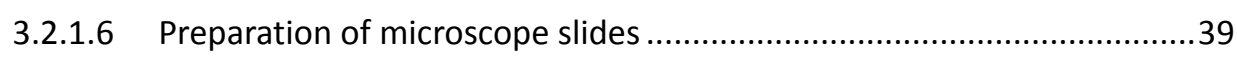

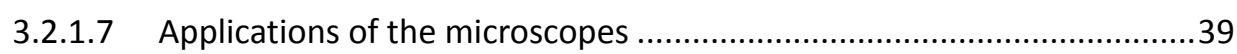

3.2.1.8 Signal detection, quantification and statistical analyses ...........................40

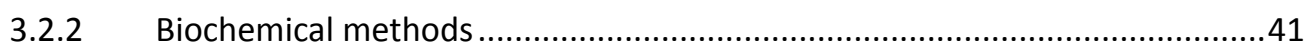

3.2.2.1 Protein extraction and precipitation .................................................... 41

3.2.2.2 Co-immunoprecipitation (IP or co-IP) ................................................4

3.2.2.3 Nucleo-cytoplasmic fractionation ..........................................................4

3.2.2.4 SDS-polyacrylamide gel electrophoresis (SDS-PAGE) and western blot...44 
3.2.3 Molecular biological methods

3.2.3.1 Polymerase chain reaction (PCR) ................................................... 47

3.2.3.2 DNA gel electrophoresis and gel extraction ........................................48

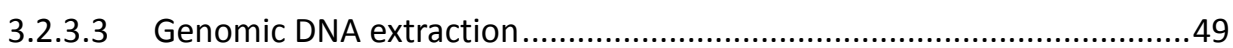

3.2.3.4 Acidic phenol RNA extraction .........................................................50

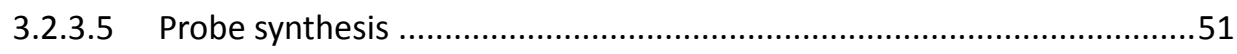

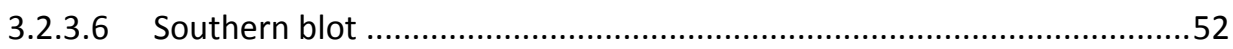

3.2.3.7 RNA-co-immunoprecipitation (RNA-CO-IP) .......................................54

3.2.3.8 Quantitative reverse transcriptase PCR (qRT-PCR) ................................55

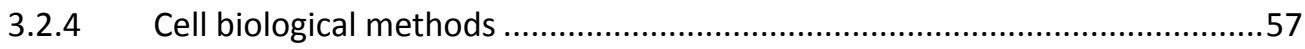

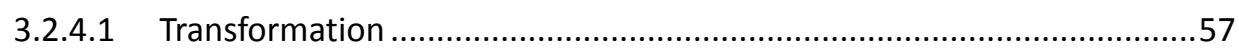

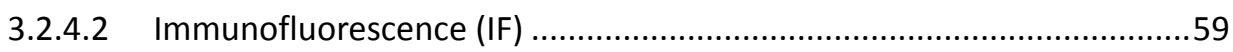

3.2.4.3 RNA fluorescent in situ hybridization (FISH) ....................................60

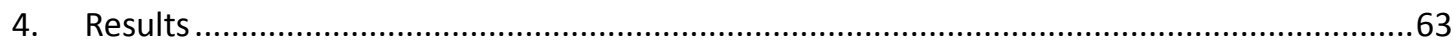

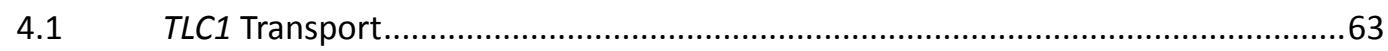

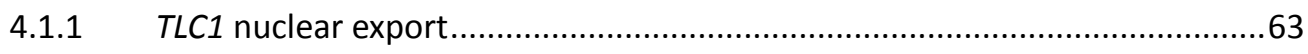

4.1.1.1 Fluorescent in situ hybridization experiments reveal that the nuclear export of TLC1 requires the mRNA export machinery ..........................................63

4.1.1.2 Nucleo-cytoplasmic fractionation shows decreased cytoplasmic abundance of TLC1 upon blocking the mRNA export pathway .............................67

4.1.1.3 The mRNA export factors physically interact with $T L C 1$.......................69

4.1.1.4 The TLC1 nuclear export block observed in mRNA export mutants is not due to impaired TLC1 transcription or maturation ................................................. 71

4.1.1.5 The TLC1 nuclear export is mediated through cooperation of Xpo1/Crm1

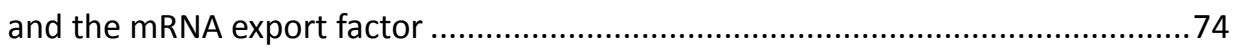

4.1.2 A TLC1 export block affects telomerase formation ........................................76

4.1.2.1 TLC1 cytoplasmic deficiency leads to impaired localisation of the telomerase components

4.1.2.2 Less $T L C 1$-Est2 interactions are formed due to the $T L C 1$ nuclear retention 81

4.1.2.3 Reduction of the TLC1 cytoplasmic presence affects the interaction between the telomerase components

4.1.3 Analyses of the mex67-5 xpo1-1 double mutant on TLC1 nuclear export ......87

4.1.3.1 The mex67-5 xpo1-1 double mutant shows a stronger TLC1 nuclear accumulation and an increased rate of TLC1 processing

4.1.3.2 The cytoplasmic mislocalisation of a telomerase component is increased in the xpo1-1 mex67-5 double mutant. ..91

4.1.3.3 Telomere maintenance is impaired in the double mutant xpo1-1 mex67-5 93

4.1.3.4 Xpo1/Crm1 is directly involved in $T L C 1$ nuclear export........................96

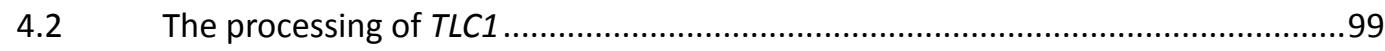

4.2.1 The transcription and processing defects of TLC1 in the mtr10 $\triangle$ strain is not due to its effect on TLC1 transport.

4.2.2 The factors involved in TLC1 processing are identified by qRT-PCR analyses101 
4.2.3 The factors involved in the processing of TLC1 are mis-localised in the mtr10A mutant 105

4.2.4 Mtr10 physically interacts with factors that are involved in the processing of TLC1 107

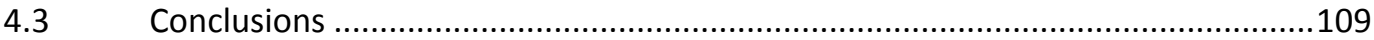

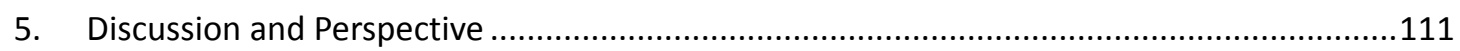

5.1 The life cycle of the telomerase complex..........................................................111

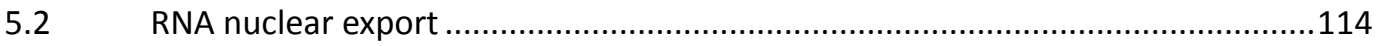

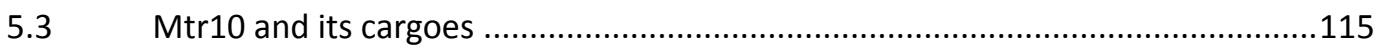

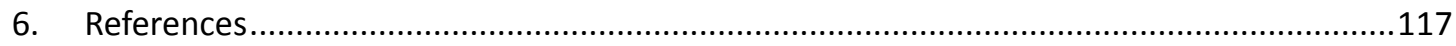

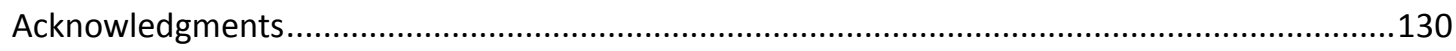

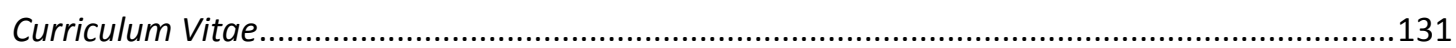




\section{Abstract}

During genome replication, chromosomes undergo a progressive shortening of their ends. To counteract this loss, a complex named telomerase functions as a reverse transcriptase to elongate telomeres. In Saccharomyces cerevisiae, telomerase contains a non-coding RNA, $T L C 1$, which serves as a scaffold for formation of the telomerase complex and the template for the reverse transcription to elongate the telomeres. Upon its synthesis, $T L C 1$ undergoes a series of nuclear and cytoplasmic maturation steps.

Here it has been shown that the TLC1 nuclear export is dependent on the classic mRNA export pathway in addition to the already known Crm1/Xpo1 pathway. The nuclear export defects that occur upon mutation of these pathways impair the formation of the telomerase, as well as its final localisation suggesting an essential role of TLC1 shuttling in telomerase assembly. Consequently, the TLC1 nuclear transport defect leads to telomeric shortening indicating a necessity of the TLC1 shuttling for telomere maintenance. Moreover, the nuclear RNA quality control system, composed of the TRAMP complex and nuclear exosome, might mediate the nuclear maturation of TLC1. Finally, it has also been pointed out that the localisation and maturation of the nuclear quality control system might be regulated by a nuclear importer, Mtr10, which is also involved in TLC1 nuclear import. 


\section{Introduction}

\subsection{Telomeres and telomerase}

\subsubsection{Telomeres}

The genome contains all cellular information important for its own synthesis, cell growth, differentiation and death. This information is transferred from generation to generation through chromosome replication. However, due to the mechanism of DNA replication the linear chromosomes of eukaryotes are not able to be fully duplicated leading to the loss of genomic content during each reproduction cycle (Olovnikov, 1971; Watson, 1972), consequently resulting in cellular defects, aging and death (Harley et al., 1990; Hayflick, 1979; Lundblad and Szostak, 1989). Besides, double-stranded DNA breaks (DSBs) are particularly harmful to the cell due to causing chromosome rearrangements. To distinguish DSBs from authentic chromosome ends is another challenge that an organism must face (Dewar and Lydall, 2012). To solve these critical problems cells evolved variable mechanisms including a special chromosome end nucleoprotein structure named the telomere, which is able to efficiently maintain the stability and integrity of the genome.

\subsubsection{The structure of telomeres}

Telomeres are conserved on their structure throughout the eukaryotic organisms. The structure of a yeast telomere is shown in figure 2.1A. The yeast chromosome ends consist of two subtelomeric regions, $X$ and $Y^{\prime}$ elements, and one telomeric region (reviewed in (Kupiec, 2014; Wellinger and Zakian, 2012)).

$\mathrm{X}$ elements, containing two subregions: Core $\mathrm{X}$ and subtelomeric repeated elements (Louis et al., 1994), are present in almost all chromosome ends with slight differences in size and sequence. $Y^{\prime}$ elements are separated by telomeric repeats from $X$ elements and present in 0-4 tandem repeats (Chan and Tye, 1983). 
The length of the telomeric region varies from yeast ( $c a .350 \mathrm{bp}$ ) to mammals (several $\mathrm{kb}$ ). The yeast telomeric sequence is mainly composed of simple repeats that are usually described as $\mathrm{C}_{1-3} \mathrm{~A} / \mathrm{TG}_{1-3}$. In addition, these sequence repeats are also found at the border between $X$ and $Y^{\prime}$ element, as well as $Y^{\prime}$ and $Y^{\prime}$ elements. However, compared to those in $X$ and $\mathrm{Y}^{\prime}$ elements, the repeats in the telomeric region are more important because deletion of these sequence results in a high genomic instability and the loss of chromosomes (Lundblad and Szostak, 1989; Shampay et al., 1984; Szostak and Blackburn, 1982). This telomeric region can be extended by a reverse transcriptase complex termed telomerase containing an RNA template. Unlike many other organisms, the telomeres of Saccharomyces cerevisiae are irregular and heterogeneous because only a partial RNA template of the telomerase is used for each elongation cycle and diverse short template regions are copied in different elongation rounds (Forstemann and Lingner, 2001). Furthermore, through sequencing it has been shown that the cells from a single colony contain exact identical sequences in the internal half of telomeric region; however, more dynamic combinations are found in the external half, which is therefore thought to be involved in degradation and elongation of the telomeres (Wang and Zakian, 1990). Moreover, a 3'-single strand G-rich tail is present at the very end of the chromosome. These $\mathrm{G}$ tails are usually $12-15$ nucleotides long throughout the cell cycle except during a short period in late S/G1 phase in which it contains 30-200 nucleotides (Larrivee et al., 2004; Wellinger et al., 1993a, b).

\subsubsection{Telomere binding proteins}

There are a number of proteins that bind to the subtelomeric or telomeric region, directly or indirectly, continuously or transiently, functionally or structurally.

The intrinsic components of the telomeres (figure 2.1B) include Rap1 (Repressor Activator Protein), Rif complex (Rif1 and Rif2, Rap1-Interacting Eactor), SIR proteins (Sir2, Sir3 and Sir4,

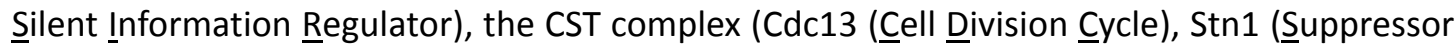
of $\underline{C} \mathrm{dc}$ Thirtee $\underline{\mathrm{N}}$ ) and Ten1 (TElomeric pathways with STN1)) and the Ku heterodimer (Yku70 and Yku80, Yeast Ku protein). 
In wild type cells 15-20 copies of Rap1 bind to each single telomere with the distance of $c a$. 20 base pairs and are the central factors in determining telomere length (Conrad et al., 1990; Gilson et al., 1993; Lustig et al., 1990; Ray and Runge, 1999a, b; Wright and Zakian, 1995). There are key interaction regions for Sir3/4 as well as for Rif1/2 binding present in the C-terminus of Rap1 (Hardy et al., 1992a; Hardy et al., 1992b; Moretti et al., 1994; Wotton and Shore, 1997). Both Rif1 and Rif2 function as negative regulators in telomere elongation (Hardy et al., 1992a; Hardy et al., 1992b; Wotton and Shore, 1997), probably through being involved in the telomere capping, which prevents the access of the double-stranded DNA breaks (DSBs) repair system through covering the telomeres with short telomeric repeats (Ribeyre and Shore, 2012). The SIR complex, composed of Sir2, Sir3 and Sir4, is mainly involved in telomeric silencing by interacting with histones (Gottschling et al., 1990; Pryde and Louis, 1999). Besides, the yeast Ku complex contains two proteins, Yku70 and Yku80, playing the central role in the non-homologous end-joining (NHEJ) machinery and the telomere maintenance (Bonetti et al., 2010a; Bonetti et al., 2010b; Gilson et al., 1993;

\section{A Telomere Components}

DNA elements

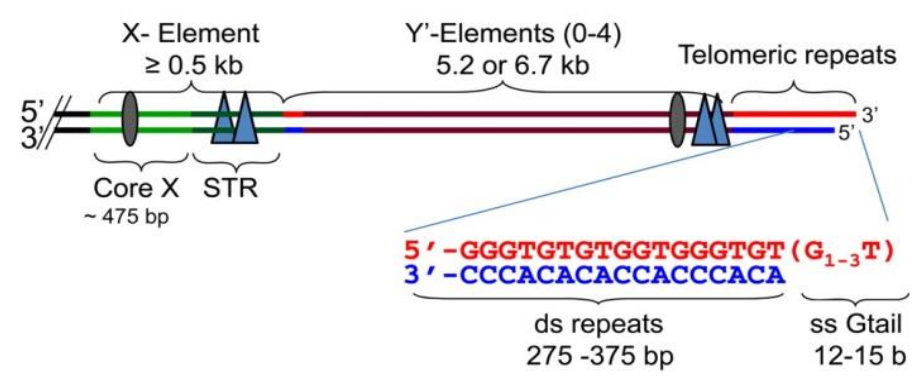

B Main protein components

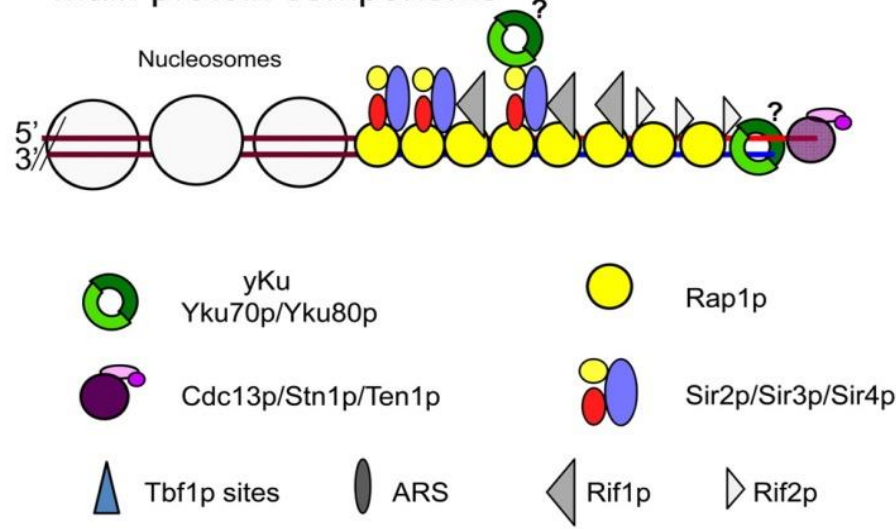

Figure 2.1 Telomeric DNA structure and protein components in S. cerevisiae.

(A) Schematic representation of the subtelomeric $X$ and $Y^{\prime}$ elements as well as the telomeric terminal repeat sequences. Red strand: G-rich strand with $3^{\prime}$ overhanging end. Blue strand: C-rich strand.

(B) Schematic representation of the telomere bound proteins. Open circles represent nucleosomes.

Figure adapted from (Wellinger and Zakian, 2012). 
Palladino et al., 1993; Porter et al., 1996; Vodenicharov and Wellinger, 2007). The Ku complex participates in tethering the telomeres to the perinuclear region (Martin et al., 1999). Furthermore, the Ku complex is able to interact with telomerase RNA (TLC1, IeLomerase Component) and assist the localisation of the telomerase in the nucleus (Gravel et al., 1998; Rathmell and Chu, 1994; Roy et al., 2004; Taccioli et al., 1994). However, the Ku complex might not recruit the telomerase onto the telomeres because of the recent study showing a mutually exclusive recruitment of TLC1 and telomeres by the Ku complex (Pfingsten et al., 2012). Moreover, the other conventional component on telomeres is the CST complex consisting of Cdc13, Stn1 and Ten1. The functions of Stn1 and Ten1 are poorly understood. The core component of the CST complex is Cdc13, which has high affinity and specificity to bind to single-stranded $\mathrm{TG}_{1-3}$ DNA in vitro (Hughes et al., 2000b; Lin and Zakian, 1996; Mitton-Fry et al., 2002; Mitton-Fry et al., 2004; Nugent et al., 1996) and telomeres in vivo (Bourns et al., 1998; Tsukamoto et al., 2001) through its DNA binding domain (Hughes et al., 2000b). Besides, Cdc13 contains a recruitment domain on its $\mathrm{N}$-terminal end, which interacts with Est1 of the telomerase complex and recruits it onto telomeres (Nugent et al., 1996; Pennock et al., 2001; Wu and Zakian, 2011).

In addition, some components are present on telomeres only transiently in given cell cycle phases, e.g. the telomerase complex. As mentioned above, the telomerase is able to be recruited onto the telomeres via an interaction of one of its components, Est1, with Cdc13 in late S phase of the cell cycle (Evans and Lundblad, 1999; Taggart et al., 2002). Furthermore, some DSB recognition factors are also involved in maintaining the telomere length, e.g. Tel1 (TELomere maintenance) and the MRX complex (Mre11 (Meiotic REcombination), Rad50

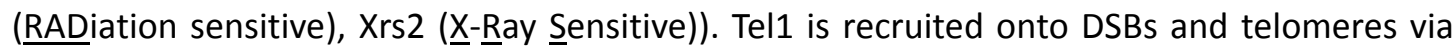
the MRX complex (Nakada et al., 2003; Shima et al., 2005). Lacking of Tel1 or of MRX components leads to short, however stable telomeres (Boulton and Jackson, 1998; Kironmai and Muniyappa, 1997; Tsukamoto et al., 2001). The functions of these factors are proposed to be to participate in the telomerase recruitment (Bianchi and Shore, 2007; Hector et al., 2007; Mantiero et al., 2007; Sabourin et al., 2007). 


\subsubsection{Telomerase}

During DNA replication in eukaryotic cells, DNA polymerases extend short RNA primers composed of 8-12 nucleotides through adding nucleotides to their $33^{2}$-end. DNA polymerases extend one DNA strand in the direction of the growing replication folk (leading strand) and the other strand in a discontinuous fashion which requires many RNA primed Okazaki fragments (lagging strand). The RNA primers subsequently are removed and the gaps are filled and ligated (Olovnikov, 1971; Watson, 1972). However, this leaves two end-replication problems: first, removal of the very start RNA primers results in a shorter newly synthesised product (Watson, 1972); second, although there is an essentiality of 3' single-stranded G-tails at the end of eukaryotic chromosomes, which is important for distinguishing the DSBs and chromosome ends, DNA polymerases generate 3 '-blunt ends on the chromosomes created by the leading strands (Lingner et al., 1995). The telomerase has been evolved to solve the first problem through using its integrated RNA template to elongate the chromosome ends by a reverse transcription.

\subsubsection{Telomerase components}

The telomerase holoenzyme is composed of Est1, Est2, Est3 and the TLC1 RNA (Dandjinou et al., 2004; Hughes et al., 2000a; Zappulla and Cech, 2004). The name EST is an abbreviation of "ever shorter telomeres" from a screen for defective telomere function (Lundblad and Szostak, 1989).

Est1 is a protein predicted with 699 amino acids in length (Lundblad and Szostak, 1989) and has the ability to bind both RNA and $\mathrm{TG}_{1-3}$ single strand DNA that contain $3^{\prime}-\mathrm{OH}$ ends in vitro (DeZwaan and Freeman, 2009; Virta-Pearlman et al., 1996). In its sequence three nuclear localisation signals have been identified that might interact with importin alpha, Srp1 (Hawkins and Friedman, 2014). The expression level of EST1 is cell cycle regulated: it is low in the telomerase inactive $\mathrm{G} 1$ phase (ca. 20 molecules/cell) and high in the telomerase active late S/G2 phase (ca. 110 molecules/cell) (Taggart et al., 2002; Wu and Zakian, 2011). 
Although the telomerase activity is independent of Est1 in vitro (Cohn and Blackburn, 1995), Est1 directly binds to a TLC1 stem loop (Seto et al., 2002) and contributes to the telomeric localisation of the telomerase (Chan et al., 2008) as well as to the telomerase-telomere recruitment via direct interaction with Cdc13 in vivo (Evans and Lundblad, 1999; Qi and Zakian, 2000; Wu and Zakian, 2011). In addition, Est1 is predicted to activate telomerase through recruiting Est3 onto the complex (Tuzon et al., 2011).

Est2 confers the activity of the reverse transcriptase to the telomerase (Lingner et al., 1997). Est2 is composed of 884 amino acids and contains a long basic $\mathrm{N}$-terminal region that includes three conserved aspartate residues essential for its activity (Friedman and Cech, 1999; Lingner et al., 1997). In addition, the N-terminus of Est2 bears the ability to interact with TLC1 and Est3 (Friedman and Cech, 1999; Friedman et al., 2003; Talley et al., 2011). The expression level of EST2 is quite low ( $<40$ molecules/cell) and $T L C 1$ dependent (only $c a .50 \%$ in $t / c 1 \Delta$ strain) (Taggart et al., 2002).

Unlike Est1 and Est2, synthesis of the functional Est3 protein needs a programmed translational frameshift (Morris and Lundblad, 1997). The functional, full-length Est3 is a protein with 181 amino acids; a truncated Est3 of 93 amino acids can be formed without the frameshift and its function has not been found yet (Morris and Lundblad, 1997). The full length Est2 interacts with Est1 directly and associates with the telomerase in an Est1 dependent manner (Osterhage et al., 2006; Tuzon et al., 2011). Est3 has also the ability to bind to the long basic N-terminus of Est2 (Friedman et al., 2003; Talley et al., 2011) and to telomeres in G1 phase with yet unknown functions (Tuzon et al., 2011).

TLC1 (IeLomerase Component) is a low abundant RNA (ca. 30 molecules/cell) longer than 1000 nucleotides; TLC1 is utilised as a template for reverse transcription and a scaffold for the formation of the telomerase complex (Mozdy and Cech, 2006; Singer and Gottschling, 1994).

\subsubsection{Telomerase function}

The telomerase carries out the addition of the telomere repeats using its reverse 
transcriptase activity at the end of the S phase of the cell cycle (Raghuraman et al., 2001). The recruitment of the telomerase onto telomeres is shown in figure 2.2. During G1 and early S phase, only an Est2-TLC1 complex is recruited via the interaction between the $\mathrm{Ku}$ complex and a $48 \mathrm{bp}$ stem loop on TLC1; however, this incomplete telomerase is inactive and elimination of the engagement of this complex to telomeres leads to only little telomere shortening (Fisher et al., 2004). Besides, this association is located at $>100 \mathrm{bp}$ from the end of the chromosomes rather than at the very end of the chromosomes (Sabourin et al., 2007). The active telomerase holoenzyme associates with the telomeres in late S/G2 phase of the cell cycle (Chan et al., 2008). The association of this active telomerase with telomeres is

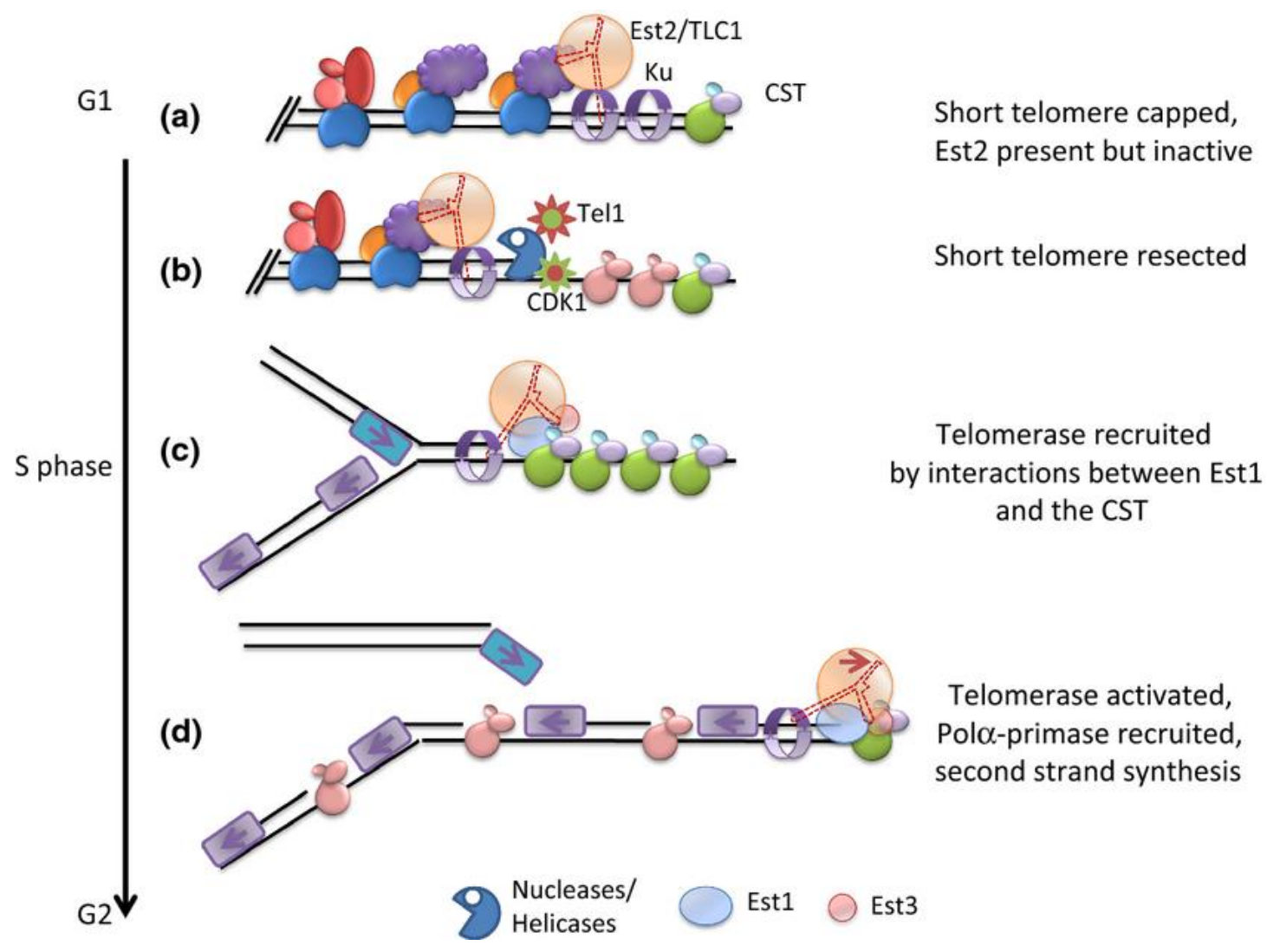

Figure 2.2 Telomere replication. (a) In G1 phase, Est2-TLC1, an incomplete telomerase complex, is inactively present at the telomeres. (b) The chromosome end resection is performed by nucleases and helicases, which are activated by CDK1 and Tel1, to create single strand DNA platforms for association of the CST complex. (c) The telomerase holoenzyme is loaded via interactions between Est1 and the CST complex. (d) The active telomerase holoenzyme elongates the G-rich strand and the CST complex recruits the DNA polymerase alpha-primase, a subunit of the DNA polymerase alpha, to complete the DNA replication.

Figure adapted from (Kupiec, 2014). 
related to the interaction between Est1 and Cdc13. In late S/G2 phase the telomeric binding level of Cdc13 is dramatically increased for facilitating an enhanced recruitment of the telomerase holoenzyme onto the telomeres (Chan et al., 2008; Taggart et al., 2002). Upon association, the telomerase uses short stretches within the sequence 5'-CACACACCCACACCAC- $3^{\prime}$ in TLC1 as templates to elongate telomeres in a heterogeneous fashion (Forstemann and Lingner, 2001; Lin et al., 2004).

\subsubsection{TLC1 and the telomerase life cycle}

Around $90 \%$ of $T L C 1$ is non-polyadenylated and consists of 1157 nucleotides (poly(A)- TLC1);

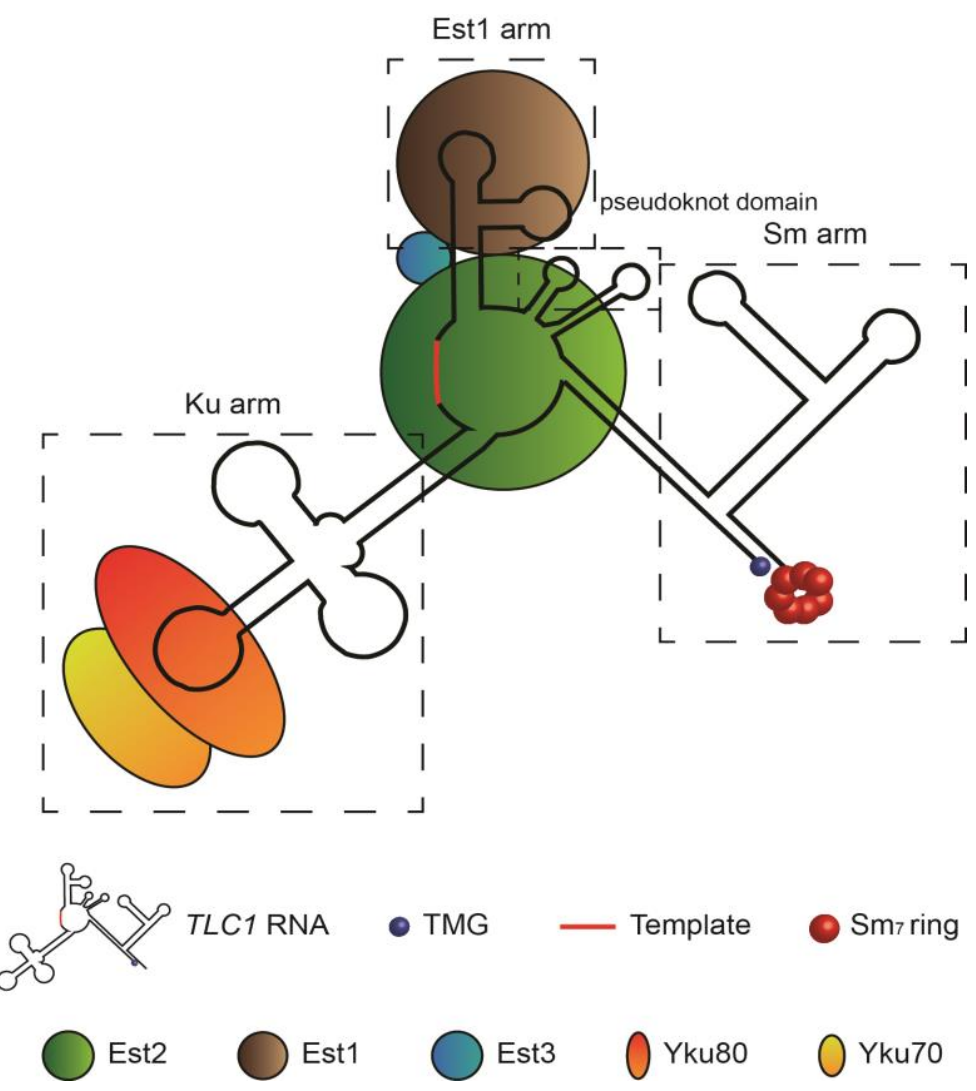

Figure 2.3 TLC1 is a scaffold in constructing the telomerase. Yku80, Est2 and Est1 bind to TLC1. Est3 is involved in telomerase formation through interaction with Est2. However, Est3 is also able to interact with Est1. The $\mathrm{Sm}_{7}$ ring binds to the 3'-end of $T L C 1$ important for its processing and stabilization. Besides, TLC1 contains a TMG Cap structure, which is common in RNA polymerase II produced non-coding RNA, and a core sequence (red part of TLC1), which is utilised as the template for reverse transcription. 
however, $5-10 \%$ of $T L C 1$ exists in longer polyadenylated forms that differ in the length of the $3^{\prime}$-parts and polyadenylation tails (poly(A) ${ }^{+}$TLC1) (Bosoy et al., 2003; Chapon et al., 1997; Noel et al., 2012). Only the form of poly(A) ${ }^{-}$TLC1 is incorporated in the mature telomerase (Bosoy et al., 2003).

The active poly(A) $T L C 1$ is the scaffold for composition of the telomerase (figure 2.3). In addition to the core region, which includes the template for reverse transcription, TLC1 contains a domain for association of the reverse transcriptase Est2 and a conserved pseudoknot domain with yet unknown function, which is however usually thought to be important for maintaining the secondary structure (Dandjinou et al., 2004; Lin et al., 2004; Livengood et al., 2002; Qiao and Cech, 2008; Zappulla and Cech, 2004). Furthermore, TLC1 possesses three other duplex arms, termed Est1 arm, Ku arm and Sm arm. The Est1 arm is the interaction region of Est1, which is essential for the telomerase activity in vivo (Seto et al., 2002). The Ku complex binds to the $\mathrm{Ku}$ arm and this interaction is essential for nuclear localisation of the telomerase and its telomeric recruitment in the $\mathrm{G} 1$ phase of the cell cycle (Fisher et al., 2004; Gallardo et al., 2008; Stellwagen et al., 2003; Vega et al., 2007). Nevertheless, compared to the telomerase RNA in ciliates (ca. 160 nucleotides) or mammalian cells (ca. 450 nucleotides), the size of the TLC1 of Saccharomyces cerevisiae is much larger (Singer and Gottschling, 1994) and an artificial 384-nucleotide TLC1 lacking most non-protein-binding regions is still able to maintain stable telomeres in vivo (Zappulla et al., 2005).

The life cycle of $T L C 1$ and the telomerase is demonstrated in figure 2.4. TLC1 shares many features with some classes of small nuclear RNAs (snRNAs), which are involved in mRNA splicing. Similar to other RNA polymerase II products, e.g. mRNAs, snRNAs, etc., TLC1 is initially capped with 7-monomethyl guanosine $\left(\mathrm{m}^{7} \mathrm{G}\right.$ ) and tailed with poly-adenylates (Abou Elela and Ares, 1998; Chapon et al., 1997; Seipelt et al., 1999). However, like some classes of snRNAs but unlike mRNAs, the poly-adenylated TLC1 receives a seven-Sm protein (Smb1, Smd1, Smd2, Smd3, Sme1, Smx2 and Smx3, abbr. $\mathrm{Sm}_{7}$ ) ring that binds near its $3^{\prime}$ end and this association triggers two subsequent events: $5^{\prime}$ hypermethylation and $3^{\prime}$ degradation (Seto et al., 1999). In budding yeast, the hypermethylation converts the $m^{7} G$ cap into a 
2,2,7-trimethylguanosin (TMG) cap in the nucleolus through the methyltransferase Tgs1 (TrimethylGuanosine Synthase), which is also involved in the cap hypermethylation of snRNAs and small nucleolar RNAs (snoRNAs) (Franke et al., 2008). It must be noted that in higher eukaryotes snRNAs shuttle to the cytoplasm to acquire the $\mathrm{Sm}_{7}$ ring and to be hypermethylated due to the cytoplasmic localisation of the Sm proteins and Tgs1 and subsequently these modifications promote the nuclear re-import of snRNAs (reviewed in (Matera et al., 2007)). Also similar to snRNAs, in the $3^{\prime}$ end region of poly(A) ${ }^{+}$TLC1 a Nab3 (Nuclear polyAdenylated RNA-Binding) and an Nrd1 (Nuclear pre-mRNA ㅁown-regulation) binding site have been identified (Noel et al., 2012) indicating that TLC1 undergoes 3' modification through the Nrd1-Nab3-Sen1 pathway, which is one of the pathways that mediates RNA polymerase II transcription termination (Steinmetz et al., 2001; Vasiljeva et al., 2008). This pathway is thought to trigger the nuclear RNA exosome to remove the poly $(A)^{+}$ tail of TLC1 under the protection of the $\mathrm{Sm}_{7}$ complex (Coy et al., 2013; Noel et al., 2012). TLC1 undergoes a nucleo-cytoplasmic shuttling supposed to be important for maturation of the telomerase complex (Ferrezuelo et al., 2002; Gallardo et al., 2008). Similar to the snRNA transport in metazoan (reviewed in (Hopper, 2006)), the nuclear export of TLC1 has been identified to be mediated by the Crm1/Xpo1 pathway (Gallardo et al., 2008), which mainly uses the Ran/GTPase dependent exportin Xpo1 (EXPOrtin)/Crm1 (hㅏromosome Region Maintenance) to transport large macromolecules from the nucleus to cytoplasm (Neville et al., 1997; Stade et al., 1997). TLC1 cytoplasmic presence is thought to be important for the telomerase formation, where $T L C 1$ assembles with the Est protein components (reviewed in (Gallardo and Chartrand, 2008)). After assembly of the Est proteins in the cytoplasm, TLC1 is

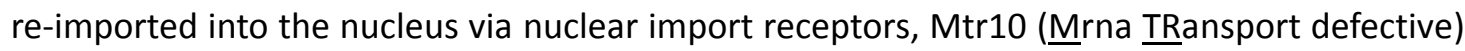
and Pdr6/Kap122 (KAryoPherin) (Ferrezuelo et al., 2002; Gallardo et al., 2008). Interestingly, recent data show an unexpected Mtr10-independent nuclear import pathway for Est1, which is mediated by the importin alpha, Srp1 (Suppressor of $\underline{\mathrm{RPb}} 1$ ), and these data suggest an alternative nuclear import pathway for telomerase components (Hawkins and Friedman, 2014). 


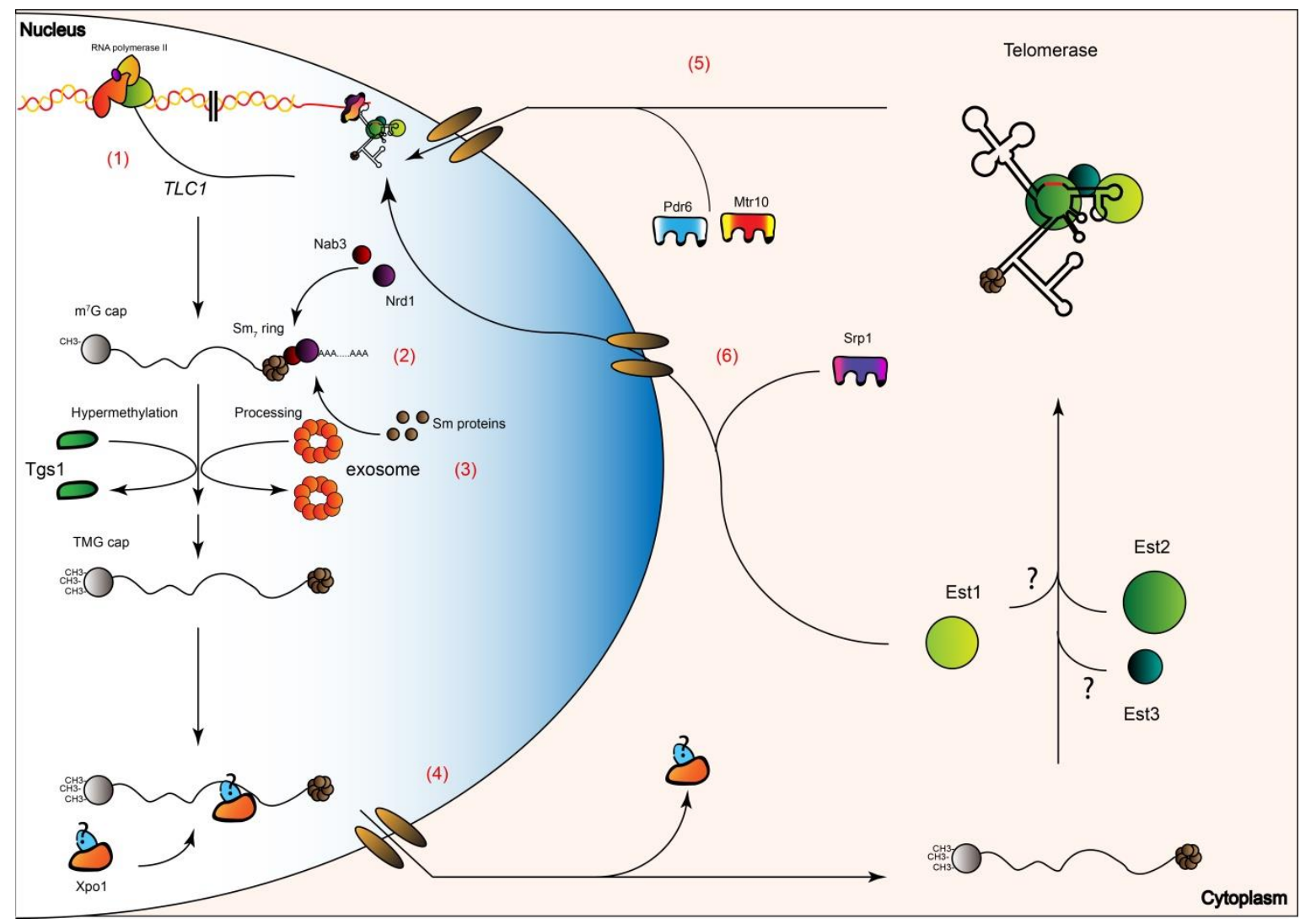

Figure 2.4 The life cycle of TLC1 and the telomerase. (1) TLC1 is synthesised by RNA polymerase II. (2) Immature $T L C 1$ contains a poly $(\mathrm{A})^{+}$tail and $\mathrm{m}^{7} \mathrm{G}$ cap. On its $3^{\prime}$ part its $\mathrm{Sm}$, Nab3 and Nrd1 binding sites are recognised by the $\mathrm{Sm}_{7}$ ring complex, $\mathrm{Nab3}$ and $\mathrm{Nrd1}$, respectively. (3) The association of the $\mathrm{Sm}_{7}$ complex and Nab1-Nrd1 transcription termination complex on TLC1 triggers a hypermethylation of the $5^{\prime}-\mathrm{m}^{7} \mathrm{G}$ cap by Tgs1 and a trimming of the $3^{\prime}$-poly $(\mathrm{A})^{+}$tail by the nuclear exosome. (4) Modified TLC1 is exported into the cytoplasm via the $\mathrm{Crm} 1 / \mathrm{Xpo1}$ pathway, which is mediated by the exportin Xpo1/Crm1 with a so far unknown adaptor. (5) In the cytoplasm TLC1 associates with Est proteins and the complex is subsequently re-imported into the nucleus by Mtr10 and Pdr6/Kap122. (6) Est1 is also supposed to be independently re-imported by Srp1. 


\subsection{RNA nuclear processing and quality control}

\subsubsection{RNA processing and export}

\subsubsection{Non-coding RNA processing}

In yeast, the non-coding RNAs transcribed by RNA polymerase II have several classes including, small nuclear RNA (snRNA), small nucleolar RNA (snoRNA), stable unannotated transcripts (SUTs), cryptic unstable transcripts (CUTs), Xrn1 stabilised transcripts (XUTs), telomerase RNA (TLC1), etc. In contrast to the differentiation between non-coding RNA and mRNA in their promoter regions in mammalian cells (de Vegvar et al., 1986; Hernandez and Weiner, 1986; Richard and Manley, 2009), in yeast one of the differentiations between the non-coding RNA produced by RNA polymerase $I$ and mRNA is in the way of how transcription termination takes place. Predominantly, snRNA, snoRNA, CUTS and TLC1 use an Nrd-Nab3-Sen1 pathway (also referred to NRD pathway) to mediate their termination and processing (Arigo et al., 2006; Noel et al., 2012; Steinmetz et al., 2001). Although only partially understood, these RNA are co-transcriptionally protected and stabilised by recruitment of the specific protein components: H/ACA or C/D-box proteins for snoRNA and Sm family proteins for snRNA and TLC1. Besides, the multi-heterodimer Nrd1 (Nuclear

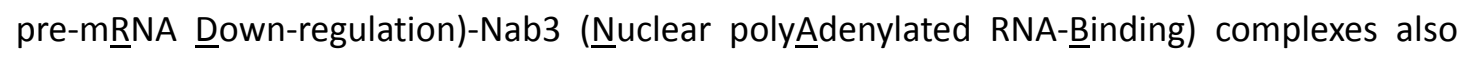
bind to their recognition sites, the 5'-UCUUG-3' motif for Nab3 and the $5^{\prime}-(U / A) G U A(A / G)-3^{\prime}$ motif for Nrd1 (Carroll et al., 2004; Creamer et al., 2011; Morlando et al., 2002). This binding leads to a shortening of the $5^{\prime}$-end by Lsm2-8 (Kufel et al., 2004) and Rat1 and the 3'-end by the exosome (reviewed in (Slomovic and Schuster, 2011)).

\subsubsection{RNA nuclear export}

The nucleus is separated from the cytoplasm by the nuclear envelope. Nuclear transport occurs through the nuclear pore complex (NPC). RNA is packaged into a large 
ribonucleoprotein complex (RNP) and leaves the nucleus through a series of interactions.

Although in mammalian cells snRNA and snoRNA are exported to the cytoplasm via the CRM1 pathway (Hamm and Mattaj, 1990; Izaurralde et al., 1995; Ohno et al., 2000), whether these non-coding RNA do so in yeast still remains mysterious.

Furthermore, in Saccharomyces cerevisiae, mRNA nuclear export is primarily mediated by the export receptor heterodimer Mex67 (MRNA EXport factor of $67 \mathrm{kDa}$ )-Mtr2 (Mrna IRansport) (Segref et al., 1997), which is able to interact with the phenylalanine-glycine (FG) repeats in the NPC-proteins (Bachi et al., 2000; Grant et al., 2002; Gwizdek et al., 2006; Hobeika et al., 2009; Katahira et al., 1999; Suyama et al., 2000). On its N-terminus, Mex67 possesses an RNP domain and next to it a leucine-rich region, both of which are required for its interactions with RNA and RNA adaptor proteins (Kang and Cullen, 1999; Liker et al., 2000). The proper transcript-protein complexes pass through the NPC and reach its cytoplasmic side. At the cytoplasmic face of the NPC, Rat7 (Ribonucleic Acid Irafficking)/Nup159 (Uclear Pore) docks the ATP-dependent RNA helicase, Rat8/Dbp5 (ㅁead Box Protein), at the NPC (Del Priore et al., 1997; Hodge et al., 1999; Weirich et al., 2004). Both of Rat7/Nup150 and Rat8/Dbp5 are important for dissociation of mRNP factors, e.g. Mex67, from the mRNA and this dissociation is thought to create the directionality for mRNA export (reviewed in (Tieg and Krebber, 2013)). 


\subsubsection{RNA nuclear quality control}

Through investigation of the model organism, Saccharomyces cerevisiae, two complexes have been identified that are involved in the degradation of faulty mRNAs in the nucleus (LaCava et al., 2005; Van Hoof et al., 2000a; Vanacova et al., 2005), the TRAMP complex (LaCava et al., 2005) and the RNA exosome (Mitchell et al., 1997). In addition, these two complexes have been shown to contribute to the maturation of many non-coding RNAs (Allmang et al., 2000; Coy et al., 2013; Van Hoof et al., 2000a).

\subsubsection{The TRAMP complex}

In Saccharomyces cerevisiae, the TRAMP complex is composed of a nuclear $3^{\prime}-5^{\prime}$ RNA

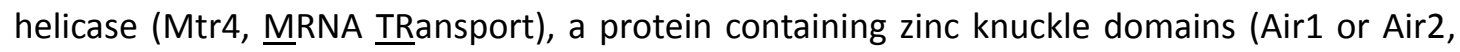

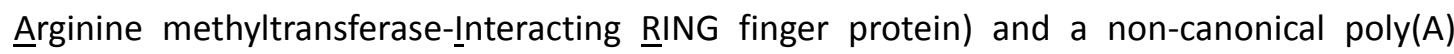
polymerase (Trf4 or Trf5, Iopoisomerase one-Related Function), which gives the name of TRAMP4 or TRAMP5; the TRAMP complexes mark faulty RNAs by adding short poly(A) ${ }^{+}$ sequences on their $3^{\prime}$ tail and this polyadenylation supplies an extended single strand platform to load the nuclear exosome and trigger degradation (Dez et al., 2007; Egecioglu et al., 2006; Houseley and Tollervey, 2006; Kadaba et al., 2004; Kadaba et al., 2006; Paolo et al., 2009; Wyers et al., 2005).

Like the canonical poly(A) polymerase Pap1 (oly(A) Polymerase), Trf4/5 contains a similar catalytic central domain (Vanacova et al., 2005), which allows an addition of 10-50 adenosine residues at the end of RNA that is shorter than the $60-80$ nucleotides poly $(\mathrm{A})^{+}$tail obtained from Pap1 (reviewed in (Eckmann et al., 2011)). Mtr4 is a 3'-5' RNA helicase that consists of an ATPase core of DExH helicase, which is defined by six conserved peptide motifs (de la Cruz et al., 1999), and a unique arch domain similar to some ribosomal proteins (Jackson et al., 2010; LaCava et al., 2005; Weir et al., 2010). In the presence of Mtr4, the polymerase activity of $\operatorname{Trf} 4 / 5$ is suppressed and the poly(A) ${ }^{+}$tail is limited to 3-5 adenosine residues (Jia et al., 2011). Although Trf4/5 marks RNA with this short poly $(A)^{+}$tail, these proteins are lack of the 
ability to associate with RNA, which is however supplied by Air1/2 in the TRAMP complex (Holub et al., 2012; van Hoof et al., 2000b). Air1/2 contains 5 conserved Zinc knuckle motifs, which are involved in both protein-protein and protein-RNA interaction (Fasken et al., 2011). These interactions bridge recognition and polyadenylation on the RNAs and facilitate the degradation of these substrates by the exosome (Fasken et al., 2011; Hamill et al., 2010; Holub et al., 2012). Strikingly, the interaction between Trf4/5 and Mtr4 is Air-independent but requires the helicase core of Mtr4 (Jackson et al., 2010; LaCava et al., 2005; Weir et al., 2010). With this interaction, $\operatorname{Trf} 4 / 5$ is also able to promote the activity of Mtr4 to unwind the highly structured RNA and expose a $3^{\prime}$-ssRNA tail, which can be captured by the exosome (Vanacova et al., 2005).

In addition to RNA degradation, the TRAMP complex has also been proposed to participate in some non-coding RNA transcription termination, e.g. SNR65 and SNR13 (S RNA), by connecting the RNAs to the NRD pathway (Grzechnik and Kufel, 2008; Tudek et al., 2014).

Furthermore, although compositions vary, the differentiation between TRAMP4 and TRAMP5 remains still unclear. Since there are slight differences in their localisations, TRAMP4 and TRAMP5 are proposed to preferentially work on the surveillance of diverse classes of substrates (Fasken et al., 2011; Huh et al., 2003; Paolo et al., 2009).

Finally, some components of the TRAMP complex are able to function apart from the complex, e.g. Mtr4. Its association with the exosome leads to the processing of some non-coding RNA independently of the TRAMP complex (de la Cruz et al., 1998; Kadaba et al., 2006; LaCava et al., 2005; Van Hoof et al., 2000a).

\subsubsection{The nuclear exosome}

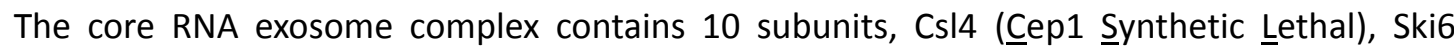
(S uper KIIller), Rrp4, Rrp40, Rrp42, Rrp43, Rrp45, Rrp46 (Ribosomal RNA Processing), Mtr3 and Dis3 (chromosome DISjunction). Six of them (Ski6, Rrp42, Rrp43, Rrp45, Rrp46 and Mtr3) 
form a hexameric ring structure and three of them (CsI4, Rrp4 and Rrp40) constitute a trimeric cap, which is positioned on top of the hexameric ring and together termed Exo-9 (Liu et al., 2006). Exo-9 possesses catalytic activity only if Dis3 is associated with the hexameric ring on the opposite side of the trimeric cap. Exo-9 together with associated Dis3 is named Exo-10 (reviewed in (Das and Das, 2013)). Dis3 is the only catalytic unit in the core RNA exosome and contains both endo- and exo-ribonuclease activity (Mitchell et al., 1997). In the nucleus the core exosome complex associates with Rrp6, Lrp1 (Like RrP6) and Mpp6 (M-Phase Phosphoprotein) to form the nuclear RNA exosome (Synowsky et al., 2009).

The targets of the exosome include a quite wide spectrum of RNAs, comprising both coding and non-coding RNA produced by all three RNA polymerase I, II and III (Gudipati et al., 2012; Schneider et al., 2012). The exosome has been found to degrade most RNA substrates, e.g. mRNA, rRNA, snRNA, snoRNA, tRNA and CUT, etc., and this degradation occurs always together with an oligoadenylation (Schneider et al., 2012). Especially in the nuclear mRNA quality control, the nuclear exosome (Exo-10 with Rrp6, Lrp1 and Mpp6) participates in removing aberrant mRNAs, together with additional factors, e.g. Rat1, a nuclear 5'-3' exoribonuclease (Bousquet-Antonelli et al., 2000; Burkard and Butler, 2000; Libri et al., 2002; Torchet et al., 2002; Zenklusen et al., 2002). 


\section{Materials and methods}

\subsection{Materials}

\subsubsection{Chemical and consumables}

All chemicals, solutions and consumables in this thesis were obtained from the following companies if not stated otherwise:

AppliChem (Munich/Germany), BD Biosciences (Heidelberg/Germany), Carl Roth (Karlsruhe/ Germany), GE Healthcase (Freiburg/Germany), Invitrogen (Frankfurt am Main/Germany), Macherey-Nagel (Dueren/Germany), Merck (Darmstadt/Germany), New England Biolabs (Frankfurt am Main/Germany), OMNILAB GmbH (Bremen/Germany), Promega (Mannheim/ Germany), Peqlab (Erlangen/Germany), Roche Diagnostics (Mannheim/Germany), Sarstedt (Nuernbrecht/Germany), Serva Feinbiochemika (Heidelberg/Germany), Sigma-Aldrich (Munich/Germany), Thermo Fisher Scientific (Schwerte/Germany), Th. Geyer (Renningen/ Germany), VWR International (Darmstadt/ Germany)

\begin{tabular}{|l|l|}
\hline Chemical, Consumables & Source \\
\hline Agarose NEEO Ultra & Carl Roth, Karlsruhe/Germany \\
\hline Amersham Hybond-N+ Membran & GE Healthcare, Freiburg/Germany \\
\hline Bacto Yeast nitrogen base & Becton Dickinson, Franklin Lakes/USA \\
\hline Cy3-Oligo-dT 50 & Biospring, Frankfurt/Germany \\
\hline DAPI & Merck, Darmstadt/Germany \\
\hline Deionised Formamide & AppliChem, Munich/Germany \\
\hline DIG RNA labeling mix, 10x & Roche Diagnostics, Mannheim/Germany \\
\hline dNTPs & Thermo Fisher Scientific, Schwerte/Germany \\
\hline 5-Fluoroorotic Acid (5-FOA) & ApolloScientific, Stockport/UK \\
\hline Formaldehyde 37\% (ACS reagent) & Sigma-Aldrich, Taufkirchen/Germany \\
\hline Galactose & Acros Organics, Geel/Belgium \\
\hline GFP-Trap_A & ChromoTek, Martinsried/Germany \\
\hline Glass beads 0.2-0.5 mm & Carl Roth, Karlsruhe/Germany \\
\hline IgG-Sepharose ${ }^{\text {TM }}$ beads & GE Healthcare, Freiburg/Germany \\
\hline Nitrocellulose Membran (Protran) & PerkinElmer, Waltham/USA \\
\hline Poly-L-Lysine & Sigma-Aldrich, Taufkirchen/Germany \\
\hline cOmplete Protease Inhibitor cocktail & Roche Diagnostics, Mannheim/Germany \\
\hline Protease inhibitor cocktail for yeast & Sigma-Aldrich, Taufkirchen/Germany \\
\hline Protein G Sepharose & Applied Biosystems, Foster City/USA \\
\hline Raffinose & Serva, Heidelberg/Germany \\
\hline Rotiphorese Gel 30 & Carl Roth, Karlsruhe/Germany \\
\hline Sucrose & Carl Roth, Karlsruhe/Germany \\
\hline & 18 \\
\hline
\end{tabular}




\begin{tabular}{|l|l|}
\hline Salmon sperm DNA & Sigma-Aldrich, Taufkrichen/Germany \\
\hline Sorbitol & Carl Roth, Karlsruhe/Germany \\
\hline 12-well microscope slide & Thermo Fisher Scientific, Schwerte/Germany \\
\hline CSPD & Roche Diagnostics, Mannheim/Germany \\
\hline Hoechst 33342 & Sigma-Aldrich, Taufkirchen/Germany \\
\hline IgG Sepharose & GE Healthcare, Freiburg/Germany \\
\hline tRNA & Sigma-Aldrich, Taufkirchen/Germany \\
\hline Yeast extract & Carl Roth, Karlsruhe/Germany \\
\hline Ribonucleoside vanadyl complexes & Sigma-Aldrich, Taufkirchen/Germany \\
\hline Fujifilm Super RX & Fujifilm, Tokyo/Japan \\
\hline & \\
\hline Kits & Source \\
\hline ECL Prime Western Blotting Detection Kit & GE Healthcare, Freiburg/Germany \\
\hline DIG-High Prime DNA Labeling and Detection & Roche Diagnostics, Mannheim/Germany \\
Starter Kit II & \\
\hline NucleoBond PC 100 & Macherey-Nagel, Dueren/Germany \\
\hline NucleoSpin Plasmid & Macherey-Nagel, Dueren/Germany \\
\hline peqGOLD Gel Extraction kit & Peqlab, Erlangen/Germany \\
\hline GoTaq qPCR Master Mix & Promega, Mannheim/Germany \\
\hline pGEM-T vector system & Promega, Mannheim/Germany \\
\hline & \\
\hline Size Standards & Source \\
\hline Lambda DNA/EcoRI+HindIII DNA Ladder & Thermo Fisher Scientific, Schwerte/Germany \\
\hline GeneRuler 1 kb DNA Ladder & Thermo Fisher Scientific, Schwerte/Germany \\
\hline PageRuler Prestained Protein Ladder & Thermo Fisher Scientific, Schwerte/Germany \\
\hline PageRuler Unstained Protein Ladder & Thermo Fisher Scientific, Schwerte/Germany \\
\hline
\end{tabular}




\subsubsection{Enzymes and antibodies}

All enzymes were used with the appropriate buffers according to the protocols of the manufactures.

To be used in western blot (WB) analyses all antibodies were diluted in 1-2\% milk powder/TBST. To be used in immunofluorescence (IF) or fluorescence in situ hybridization (FISH) experiments all antibodies were diluted in antibody blocking buffer (5-10\% heat inactivated fetal bovine serum/PBST). To be used in southern blot (SB) analyses the antibody was diluted in $1 \times$ Blocking reagent (1\% blocking reagent in $1 \times$ Maleic acid buffer)

\begin{tabular}{|l|l|l|}
\hline Enzymes & Source \\
\hline DreamTaq DNA Polymerase & Thermo Fisher Scientific, Schwerte/Germany \\
\hline FastAP Alkaline Phosphatase & Thermo Fisher Scientific, Schwerte/Germany \\
\hline Phusion High-Fidelity DNA Polymerase & New England Biolabs, Frankfurt /Germany \\
\hline KAPAHiFi Polymerase & Peqlab, Erlangen/Germany \\
\hline Restriction enzymes & $\begin{array}{l}\text { Thermo Fisher Scientific, Schwerte/Germany } \\
\text { New England Biolabs, Frankfurt /Germany }\end{array}$ \\
\hline RiboLock Rnase Inhibitor & Thermo Fisher Scientific, Schwerte/Germany \\
\hline RNase A & AppliChem, Munich/Germany \\
\hline T4 DNA Ligase & Thermo Fisher Scientific, Schwerte/Germany \\
\hline T7 RNA Polymerase & Thermo Fisher Scientific, Schwerte/Germany \\
\hline Zymolase & Seikagaku Corporation, Tokyo/Japan \\
\hline & \multicolumn{2}{|l|}{} \\
\hline Antibodies & Dilution & Source \\
\hline Anti-mouse-HRP (goat) & $1: 5000-1: 10000$ (WB) & Dianova, Hamburg/Germany \\
\hline Anti-rabbit-HRP (goat) & $1: 10000-1: 20000$ (WB) & Dianova, Hamburg/Germany \\
\hline Anti-mouse AlexaFluor 488 (sheep) & $1: 1000$ (IF) & Invitrogen, Frankfurt/Germany \\
\hline Anti-rabbit AlexaFluor 488 (sheep) & $1: 1000$ (IF) & Invitrogen, Frankfurt/Germany \\
\hline Anti-GFP (mouse) & $1: 250$ (IF), 1:1000 (WB) & Santa Cruz, Heidelberg/Germany \\
\hline Anti-GFP (rabbit) & $1: 250$ (IF), 1:1000 (WB) & Santa Cruz, Heidelberg/Germany \\
\hline Anti-myc (mouse) & $1: 250$ (IF), 1:1000 (WB) & Santa Cruz, Heidelberg/Germany \\
\hline Anti-myc (rabbit) & $1: 250$ (IF), 1:1000 (WB) & Santa Cruz, Heidelberg/Germany \\
\hline Anti-Nop1 (rabbit) & $1: 5000$ (IF and WB) & \\
\hline Anti-Hem15 (rabbit) & $1: 5000$ (WB) & Gift from Roland Lill, Marburg \\
\hline Anti-Zwf1 (rabbit) & $1: 2500$ (WB) & Gift from Roland Lill, Marburg \\
\hline Anti-Mtr4 (rabbit) & $1: 1000$ (WB) & $\begin{array}{l}\text { Roche Diagnostics, Mannheim/ } \\
\text { Germany }\end{array}$ \\
\hline Anti-Digoxigenin-FITC & $1: 200$ (FISH) & $\begin{array}{l}\text { Roche Diagnostics, Mannheim/ } \\
\text { Germany }\end{array}$ \\
\hline Anti-Digoxigenin-AP & $1: 10000$ (NB) \\
\hline & \multicolumn{2}{|l|}{ (W) } \\
\hline
\end{tabular}




\subsubsection{Instruments and software}

\begin{tabular}{|c|c|}
\hline Instrument & Company \\
\hline Fusion FX7 & Peqlab, Erlangen/Germany \\
\hline FastPrep-24 & MP Biomedicals, Illkirch/France \\
\hline Leitz Biomed Typ 020-507-010 & Leica, Wetzlar/Germany \\
\hline Leica DMI6000B & Leica, Wetzlar/Germany \\
\hline Leica DFC360FX & Leica, Wetzlar/Germany \\
\hline Hamamatsu 1394 ORCA-ERA camera & Leica, Wetzlar/Germany \\
\hline Rotor Gene Q & Qiagen, Hilden/Germany \\
\hline Nikon Eclipse E400 & Nikon, Duesseldorf/Germany \\
\hline Heraeus Pico 21 centrifuge & Thermo Fisher Scientific, Schwerte/Germany \\
\hline Heraeus Fresco 21 centrifuge & Thermo Fisher Scientific, Schwerte/Germany \\
\hline Heraeus Multifuge X3R centrifuge & Thermo Fisher Scientific, Schwerte/Germany \\
\hline Heraeus B6060 & Heraeus, Hanau/Germany \\
\hline Heraeus B6420 & Heraeus, Hanau/Germany \\
\hline Optimax X-Ray Film Processor & PROTEC, Oberstenfeld/Germany \\
\hline Vacuum Blot & ITF, Marl/Germany \\
\hline Cross Linker Bio Link BLX 365 & Peqlab, Erlangen/Germany \\
\hline Perfect Blue Semi dry Electroblotter & Peqlab, Erlangen/Germany \\
\hline Gilson Pipetman P2/P10/P100/P1000 & Gilson, Inc., Middleton/USA \\
\hline Intelli Scan 1600 & Quato Technology, Braunschweig/Germany \\
\hline INTAS UV-system & Intas, Göttingen/Germany \\
\hline MyCycler Thermal Cycler & BioRad, Müchen/Germany \\
\hline NanoDrop2000 & Thermo Fisher Scientific, Schwerte/Germany \\
\hline Milli-Q water purification & Millipore, Eschborn/Germany \\
\hline Software & Source \\
\hline Bio 1D & Peqlab, Erlangen/Germany \\
\hline Image J & http://imagej.net/ \\
\hline Leica LAS AF & Leica, Wetzlar/Deutschland \\
\hline SilverFast v3.1.1 & LaserSoft Imaging AG, Kiel/Germany \\
\hline Adobe Creative Suite Design Standard & Adobe, San Jose/USA \\
\hline Microsoft office & Microsoft, Redmond/USA \\
\hline ApE editor & biologylabs.utah.edu/jorgensen/wayned/ape/ \\
\hline FileMaker & FileMaker, Inc. \\
\hline
\end{tabular}




\subsubsection{Strains, plasmids and oligonucleotides}

\subsubsection{Strains}

\begin{tabular}{|c|c|c|}
\hline \multicolumn{3}{|c|}{ E.coli strain } \\
\hline Name & Genotype & Source \\
\hline $\mathrm{DH} 5 \alpha$ & fhuA2 lac(del)U169 phoA glnV44 Ф80' lacZ(del)M15 gyrA96 recA1 relA1 endA1 thi-1 hsdR17 & Krebber lab \\
\hline \multicolumn{3}{|c|}{ Yeast strains } \\
\hline Number & Genotype & Source \\
\hline HKY36 & Mat $\alpha$ ura3-52 leu2 11 his3 200 & (Winston et al., 1995) \\
\hline HKY46 & Mata mtr10-1 ura3-52 lys2-301 ade2 & (Liu et al., 1999) \\
\hline HKY82 & Mat $\alpha$ mtr10::HIS3 ura3 leu2 trp his3 ade2 + pURA-MTR10 & (Senger et al., 1998) \\
\hline HKY97 & Mato mtr10::HIS3 ura3 leu2 trp his3 ade2 + pURA-MTR10 & Krebber lab \\
\hline HKY124 & Mata rat7-1 ura3-52 leu2 $\Delta 1$ his $3 \Delta 200$ & (Gorsch et al., 1995) \\
\hline HKY128 & 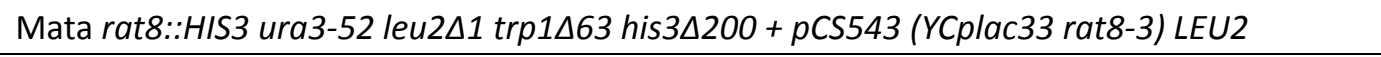 & (Snay-Hodge et al., 1998) \\
\hline HKY130 & Mata rat8-2 ura3-52 leu2 $\Delta 1 \operatorname{trp} 1 \Delta 63$ & (Snay-Hodge et al., 1998) \\
\hline HKY145 & Mat $\alpha$ XPO1-GFP-TRP1 ura3 leu2 his3 & Krebber lab \\
\hline HKY206 & Mat $\alpha$ xpo1::LEU2 ade2-1 his leu trp1-1 ura3-1 ade2-1 + pCEN TRP1 xpo1-1 & (Taura et al., 1998) \\
\hline HKY209 & Mata pdr6::HIS3 lys2 trp1 ura3-52 leu2 $\Delta 1$ & Krebber lab \\
\hline HKY280 & Mata PAB1-GFP-KAN leu2 trp1 ura3-52 pep4-3 pre1-407 prb1-1122 & (Zenklusen et al., 2001) \\
\hline HKY305 & Mata prp4-1 ura1 trp1 his7 ade1 ade2 & Krebber lab \\
\hline HKY306 & 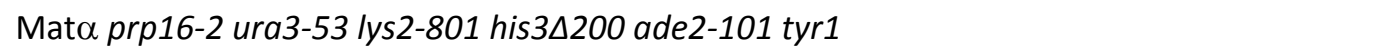 & Krebber lab \\
\hline HKY316 & Mata MTR10-9xMYC-TRP1 ura3-52 leu2 $\Delta 1 \operatorname{trp} 1 \Delta 63$ & Krebber lab \\
\hline
\end{tabular}




\begin{tabular}{|c|c|c|}
\hline HKY380 & 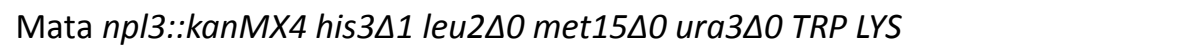 & Euroscarf \\
\hline HKY381 & Mat $\alpha$ his $3 \Delta 1$ leu $2 \Delta 0$ ura $3 \Delta 0$ lys $2 \Delta 0$ & Euroscarf \\
\hline HKY382 & Mat $\alpha$ rat8::HIS3 ura3-52 leu2 $21 \operatorname{trp} 1 \Delta 63+p R A T 8-M Y C ~ 2 \mu$ LEU2 & Krebber lab \\
\hline HKY428 & Mat $\alpha$ mtr4-G677D ura3-52 leu2 $\Delta 1$ his3 $\triangle 200+p C E N-g b p 2-S 15 A$ URA3 & Krebber lab \\
\hline HKY446 & Mat $\alpha$ sup45-2 ura3-1 ade2-1 his5-2 can1-100 & (Stansfield et al., 1997) \\
\hline HKY578 & Mat $\alpha$ TIF4631-3xMYC-HIS3 ura leu trp his ade & (Knop et al., 1999) \\
\hline HKY644 & Mato mex67::HIS3 ade2 his3 leu2 trp1 ura3 + pUN100-mex67-5 LEU2 CEN & (Segref et al., 1997) \\
\hline HKY648 & Mat $\alpha$ mex67::HIS3 ade2 his3 leu2 trp1 ura3 + pUN100-MEX67-GFP LEU2 CEN & (Segref et al., 1997) \\
\hline HKY661 & Mat $\alpha$ mex67::HIS3 ade2 his3 leu2 trp1 ura3 + pUN100-mex67-5 LEU2 CEN & Krebber lab \\
\hline HKY948 & 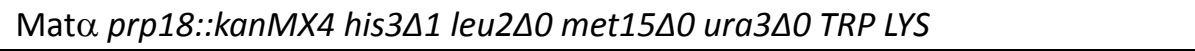 & Euroscarf \\
\hline HKY1008 & 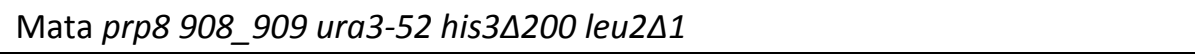 & Krebber lab \\
\hline HKY1028 & Mat $\alpha$ rrp6::kanMX4 his3 $\Delta 1$ leu2 $\Delta 0$ met $15 \Delta 0$ ura3 $\Delta 0$ TRP LYS & Euroscarf \\
\hline HKY1072 & 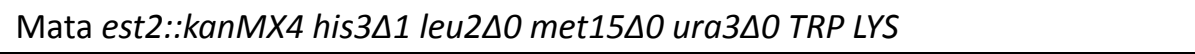 & Euroscarf \\
\hline HKY1073 & 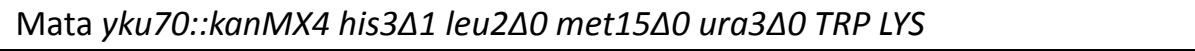 & Euroscarf \\
\hline HKY1074 & 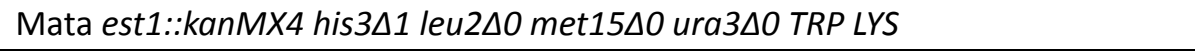 & Euroscarf \\
\hline HKY1075 & 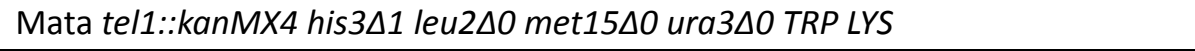 & Euroscarf \\
\hline HKY1076 & 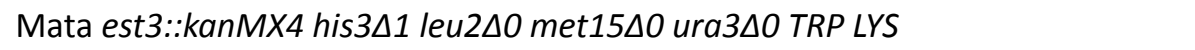 & Euroscarf \\
\hline HKY1077 & 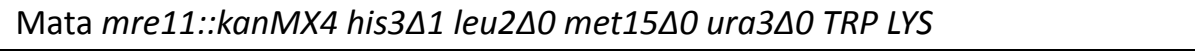 & Euroscarf \\
\hline HKY1078 & 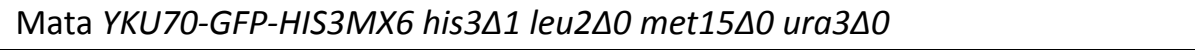 & (Huh et al., 2003) \\
\hline HKY1079 & Mata RAP1-GFP-HIS3MX6 his3 $\Delta 1$ leu2 $\Delta 0$ met $15 \Delta 0$ ura3 $\Delta 0$ & (Huh et al., 2003) \\
\hline HKY1081 & Diploid TLC1/tIc1::LEU2 RAD52/rad52::TRP ura3-1 leu2-3 his3-11 trp1-1 ade2-1 & (Gallardo et al., 2008) \\
\hline HKY1082 & Mata RAP1-13xMYC-HIS3 ura3-1 leu2-3 his3-11 trp1-1 ade2-1 & (Gallardo et al., 2008) \\
\hline HKY1094 & 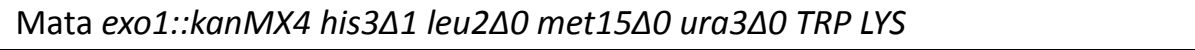 & Euroscarf \\
\hline HKY1111 & 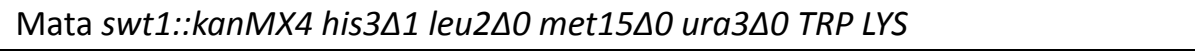 & Euroscarf \\
\hline HKY1112 & 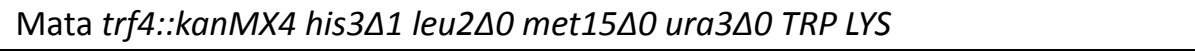 & Euroscarf \\
\hline HKY1136 & Mata RRP6-GFP-HIS3MX6 his $3 \Delta 1$ leu $2 \Delta 0$ met $15 \Delta 0$ ura $3 \Delta 0$ & (Huh et al., 2003) \\
\hline HKY1171 & Mata TRF4-GFP-HIS3MX6 his3 $\Delta 1$ leu2 $\Delta 0$ met $15 \Delta 0$ ura3 $\Delta 0$ & (Huh et al., 2003) \\
\hline
\end{tabular}




\begin{tabular}{|c|c|c|}
\hline HKY1172 & Mata RRP44-GFP-HIS3MX6 his3 $\Delta 1$ leu2 $\Delta 0$ met $15 \Delta 0$ ura3 $\Delta 0$ & (Huh et al., 2003) \\
\hline HKY1236 & 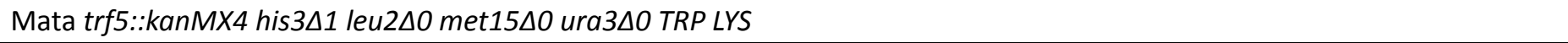 & Euroscarf \\
\hline HKY1237 & 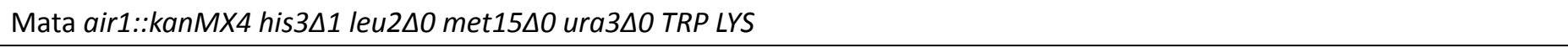 & Euroscarf \\
\hline HKY1238 & 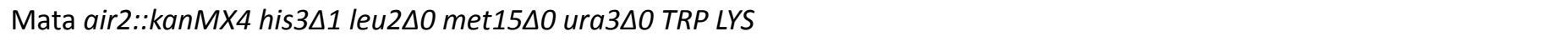 & Euroscarf \\
\hline HKY1240 & 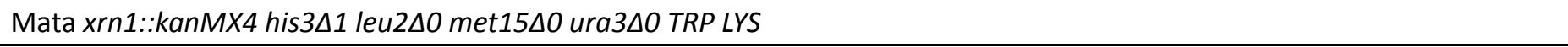 & Euroscarf \\
\hline HKY1242 & Mata DBP5-GFP-HIS3MX6 his3 $\Delta 1$ leu $2 \Delta 0$ met $15 \Delta 0$ ura3 $\Delta 0$ & (Huh et al., 2003) \\
\hline HKY1266 & Mata MEX67-GFP-HIS3MX6 his3 $\Delta 1$ leu2 $\Delta 0$ met $15 \Delta 0$ ura3 $\Delta 0$ & (Huh et al., 2003) \\
\hline HKY1267 & Mata MTR4-GFP-HIS3MX6 his3 $\Delta 1$ leu2 $\Delta 0$ met $15 \Delta 0$ ura $3 \Delta 0$ & (Huh et al., 2003) \\
\hline HKY1290 & Mata $p G A L-3 \times H A-R R P 44-H I S 3 M X 6$ his $3 \Delta 1$ leu $2 \Delta 0$ met15 $\Delta 0$ ura3 $\Delta 0$ & (Schneider et al., 2009) \\
\hline HKY1291 & 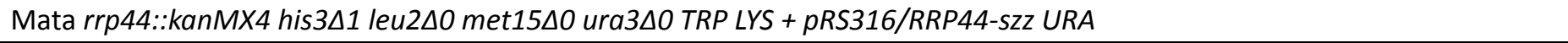 & (Schneider et al., 2009) \\
\hline HKY1292 & Mata EST1-6xGLY-12xMYC 3xFLAG-12xMYC-6xGLY-Est2 leu2 trp1 ura3-52 prb- prc- pep4-3 & (Lubin et al., 2012) \\
\hline HKY1293 & Mat $\alpha$ t/c1::HIS ura3-52 lys2-801 trp- $\triangle 1$ his3- $\triangle 200$ leu2- $\triangle 1+p C E N$ URA3 TLC1 & (Lubin et al., 2012) \\
\hline HKY1294 & Mata EST1-6xGLY-12xMYC 3xFLAG-12xMYC-6xGLY-Est2 t/c1::HIS leu2 trp1 ura3-52 prb- prc ${ }^{-}$pep4-3 + pCEN URA3 TLC1 & (Lubin et al., 2012) \\
\hline HKY1302 & Mata EST1-6xGLY-12xMYC 3xFLAG-12xMYC-6xGLY-Est2 xpo1::LEU2 leu2 trp1 ura3-52 prb- prc- pep4-3 + pCEN TRP xpo1-1 & This study \\
\hline HKY1304 & 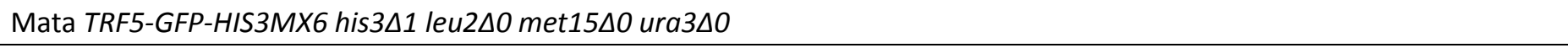 & (Huh et al., 2003) \\
\hline HKY1332 & Mata EST1-6xGLY-12xMYC 3xFLAG-12xMYC-6xGLY-Est2 rat7-1 leu2 trp1 ura3-52 prb- prc- pep4-3 & This study \\
\hline HKY1334 & Mata EST1-6xGLY-12xMYC 3xFLAG-12xMYC-6xGLY-Est2 rat8-2 leu2 trp1 ura3-52 prb- prc ${ }^{-}$pep4-3 & This study \\
\hline HKY1336 & Mata EST1-6xGLY-12xMYC 3xFLAG-12xMYC-6xGLY-Est2 mex67::HIS leu2 trp1 ura3-52 + pUN100-mex67-5 LEU2 CEN & This study \\
\hline HKY1353 & Mata mex67::HIS3 xpo1::TRP1 ura + pUN100-mex67-5 LEU2 CEN + pxpo1-1::HIS3 & (Brune et al., 2005) \\
\hline HKY1377 & 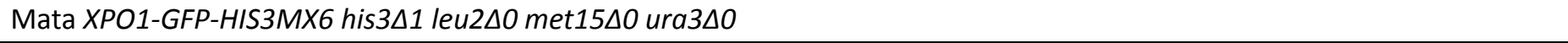 & (Huh et al., 2003) \\
\hline HKY1396 & Mat $\alpha$ rat8-2 yku70::kanMX4 ura leu trp & This study \\
\hline HKY1397 & Mat $\alpha$ mex67::HIS3 yku70::kanMX4 ura leu trp + pUN100-mex67-5 LEU2 CEN & This study \\
\hline HKY1398 & Mat $\alpha$ rat7-1 yku70::kanMX4 ura leu trp & This study \\
\hline HKY1399 & 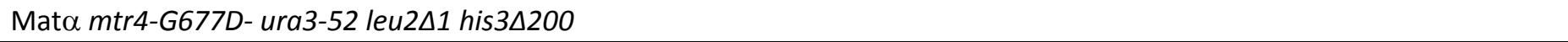 & Krebber lab \\
\hline HKY1444 & Mat $\alpha$ xpo1::LEU2 yku70::KanMX4 ade2-1 his leu trp1-1 ura3-1 + pCEN TRP1 xpo1-1 & This study \\
\hline HKY1445 & Mata mex67::HIS3 xpo1::TRP1 yku70::KanMX4 ura + pUN100-mex67-5 LEU2 CEN + pxpo1-1::HIS3 & This study \\
\hline HKY1463 & Mat $\alpha$ EST1-3xMYC-kanMX4 his3 $\Delta 1$ leu2 $\Delta 0$ ura3 $\Delta 0$ lys $2 \Delta 0$ & This study \\
\hline
\end{tabular}

\section{4}




\begin{tabular}{|c|c|c|}
\hline HKY1464 & Mat $\alpha$ xpo1::LEU2 EST1-3xMYC-kanMX4 ade2-1 his leu trp1-1 ura3-1 + pCEN TRP1 xpo1-1 & This study \\
\hline HKY1465 & Mat $\alpha$ mex67::HIS3 EST1-3xMYC-kanMX4 ade2 his3 leu2 trp1 ura3 + pUN100-mex67-5 LEU2 CEN & This study \\
\hline HKY1466 & Mata mex67::HIS3 xpo1::TRP1 EST1-3xMYC-kanMX4 ura + pUN100-mex67-5 LEU2 CEN + pxpo1-1::HIS3 & This study \\
\hline HKY1467 & Mat EST2-3xMYC-kanMX4 his3 $\Delta 1$ leu2 $\Delta 0$ ura3 $\Delta 0$ lys $2 \Delta 0$ & This study \\
\hline HKY1468 & Mat $\alpha$ xpo1::LEU2 EST2-3xMYC-kanMX4 ade2-1 his leu trp1-1 ura3-1 + pCEN TRP1 xpo1-1 & This study \\
\hline HKY1469 & Mat $\alpha$ mex67::HIS3 EST2-3xMYC-kanMX4 ade2 his3 leu2 trp1 ura3 + pUN100-mex67-5 LEU2 CEN & This study \\
\hline HKY1470 & Mata mex67::HIS3 xpo1::TRP1 EST2-3xMYC-kanMX4 ura + pUN100-mex67-5 LEU2 CEN + pxpo1-1::HIS3 & This study \\
\hline HKY1471 & t/c1::LEU2 rad52::TRP EST2-3XMYC-KanMX4 ura3-1 leu2-3 his3-11 trp1-1 ade2-1 & This study \\
\hline HKY1472 & t/c1::LEU2 rad52::TRP EST1-3XMYC-KanMX4 ura3-1 leu2-3 his3-11 trp1-1 ade2-1 & This study \\
\hline
\end{tabular}




\subsubsection{Plasmids}

\begin{tabular}{|c|c|c|}
\hline Number & Construct & Source \\
\hline pHK12 & CEN URA3 pADH-NLS-NES-GFP-GFP & Krebber lab \\
\hline pHK20 & CEN LEU2 pUN100-MEX67-GFP & (Segref et al., 1997) \\
\hline pHK40 & CEN HIS3 XPO1 & Krebber lab \\
\hline pHK43 & CEN URA3 XPO1-GFP & Krebber lab \\
\hline pHK85 & CEN pRS313-HIS3 & (Sikorski and Hieter, 1989) \\
\hline pHK86 & CEN pRS314-TRP1 & (Sikorski and Hieter, 1989) \\
\hline pHK87 & CEN pRS315-LEU2 & (Sikorski and Hieter, 1989) \\
\hline pHK88 & CEN pRS316-URA3 & (Sikorski and Hieter, 1989) \\
\hline pHK260 & $2 \mu$ LEU2 RAT8-MYC & (Snay-Hodge et al., 1998) \\
\hline pHK413 & LEU2 ProtA-TEV-MTR10 & (Senger et al., 1998) \\
\hline pHK453 & CEN TRP1 mtr10-7 & (Senger et al., 1998) \\
\hline pHK475 & $2 \mu$ URA3 $p$ GAL1-GBP2-S13,15,17A-GFP & Krebber lab \\
\hline pHK491 & 3XMYC-kanMX6 & (Knop et al., 1999) \\
\hline pHK492 & $3 \times M Y C-H I S 3 M X 6$ & (Knop et al., 1999) \\
\hline pHK636 & $2 \mu$ LEU2 pGAL1-MYC-RAT8 & Krebber lab \\
\hline pHK637 & $2 \mu$ TRP1 RAT8 & Krebber lab \\
\hline pHK643 & CBP-TEV-ProtA K.I.TRP1 & (Puig et al., 2001) \\
\hline pHK644 & CBP-TEV-ProtA K.I.URA3 & (Puig et al., 2001) \\
\hline pHK645 & pGal1-ProtA-TEV-CBP K.I.TRP1 & (Puig et al., 2001) \\
\hline pHK648 & $2 \mu$ TRP1 pGAL1-RAT8-MYC & Krebber lab \\
\hline pHK649 & $2 \mu$ URA3 pGAL1-RAT8-MYC & Krebber lab \\
\hline pHK670 & 9xMYC kanMX4 & (Gauss et al., 2005) \\
\hline pHK1238 & $2 \mu$ URA3 TRF4-2xMYC & (Fasken et al., 2011) \\
\hline
\end{tabular}




\begin{tabular}{|c|c|c|}
\hline pHK1239 & $2 \mu$ URA3 TRF5-2xMYC & (Fasken et al., 2011) \\
\hline pHK1240 & $2 \mu$ URA3 MTR4-2xMYC & (Fasken et al., 2011) \\
\hline pHK1336 & $2 \mu$ URA3 NLS-TRF4-2xMYC & This study \\
\hline pHK1337 & $2 \mu$ URA3 NLS-MTR4-2xMYC & This study \\
\hline pHK1351 & URA3 RRP44-SZZ & (Schneider et al., 2009) \\
\hline pHK1352 & LEU2 RRP44-SZZ & (Schneider et al., 2009) \\
\hline pHK1353 & LEU2 RRP44-exo & (Schneider et al., 2009) \\
\hline pHK1354 & LEU2 RRP44-endo & (Schneider et al., 2009) \\
\hline pHK1355 & LEU2 RRP44-exo-endo & (Schneider et al., 2009) \\
\hline pHK1411 & URA3 NLS-RRP44-SZZ & Krebber lab \\
\hline pHK1412 & LEU2 NLS-RRP44-SZZ & Krebber lab \\
\hline
\end{tabular}




\subsubsection{Oligonucleotides}

The gene sequences are depicted in upper case letters, other regions, e.g. restriction sites, transcription sites, are indicated in lower case letters

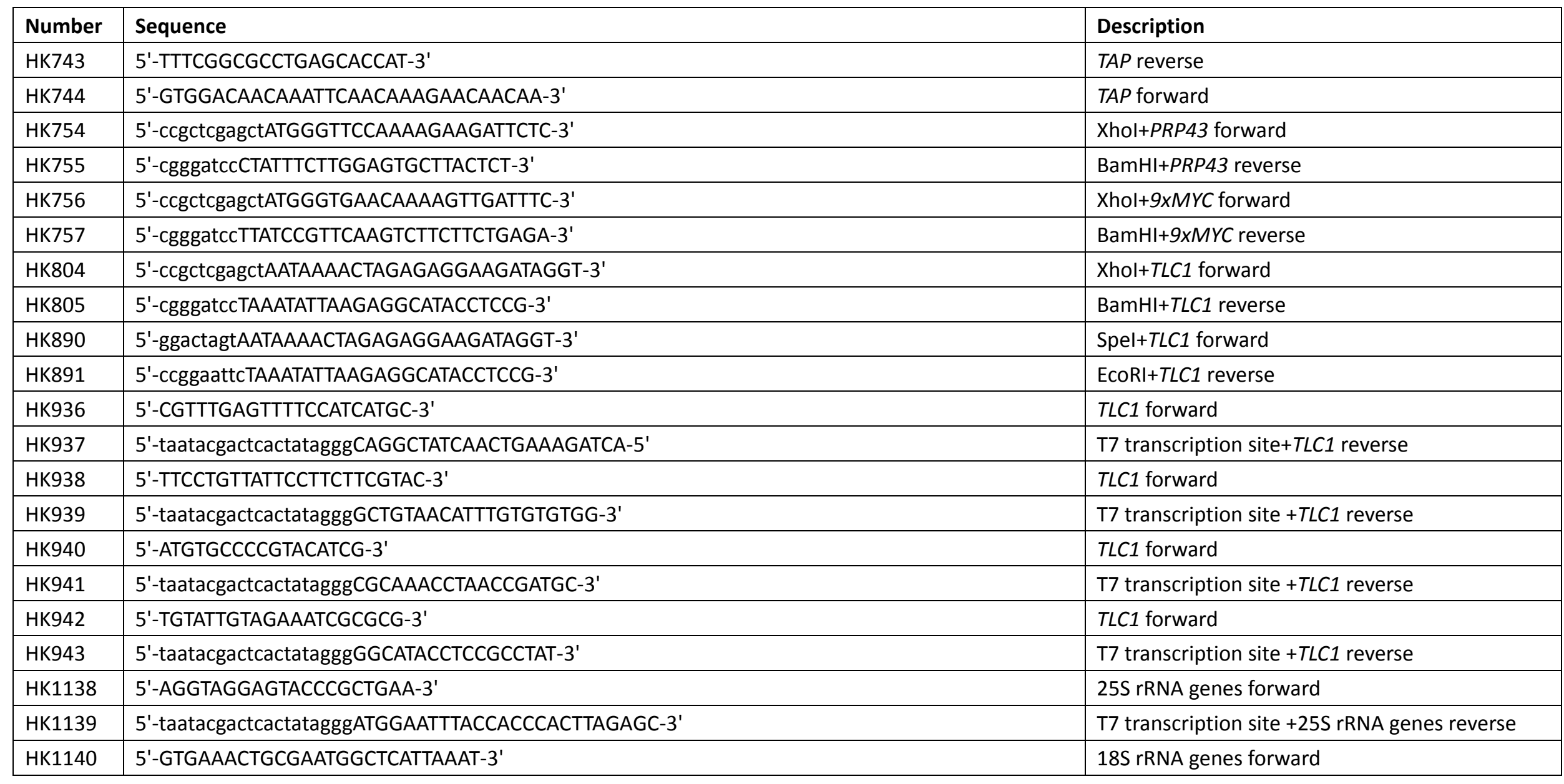




\begin{tabular}{|c|c|c|}
\hline HK1141 & 5'-taatacgactcactatagggAATCGAACCCTTATTCCCCGTTA-3' & T7 transcription site $+18 \mathrm{~S}$ rRNA genes reverse \\
\hline HK1142 & 5'-AAACTTTCAACAACGGATCTCTTGG-3' & 5.8S rRNA genes forward \\
\hline HK1143 & 5'-taatacgactcactatagggAAATGACGCTCAAACAGGCATG-3' & $\mathrm{T} 7$ transcription site $+5.8 \mathrm{~S}$ rRNA genes reverse \\
\hline HK1379 & 5'-CTTGATGTATATTTTTTTGTATTGTA-3' & TLC1 forward \\
\hline HK1380 & 5'-taatacgactcactatagggCAATTAAAAGCGCTTATAAAG-3' & T7 transcription site $+T L C 1$ reverse \\
\hline HK1396 & 5'-CATGGCCGTTCTTAGTTGGTGG-3' & 18S rRNA genes forward \\
\hline HK1397 & 5'-ATTGCCTCAAACTTCCATCGGC-3' & 18S rRNA genes reverse \\
\hline HK1404 & 5'-TCGCGAAGTAACCCTTCGTG-3' & SNR6 forward \\
\hline HK1405 & 5'-AAACGGTTCATCCTTATGCAGG-3' & SNR6 reverse \\
\hline HK1467 & 5'-ccaaaaaagaaaagaaaagttGATTCTACTGATCTGTTCGATGTTTTC-3' & $N L S+M T R 4$ forward \\
\hline HK1468 & 5'-aacttttcttttcttttttggCATCCTTCGTATATAATCTATATTTCTTGCAG-3' & NLS +MTR4 reverse \\
\hline HK1469 & 5'-ccaaaaaagaaaagaaaagttGGGGCAAAGAGTGTAACAGC-3' & NLS +TRF4 forward \\
\hline HK1470 & 5'-aacttttcttttcttttttggCATATTTCAAGTATAGTTCCCTTGCTTATTCA-3' & $N L S+T R F 4$ reverse \\
\hline HK1483 & 5'-ATGCCAAAAAAGAAAAGAAAAGTT-3' & NLS forward \\
\hline HK1492 & 5'-taataggactcactatagggAAATAAATCTCTTTGTAAAACGGTTCATCC-3' & T7 transcription site $+S N R 6$ reverse \\
\hline HK1517 & 5'-ccaaaaaagaaaagaaaagttTCAGTTCCCGCTATCGCC-3' & $N L S$ sequence+DIS3 forward \\
\hline HK1518 & 5'-aacttttcttttcttttttggCATGTTGTTTTGGCCTGTATGATG-3' & $N L S$ sequence $+D I S 3$ reverse \\
\hline HK1539 & 5'-DIG-CCACCACACACACCCACACCC-3' & DIG labelled Telomere probe \\
\hline HK1742 & 5'-TGATTTGTTAAGTGACTCTAAGCCTGATTTTAAAACGGGAATATTATG-3' & YKU70 forward \\
\hline HK1743 & 5'-AAATATTGTATGTAACGTTATAGATATGAAGGATTTCAATCGTCTTTA-3' & YKU7O reverse \\
\hline HK1761 & 5'-Сy3-GCGCACACACAAGCATCTACACTGACACCAGCATACTCGAAATTCTTTGG-Сy3-3' & Cy3 labelled TLC1 probe \\
\hline HK1787 & 5'-Cy5-CAATTAAAAGCGCTTATAAAGCGATATACAAGTAC-Cy5-3' & Cy5 labelled TLC1 probe \\
\hline HK1788 & 5'-Cy5-CGCGCGATTTCTACAATACAAAAAATATACATCAAG-Cy5-3' & Cy5 labelled TLC1 probe \\
\hline HK1789 & 5'-Cy3-CGATAAGATAGACATAAAGTGACAGCGCTTAGCACCGTCTGTTTGC-Сy3-3' & Cy3 labelled TLC1 probe \\
\hline Hk1790 & 5'-Су3-СCTACTCGTATTTTTTCTCTGTCACATCGTTCGATGTACGGGGCACATTTGG-Cy3-5' & Cy3 labelled TLC1 probe \\
\hline HK1830 & 5'-CAATTGATGCTGATGAGGACATCACCGTCCAAGTGCCAGATACTCCTACTcgtacgctgcaggtcgac-3' & pHK491+EST1 forward \\
\hline HK1831 & 5'-TAATATATTTCATATTATGATTTTTTTCCCTCACCATTACTTGTTCTCTCAatcgatgaattcgagctcg-3' & pHK491+EST1 reverse \\
\hline
\end{tabular}

\section{9}


\begin{tabular}{l|l} 
HK1845 & 5'-AAATTCAACACTTGCAAGCATATATATATATATATATACATATAGTTAATcgtacgctgcaggtcgac-3' \\
\hline
\end{tabular}

HK1846
5'-TTCCTTATCAGCATCATAAGCTGTCAGTATTTCATGTATTATTAGTACTAatcgatgaattcgagctcg-3'
pHK491+EST2 forward

pHK491+EST2 reverse 


\subsection{Methods}

\subsubsection{General methods}

\subsubsection{Generation of strains, plasmids and oligonucleotides}

All S. cerevisiae and E. coli strains, plasmids, oligonucleotides used in this study are listed in the material part (see section 3.1.4).

The plasmids pHK1336 and pHK1337 were created directly through PCR reactions, with which the gene sequence of the nuclear localisation signal (NLS) was incorporated into the plasmids with the specific primers that contain the NLS sequence. In the PCR reactions for generating pHK1336, pHK1238 was used as the template and HK1469 and HK1470 were used as the primer pair. In the PCR reactions for generating pHK1337, pHK1240 was used as the template and HK1467 and HK1468 were used as the primer pair. A routine PCR reaction was described in Polymerase chain reaction (PCR) (section 3.2.3.1). Subsequently, $1 \mu$ I DnI $(10 \mathrm{u} / \mu \mathrm{l}$, Thermo Fisher Scientific) was directly added to the PCR reaction mixture and the mixture was incubated at $37^{\circ} \mathrm{C}$ for 1 hour and then used for E. coli transformation (see Transformation, section 3.2.4.1). Dpnl recognized and digested $5^{\prime}-G^{M-} A^{\wedge} T C-3^{\prime}$ sites, which existed only in the template plasmids that were isolated from $\mathrm{DH} 5 \alpha$ strain. Therefore, after transformation, only the transformed cells containing newly synthesized plasmids were able to grow on the selective plates.

The yeast strains were generated by strains crossing or transformation. Strain crossing was described in Yeast sporulation and tetrad analysis (section 3.2.1.4). Homologous recombination was achieved through Transformation (section 3.2.4.1) of cells with 1ng PCR products (e.g. KanMX4, $3 \times M Y C$ ) that contained a homologous sequence of approximate 50 nucleotides in length of the target genes on each side.

The yeast strains HKY1302, HKY1332, HKY1334, HKY1336 were generated by crossing HKY206+HKY1292, HKY124+HKY1292, HKY582+HKY1292, HKY661+HKY1292, respectively. The haploid cells showing both temperature sensitivities (mutations) and expression of 
EST1-MYC and EST2-MYC (detection see SDS-polyacrylamide gel electrophoresis (SDS-PAGE) and western blot, section 3.2.2.4) were selected.

The yeast strains HKY1396, HK1397, HK1398 were generated by crossing HKY582+HKY1073, HKY644+HKY1073, HKY124+HKY1073, respectively. The haploid cells showing both temperature sensitivities (mutations) and geneticin $(200 \mu \mathrm{g} / \mathrm{ml}$ used in medium or plate) resistance (KanMX4 marker) were selected.

The yeast strains HKY1444 and HKY1445 were created by transformation of HKY206 and HKY1353, respectively, with the PCR products that were generated from the gDNA of HKY1073 with the primer pair HK1742+HK1743. Through homologous recombination the YKU7O gene was replaced by the KanMX4 gene. Desired YKU7O knock-out strains showing geneticin resistance were selected.

The yeast strains HKY1463, HKY1464, HKY1465, HKY1466, HKY1472 were created through transforming HKY381, HKY206, HKY644, HKY1353, HKY1081 respectively, with the PCR products that were generated from the plasmid pHK491 with the primer pair HK1830+HK1831. Through homologous recombination the 3XMYC:KanMX4 sequence was incorporated upstream of the stop codon of the EST1 gene. Desired MYC-tagged EST1 strains that showed geneticin resistance were selected.

The yeast strains HKY1467, HKY1468, HKY1469, HKY1470, HKY1471 were created through transforming HKY381, HKY206, HKY644, HKY1353, HKY 1081 respectively, with the PCR products that were generated from the plasmid pHK491 with the primer pair HK1845+HK1846. Through homologous recombination the 3XMYC:KanMX4 sequence was incorporated upstream of the stop codon of EST2 gene. Desired MYC-tagged EST2 strains that showed geneticin resistance were selected.

\subsubsection{Media and plates}

All media were autoclaved before usage. Heat sensitive materials (antibiotics, some carbon sources and 5'-FOA, etc.) were filter-sterilised before being added to the autoclaved media. 
Solid agar plates were made by adding $1.5 \%$ agar (for E. coli) or $1.8 \%$ agar (for yeast) to the autoclaved corresponding media.

\subsubsection{Cell cultivation}

Cells were grown in YPD, YP or selective media. YP medium is a full medium without a carbon source to which the carbon source (glucose, raffinose or galactose, etc.) is added separately. The selective media lack one or several amino acids or nucleobases, which allow the growth of the yeast strains that contain select markers in plasmids or in the genomes. Yeast cells were cultivated in the medium at $25^{\circ} \mathrm{C}$ and the cells were harvested at the middle-logarithmic phase (cell concentration: $1 \times 10^{7}$ to $3 \times 10^{7}$ cells $/ \mathrm{ml}$ ) if not indicated otherwise. Yeast cells were harvested through centrifugation at $2000-4000 \times \mathrm{g}$ and $4^{\circ} \mathrm{C}$ (Heraeus Multifuge X3R centrifuge) for 10 minutes (the cell culture volume $>=5 \mathrm{ml}$ ) or centrifugation at $10000-20000 \times g$ and $4^{\circ} \mathrm{C}$ (Heraeus Fresco 21 centrifuge) or room temperature (Heraeus Pico 21 centrifuge) for 1 minute (the cell culture volume<5ml).

\section{YPD medium:}

$1 \%(w / v)$ Yeast extract

$2 \%(w / v)$ Peptone

$2 \%(w / v)$ Glucose

\section{YP medium:}

$1 \%(\mathrm{w} / \mathrm{v})$ Yeast extract

$2 \%(w / v)$ Yeast extract

$1 \%(w / v)$ Peptone

Selective media:

$2 \%(w / v)$ Drop-out mix 
$0.17 \%(w / v)$ Nitrogen base

$0.51 \%(w / v)$ Ammonium sulfate

$2 \%(w / v)$ Glucose

Additional desired amino acids/bases

\section{Drop-out mix (-Ura -Leu -His -Lys -Ade -Trp):}

$2 \mathrm{~g} / \mathrm{l}$ each of the following components: Alanine, Arginine, Asparagine, Aspartic acid, Cysteine, Glutamine, Glutamic acid, Glycine, Inositol, Isoleucine, Methionine, Phenylalanine, Proline, Serine, Threonine, Tyrosine, Valine.

$0.2 \mathrm{~g} / \mathrm{l}$ Para-aminobenzoic acid

The following components were selectively added as desired:

$0.5 \mathrm{~g} / \mathrm{l}$ Adenine

2g/l Histidine

$10 \mathrm{~g} / \mathrm{l}$ Leucine

$2 \mathrm{~g} / \mathrm{l} \mathrm{Lysine}$

2g/l Tryptophan

$2 \mathrm{~g} / \mathrm{I}$ Uracil

\section{B-plates:}

$0.17 \%(w / v)$ Nitrogen base

$0.51 \%(w / v)$ Ammonium sulfate

$0.3 \%(w / v)$ Agar

$2 \%(w / v)$ Glucose

FOA plates (5'-Fluoro-oratic-acid):

$0.17 \%(w / v)$ Nitrogen base

$0.51 \%(w / v)$ Ammonium sulfate

$0.2 \%(w / v)$ Drop-out mix (all amino acids and bases included)

$0.3 \%(w / v)$ Agar 
$2 \%(w / v)$ Glucose

$0.1 \%(w / v)$ FOA

LB medium is a standard nutrient-rich medium used for cultivating $E$. coli cells. $E$. coli cells were grown overnight at $37^{\circ} \mathrm{C}$ in $\mathrm{LB}$ medium with or without antibiotics. The addition of antibiotics allows the growth of the $E$. coli cells with the specific antibiotic resistances from the plasmids. E. coli cells were harvested through centrifugation at $4000 \times \mathrm{g}$ (Heraeus Fresco 21 centrifuge) for 10 minutes (cell culture volume $>=5 \mathrm{ml}$ ) or at $10000-20000 \times \mathrm{g}$ and room temperature (Heraeus Pico 21 centrifuge) for 1 minutes (cell culture volume $<5 \mathrm{ml}$ ).

\section{LB medium (pH7.5):}

$1 \%(w / v)$ Tryptone

$0.5 \%(w / v)$ Yeast extract

$0.5 \%(w / v) ~ N a C l$

Antibiotics:

As desired, one or some of the following antibiotics are added:

$100 \mu \mathrm{g} / \mathrm{ml}$ Ampicillin

$20 \mu \mathrm{g} / \mathrm{ml}$ Kanamycin

\subsubsection{Yeast sporulation and tetrad analysis}

In Saccharomyces cerevisiae, diploid strains can be created by mating two haploid stains with different mating types (mating type a or $\alpha$ ). Under the condition of nitrogen starvation and poor carbon source the yeast diploid cells undergo meiosis, sporulation and form asci. The genotypes of single spores (tetrads) were analysed through a series of genetic, biochemical and molecular biological methods.

Two haploid strains with different mating types (a or $\alpha$ ) were mixed and incubated on YPD 
plates at $25^{\circ} \mathrm{C}$ for $2-3$ days. The diploid cells were selected from the selectable markers (URA3, LEU2, KanMX4, etc.) or the phenotypes (temperature-sensitivity, etc.) from the haploid strains. The diploid strains were cultivated in $2 \mathrm{ml}$ SuperSpo medium for 3 days at $25^{\circ} \mathrm{C}$ untill the asci became visible. The asci were collected by centrifugation at $10000 \times \mathrm{g}$ and room temperature for 1 minute. After washing once in $100 \mu$ sterile $\mathrm{H}_{2} \mathrm{O}$ and centrifugation at $10000 \times \mathrm{g}$ and room temperature for 30 seconds, the cells were resuspended in $50 \mu \mathrm{l}$ P-solution. The cell walls of the spores were digested by adding $2.5 \mu \mathrm{l}$ zymolyase $(10 \mathrm{mg} / \mathrm{ml}$, Sigma) at room temperature and the digestion were monitored under the microscope. After the cell walls were digested (around 5-10 minutes at room temperature), the spores were harvested by centrifugation at $10000 \times \mathrm{g}$ and room temperature for 30 seconds and washed in $100 \mu \mathrm{l}$ P-solution. The spores were again collected through centrifugation at $10000 \times \mathrm{g}$ and room temperature for 30 seconds and finally resuspended in $200 \mu \mathrm{l}$ P-solution. $2.5 \mu \mathrm{l}$ of cells were diluted in $100 \mu \mathrm{l}$ sterile $\mathrm{H}_{2} \mathrm{O}$ and plated on YPD plates. By using the tetrad microscope with a micromanipulator (Nikon Eclipse E400) the four spores from a tetrad were separated. The spores were grown on YPD plates for 2-5 days at $25^{\circ} \mathrm{C}$. The phenotype of the spores were identified through analyses of their select makers (URA3, LEU2, KanMX4, etc.) by incubating them on selective plates, or through verification of their protein tags (myc, GFP, etc.) by western blot, or through identification of their genome content (mutations, truncated genes, knock-out genes, etc.) by PCR, etc.

\section{SuperSpo medium:}

$5 \mathrm{~g} / \mathrm{l}$ Yeast extract

30g/l Potassium acetate

$1 \mathrm{~g} / \mathrm{l}$ Glucose

$80 \mathrm{mg} / \mathrm{l}$ Adenine

$80 \mathrm{mg} / \mathrm{l}$ Uracil

$80 \mathrm{mg} / \mathrm{l}$ Tyrosine

40mg/l Histidine

40mg/l Leucine 
40mg/l Lysine

40mg/l Tryptophan

40mg/l Methionine

40mg/l Arginne

200mg/l Phenylalanine

700mg/l Threonine

\section{$\underline{0.1 \mathrm{M} \text { Potassium phosphate buffer }(\mathrm{pH} 6.5)}$}

$3.3 \%(\mathrm{v} / \mathrm{v}) 1 \mathrm{M} \mathrm{K}_{2} \mathrm{HPO}_{4}$

$6.7 \%(\mathrm{v} / \mathrm{v}) 1 \mathrm{M} \mathrm{KH}_{2} \mathrm{PO}_{4}$

\section{P-solution (pH 6.5):}

0.1M Potassium phosphate buffer pH6.5

1.2M Sorbitol

\subsubsection{Yeast cell lysis}

Cells were collected from $10 \mathrm{ml}-1000 \mathrm{ml}$ cell culture, which was grown to the middle-logarithmic phase if not indicated otherwise, by centrifugation at $4000 \times \mathrm{g}$ and $4^{\circ} \mathrm{C}$ for 10 minute. The cells were washed once with $1 \mathrm{ml}$ sterile $\mathrm{H}_{2} \mathrm{O}$ and transferred to $2 \mathrm{ml}$ screw-cap tubes. The cells were washed once with the ice-cold appropriate buffer (according to applications, e.g. $1 \times$ SDS sample buffer for western blot, PBSKMT for co-immunoprecipitation, RNA co-IP buffer for RNA co-immunoprecipitation). The cells were mixed with the same cell pellet volume of glass beads and double cell pellet volume of the ice-cold buffer. The mixture was vigorously shaken 2-3 times with the Fastprep machine (MP Biomedicals) at a speed of $4.0 \mathrm{~m} / \mathrm{s}$ for 20 seconds. The samples were cooled down on ice between shaking intervals. The cracked cells were finally centrifuged at $20000 \times \mathrm{g}$ for 5 minutes at $4^{\circ} \mathrm{C}$. The supernatants were carefully transferred to fresh $1.5 \mathrm{ml}$ or $2 \mathrm{ml}$ reaction 
tubes and used in following experiments (Western blot analyses, protein precipitation, co-immunoprecipitation or RNA co-immunoprecipiation).

$\underline{1 \times \text { SDS sample buffer: }}$

$33 \%(v / v) 3 \times$ SDS sample buffer

$2.5 \%(v / v) \beta$-Mercaptoethanol

$\underline{3 \times \text { SDS sample buffer: }}$

$0.3 \mathrm{M}$ Tris $\mathrm{pH} 6.8$

$30 \%(v / v)$ Glycerol

$6 \%(w / v) S D S$

$0.01 \%(w / v)$ Brome phenol blue

10×PBS (pH7.4):

$137 \mathrm{mM} \mathrm{NaCl}$

$2.7 \mathrm{mM} \mathrm{KCl}$

$1.8 \mathrm{mM} \mathrm{KH}_{2} \mathrm{PO}_{4}$

$10 \mathrm{mM} \mathrm{Na}{ }_{2} \mathrm{HPO}_{4}$

Adjust $\mathrm{pH}$ to 7.4

\section{PBSKMT:}

$1 \times$ PBS

$3 \mathrm{mM} \mathrm{KCl}$

$2.5 \mathrm{mM} \mathrm{MgCl}{ }_{2}$

0.1-0.5\% (v/v) Triton-X100

Before usage freshly add:

$1 \times$ cOmplete Protease inhibitor cocktail (Roche)

RNA co-IP buffer: 
$150 \mathrm{mM} \mathrm{KCl}$

$25 \mathrm{mM}$ Tris $\mathrm{pH} 7.0$

$2 \mathrm{mM}$ EDTA

$0.1-0.5 \%(v / v)$ Triton-x100

Before usage freshly add:

$1 \times$ cOmplete Protease inhibitor cocktail (Roche)

$1 \mathrm{mM}$ DTT

$0.01 \%(v / v)$ Ribonucleoside vanadyl complexes (Sigma)

\subsubsection{Preparation of microscope slides}

12-well slides (Thermo Fisher Scientific) were used in immunofluorescence or fluorescent in situ hybridization experiments. The wells were incubated with $3 \mu$ poly-L-lysine $(100 \mu \mathrm{g} / \mathrm{ml}$, Sigma) for 5 minutes and were air-dried at $42^{\circ} \mathrm{C}$. Poly-L-lysine is positively charged and is able to enhance the attachment of negatively charged cell wall to the well surface. The wells were washed three times with sterile $\mathrm{H}_{2} \mathrm{O}$ and were air-dried at $42^{\circ} \mathrm{C}$. The slides were stored at $4^{\circ} \mathrm{C}$ for weeks.

\subsubsection{Applications of the microscopes}

The light microscope (Leitz Biomed Typ 020-507-010) was used for counting the cell number and monitoring cell wall digestions (see Yeast sporulation and tetrad analysis, section 3.2.1.4; Nucleo-cytoplasmic fractionation, section 3.2.2.3; Immuno-fluorescence (IF), section 3.2.4.2; RNA fluorescent in situ hybridization (FISH), section 3.2.4.3) .

The tetrad microscope with a micromanipulator (Nikon Eclipse E400) was used to separate single spores from tetrads.

The Fluorescence microscopes (Leica DMI6000B with Leica DFC360FX camera or Hamamatsu 1394 ORCA-ERA camera) were used for capturing and analysing the fluorescent signals in 
immunofluorescence or fluorescent in situ hybridisation experiments. If necessary, deconvolution was applied by using the Leica LAS AF software to acquire images with sharper contrast and higher intensity. In these cases cells were imaged through 6-10 z-stacks $(0.2 \mu \mathrm{m} / \mathrm{stack})$ followed by the blind deconvolution.

\subsubsection{Signal detection, quantification and statistical analyses}

The fluorescent signals from immunofluorescence and fluorescent in situ hybridization experiments were captured by the fluorescence microscopes as described in Applications of the microscopes (section 3.2.1.7). The chemiluminescent signals from western blot and southern blot analyses were detected by the Fusion FX7 system (Peqlab) or Super RX X-ray films (Fujifilm) and Optimax X-Ray Film Processor (PROTEC). Ethidium bromide incorporated nucleic acids were detected by the $312 \mathrm{~nm}$ UV-light with the INTAS UV-system (INTAS).

The intensity of chemiluminescent signals (western blot signals from protein expression analyses or co-immunoprecipitation experiments) was quantified by using the Bio1D software (Peqlab) or ImageJ software (http://imageJ.net). The intensity of fluorescent signals (immunofluorescence or fluorescent in situ hybridization) was quantified by using ImageJ software (http://imageJ.net). The intensity of the fluorescent signals from qRT-PCR experiments was quantified by the Rotor Gene Q system (Qiagen).

In each case of this thesis at least three independent experiments were performed. Western signals were quantified from at least three independent experiments. Fluorescent signals were quantified from 20 to 50 cells. Figures in this thesis represent one experiment. All quantifications are the mean values \pm standard deviations of at least 3 independent experiments. Error bars represent the standard deviations. The mean values and standard deviations were calculated by using Microsoft Excel. The p-values were calculated through paired or unpaired, two tailed t-test by using the Microsoft Excel $(*: 0.01 \leq p \leq 0.05$; $* *$ : $0.001 \leq p<0.01 ; * * *: 0.0001 \leq p<0.001 ; * * * *: 0.00001 \leq p<0.0001)$. 


\subsubsection{Biochemical methods}

\subsubsection{Protein extraction and precipitation}

According to the protocol Yeast lysis (section 3.2.1.5), cells of interest were lysed and centrifuged and the clear supernatants were obtained. The same volume $20 \%(\mathrm{w} / \mathrm{v})$ Trichloroacidic acid (TCA) was added to the supernatants. After vigorously mixing (5-10 seconds), the mixture was incubated on ice for 30 minutes. The mixture was further centrifuged at $20000 \times \mathrm{g}$ and room temperature for 15 minutes. After discarding the supernatant, the pellet was washed twice with $1 \mathrm{ml} 80 \%(\mathrm{v} / \mathrm{v})$ acetone. After centrifugation at $20000 \times \mathrm{g}$ and room temperature for 2 minutes, the supernatant was discarded and the pellet was air-dried. The pellet was dissolved in $1 \times$ SDS sample buffer (recipe in section 3.1.5). The samples were either further used in western blot analyses or stored at $-80^{\circ} \mathrm{C}$

\subsubsection{Co-immunoprecipitation (IP or co-IP)}

Co-immunoprecipitation experiments were used to study interactions among proteins. In these assays proteins of interest were precipitated by using specific antibodies and immobilised beads. The interaction partners of target proteins were co-precipitated and analysed through western blot analyses (SDS-polyacrylamide gel electrophoresis (SDS-PAGE) and western blot, section 3.2.2.4). To precipitate proteinA tagged (TAP, SZZ, etc.) proteins, IgG Sepharose ${ }^{\mathrm{TM}} 6$ Fast Flow (Roche) beads were used. To precipitate GFP tagged proteins, GFP-Trap_A beads (Chromotek), on which GFP antibodies were covalently bound, were used. To precipitate untagged or the other tagged (e.g. myc, HA, etc.) proteins, the appropriate antibodies (e.g. direct antibodies for untagged proteins, anti-myc for myc tagged proteins, etc.) and Protein G Plus agarose beads (Applied Biosystems) were used.

Before co-immunoprecipitation experiments the beads $(10 \mu \mathrm{l}$ IgG Sepharose 6 Fast Flow, $8 \mu \mathrm{l}$ GFP-Trap_A beads or $15 \mu$ l Protein G Plus agarose beads for one sample) were pre-washed with $1 \mathrm{ml}$ ice-cold PBSKMT buffer for 5 times, each time with centrifugation at $500 \times \mathrm{g}$ and $4^{\circ} \mathrm{C}$ 
for 5 minutes. The beads were resuspended in $20 \mu \mathrm{l}$ buffer and stored on ice untill usage.

Cells from $200-500 \mathrm{ml}$ cell cultures (middle-logarithmic phase) were lysed in ice-cold PBSKMT buffer as described in Yeast lysis (section 3.2.1.5). To prevent proteins from digestion, the protease inhibitor cocktail ( $25 \times$, Roche) was added with a final concentration of $1 \times$.

$25-50 \mu \mathrm{l}$ of the clear lysates was mixed with the same volume of the $2 \times$ SDS sample buffer and used in western analyses as input controls. The rest of the clear lysates were incubated with corresponding beads and antibodies at $4^{\circ} \mathrm{C}$ for 3 hours to overnight on a rotator. If necessary, during the incubations $10 \mathrm{mg} / \mathrm{ml}$ RNaseA was added to a final concentration of $50 \mu \mathrm{g} / \mathrm{ml}$ to remove potential RNA-bridged indirect protein-protein interactions. Subsequently, the beads were precipitated by a centrifugation at $500 \times \mathrm{g}$ and $4^{\circ} \mathrm{C}$ for 5 minutes. The beads were washed 5 times with $1 \mathrm{ml}$ cold PBSKMT and between washes a centrifugation at $500 \times \mathrm{g}$ and $4^{\circ} \mathrm{C}$ for 5 minutes was used to precipitate beads and to remove the buffer. After the last wash, the supernatants were carefully removed and the beads (samples) were resuspended in $20-50 \mu \mathrm{l} 2 \times$ SDS sample buffer.

The input controls and samples were either stored at $-20^{\circ} \mathrm{C}$ or used in western blot experiments.

RNaseA:

$10 \mathrm{mg} / \mathrm{ml}$ RNaseA

10mM Tris pH7.5

$15 \mathrm{mM} \mathrm{NaCl}$

Incubate at $100^{\circ} \mathrm{C}$ for 15 minutes

PBSKMT: see section 3.2.1.5

SDS Sample buffer: see section 3.2.1.5 


\subsubsection{Nucleo-cytoplasmic fractionation}

Cells from 200-500ml cell cultures (middle-logarithmic phase) were harvested through centrifugation at $4000 \times \mathrm{g}$ and $4^{\circ} \mathrm{C}$ for 10 minutes. The cell pellets were washed once with YPD/1M Sorbitol/2mM DTT. To digest cell walls, the cells were spheroplasted in $1-2 \mathrm{ml}$ YPD/1M Sorbitol with $0.1 \mathrm{mg}$ zymolyase per gram of cells at room temperature. The cell wall digestion was monitored under the phase contrast light microscope (Leitz Biomed Typ 020-507-010). When $50 \%$ of the cells became dark, the cells were harvested with centrifugation at $1000 \times \mathrm{g}$ and $4^{\circ} \mathrm{C}$ for 5 minutes. After washing once with $1-2 \mathrm{ml} \mathrm{YPD} / 1 \mathrm{M}$ Sorbitol, the cells were recovered in $50-100 \mathrm{ml} Y P D / 1 \mathrm{M}$ Sorbitol for 30 minutes at $25^{\circ} \mathrm{C}$. After recovery, the cells were shifted to the desired temperature for the indicated time if necessary. The cells were harvested again with centrifugation at $1000 \times \mathrm{g}$ and $4^{\circ} \mathrm{C}$ for 10 minutes. $1 / 10$ volume of the cell pellets were lysed (Yeast lysis, section 3.2.1.5) and the lysates were used as input controls. The rest of the cells were resuspended in cell lysis buffer with a volume of $0.5 \mathrm{ml}$ buffer/g cell pellets. To release the cell contents, cell buffer A was added with a volume of $1 \mathrm{ml}$ buffer/g cell pellets. The mixture was incubated for 5-10 minutes at $4^{\circ} \mathrm{C}$. Afterwards, the mixture was centrifuged at $1500 \times \mathrm{g}$ and $4^{\circ} \mathrm{C}$ for 15 minutes. The supernatant was carefully taken as the cytosolic extract. RNAs were obtained from the cytosolic extracts (Acidic phenol extraction, section 3.2.3.4) and used for qRT-PCR. Proteins were obtained from the extracts (Protein extraction and precipitation, section 3.2.2.1) and used for western blot analyses.

YPD medium: see section 3.2.1.3

Cell lysis buffer:

$18 \%(w / v)$ Ficoll 400

10mM HEPES pH6.0

Cell buffer A: 
$50 \mathrm{mM} \mathrm{NaCl}$

$1 \mathrm{mM} \mathrm{MgCl} 2$

10mM HEPES pH6.0

\subsubsection{SDS-polyacrylamide gel electrophoresis (SDS-PAGE) and western blot}

Protein samples from protein precipitation, western blot, co-immunoprecipitation or RNA co-immunoprecipitation experiments were dissolved in $1 \times$ SDS sample buffer. The samples were incubated at $95^{\circ} \mathrm{C}$ for 5 minutes followed by centrifugation at $20000 \times \mathrm{g}$ and room temperature for 1 minute. The clear supernatants were loaded onto the stacking gel. Protein ladders $(5 \mu$ l pre-stained or $10 \mu$ l unstained PageRuler Protein ladder, Thermo Fisher Scientific) were loaded to indicate the protein size. SDS-PAGE was performed in $1 \times$ SDS running buffer at $25 \mathrm{~mA}$ for the stacking gel and $35 \mathrm{~mA}$ for the resolving gel. Alternatively, a constant $6-8 \mathrm{~mA}$ was used overnight.

After SDS-PAGE, proteins were electrically transferred from the gel onto a nitrocellulose membrane. Western blot was performed by using a semi-dry electroblotter (Perfect Blue Semi dry Electroblotter, Peqlab). The SDS-polyacrylamide gel, a nitrocellulose membrane and four pieces of whatmann paper were presoaked with the transfer buffer. The transfer sandwich was assembled as follows (from the negative pole to the positive pole): two pieces of whatmann paper, the SDS-polyacrylamide gel, the nitrocellulose membrane, two pieces of whatmann paper. After removing the bubbles in the transfer sandwich, the western blot was performed at $0.8-1.2 \mathrm{~mA} / \mathrm{cm}^{2}$ for 90 to 105 minutes. After blotting, the transferred membrane was stained in the Ponceau $\mathrm{S}$ for 3 minutes followed by a washing step in $\mathrm{H}_{2} \mathrm{O}$. The Ponceau S staining stained the blotted proteins and the unstained protein ladder, which was marked. After removing residual Ponceau $S$ staining with $1 \times T$ TBST, the membrane was blocked in $5 \%(\mathrm{w} / \mathrm{v})$ milk powder/1×TBST at room temperature for 1 hour. Subsequently, the membrane was incubated with the primary antibodies (e.g. mouse anti-myc antibody, rabbit 
anti-GFP antibody, rabbit anti-Mtr4 antibody, etc.) diluted in $2 \%(\mathrm{w} / \mathrm{v})$ milk powder/1 $\times$ TBST for 2 hours at room temperature or overnight at $4^{\circ} \mathrm{C}$. After four times over 15 minutes washing with $1 \times$ TBST, the membrane was incubated with the secondary antibodies (e.g. goat anti-mouse-HRP, goat anti-rabbit-HRP, etc.) diluted in $2 \%(\mathrm{w} / \mathrm{v})$ milk powder/1×TBST for 1.5 to 2 hours at room temperature. Chemiluminescent signals were generated by using the Amersham ECL prime Western Blotting Detection reagents (GE Healthcare) according to the manufacture's instruction. The signals were detected by using the Fusion FX7 detection system (Peqlab).

$\underline{1 \times \text { SDS Sample buffer: }}$ see section 3.2.1.5

\section{5\% Stacking gel:}

$16.7 \%(v / v)$ Rotiphorese Gel 30

(aqueous $30 \%$ acrylamide and bisacrylamide stock solution at a ratio of $37.5: 1$ )

$0.125 \mathrm{M}$ Tris $\mathrm{pH} 6.8$ (recipe in section 3.1.5)

$0.1 \%(w / v)$ SDS (sodium dodecyl sulfate)

$0.1 \%(w / v)$ APS (ammonium persulfate)

$0.01 \%(v / v)$ TEMED (N,N,N,N'-Tetramethylenediamine)

8-12\% Resolving gel:

26.4\%-39.6\% (v/v) Rotiphorese Gel 30

$0.375 \mathrm{M}$ Tris $\mathrm{pH} 8.8$ (recipe in section 3.1.5)

$0.1 \%(w / v)$ SDS

$0.1 \%(w / v)$ APS

$0.04-0.08 \%(v / v)$ TEMED

$\underline{1 \times \text { SDS running buffer: }}$

$25 \mathrm{mM}$ Tris Base

$0.1 \%(w / v)$ SDS 
$192 m M$ Glycin

Transfer buffer:

192mMM glycine

$25 \mathrm{mM}$ Tris Base

$10 \%-20 \%$ methanol

$0.03 \%-0.05 \%$ SDS added if $10 \%$ methanol was used

Ponceau S solution:

$0.2 \%(w / v)$ Ponceau S

$5 \%(v / v)$ acetic acid

20×TBS:

$1 \mathrm{M}$ Tris pH7.4

$3 \mathrm{M} \mathrm{NaCl}$

$\underline{1 \times T B S T:}$

$1 \times$ TBS

$0.1 \%(v / v)$ Tween-20 


\subsubsection{Molecular biological methods}

\subsubsection{Polymerase chain reaction (PCR)}

PCR reaction parameters vary according to templates, products, and polymerases. The PCR reactions were usually set up as below:

\begin{tabular}{|l|l|l|}
\hline \multicolumn{2}{|l|}{ Component } & Final concentration \\
\hline \multirow{2}{*}{ Template } & Plasmid & $100 \mathrm{pg}-10 \mathrm{ng}$ for $50-100 \mu \mathrm{l}$ reaction \\
\cline { 2 - 3 } & or Genomic DNA & $1 \mu \mathrm{g}$ for $50-100 \mu$ l reaction \\
\hline $10 \times$ reaction buffer (polymerase specific) & $1 \times$ \\
\hline dNTP & $0.2 \mathrm{mM}$ \\
\hline DNA polymerase & $1 \mathrm{unit}$ for $50 \mu \mathrm{l}$ reaction \\
\hline Forward primer & $1 \mu \mathrm{M}$ \\
\hline Reverse primer & $1 \mu \mathrm{M}$ \\
\hline
\end{tabular}

To generate desired products, different DNA polymerases were used. For routine PCR reactions, DreamTaq DNA Polymerase (without proof-reading ability, Thermo Fisher Scientific) was used. For proof-reading PCRs (products used for e.g. sequencing, molecular cloning, etc.) either the Phusion High-Fidelity DNA Polymerase (New England Biolabs) or KAPAHiFi Polymerase (Peqlab) was used.

The PCR procedures were set as below:

\begin{tabular}{|l|l|l|l|}
\hline \multicolumn{2}{|l|}{ Step } & Temperature & Time \\
\hline \multicolumn{2}{|l|}{ Initial denaturation } & $95-98^{\circ} \mathrm{C}$ & 30 seconds to 3 minutes \\
\hline $20-40$ cycles & Denaturation & $95-98^{\circ} \mathrm{C}$ & $10-30$ seconds \\
\cline { 2 - 4 } & Annealing & $\begin{array}{l}0-5^{\circ} \mathrm{C} \text { below the Tm (DNA melting } \\
\text { temperature) of the primers }\end{array}$ & $15-60$ seconds \\
\cline { 2 - 4 } & Extension & $68^{\circ} \mathrm{C}$ or $72^{\circ} \mathrm{C}$ & $30-120$ seconds $/ \mathrm{kb}$ \\
\hline Final extension & $68^{\circ} \mathrm{C}$ or $72^{\circ} \mathrm{C}$ & $5-10$ minutes \\
\hline Hold & $4^{\circ} \mathrm{C}$ & \\
\hline
\end{tabular}




\subsubsection{DNA gel electrophoresis and gel extraction}

To prepare agarose gel $0.8 \%-1.2 \%$ agarose $(\mathrm{w} / \mathrm{v})$ was dissolved in $1 \times$ TAE buffer by cooking and mixing. Subsequently, once the agarose gels were cooled down to around $60^{\circ} \mathrm{C}$, ethidium bromide was added to a final concentration of $0.5 \mu \mathrm{g} / \mathrm{ml}$.

6×DNA loading dye was added to DNA samples to a final concentration of $1 \times$. To indicate the DNA size, 5-7 $\mu \mathrm{l}$ DNA marker $(0.5 \mu \mathrm{g} / \mu \mathrm{l}$, Lambda DNA/EcoRI+HindIII DNA Ladder or GeneRuler 1 kb DNA Ladder, Thermo Fisher Scientific) was also loaded. The DNAs were separated by agarose gelelectrophoresis at 100-120 Volts and room temperature for 20-30 minutes. DNAs were detected by the 312nm UV-light with INTAS UV-system (INTAS).

To obtain specific DNA samples, the DNA bands were cut from the gels. Desired DNAs were extracted from the gel by using the peqGOLD Gel Extraction Kit (Peqlab) according to the manufacture's instruction.

1×TAE buffer (pH8.5):

40mM Tris Base

1mM EDTA

0.114\% (v/v) Acidic acid

Adjust $\mathrm{pH}$ to 8.5

\section{6xDNA loading dye:}

10mM Tris $\mathrm{pH} 7.6$

$0.03 \%(w / v)$ Brome phenol blue

$0.03 \%(w / v)$ Xylene carbol blue

$60 \%(v / v)$ Glycerol

60mM EDTA 


\subsubsection{Genomic DNA extraction}

$10 \mathrm{ml}$ yeast culture was grown to a concentration of $>1 \times 10^{8}$ cell $/ \mathrm{ml}$. The cells were harvested through centrifugation at $4000 \times \mathrm{g}$ and room temperature for 10 minutes. The cells were mixed with $500 \mu$ l detergent lysis buffer, $500 \mu$ l phenol and $300 \mu$ l glass beads and lysed by two vigorous shakes on the Fastprep machine at $6.0 \mathrm{~m} / \mathrm{s}$ for 20 seconds.

The mixture was centrifuged at $20000 \times \mathrm{g}$ and room temperature for 5 minutes. The upper layer was carefully transferred to a fresh tube and the same volume of phenol was added. The mixture was shaken vigorously by vortexing and centrifuged at $20000 \times \mathrm{g}$ and room temperature for 5 minutes. This step was repeated twice, once replacing phenol with phenol/chloroform/isoamyl alcohol (25:24:1, Carl Roth) and once with chloroform/isoamyl alcohol (24:1, Carl Roth). Afterwards, 1/10 volume 3M sodium acetate pH5.3 and 3 times the volume of $100 \%$ ethanol were added to the extract and the mixture was incubated at $-20^{\circ} \mathrm{C}$ for 15 minutes for precipitation. Genomic DNA (gDNA) pellet was obtained by a centrifugation at $20000 \times \mathrm{g}$ and $4^{\circ} \mathrm{C}$ for 20 minutes. The pellet was washed with $75 \%$ ethanol and centrifuged at $20000 \times \mathrm{g}$ and $4^{\circ} \mathrm{C}$ for 5 minutes. The gDNA pellet was air-dried at room temperature or $37^{\circ} \mathrm{C}$ and finally dissolved in nuclease-free $\mathrm{H}_{2} \mathrm{O}$ or TE buffer pH8.0. The gDNAs were either stored at $-20^{\circ} \mathrm{C}$ or used for PCR or southern blot experiments.

\section{Detergent lysis buffer:}

$2 \%(v / v)$ Triton X-100

$1 \%(w / v)$ SDS

$100 \mathrm{mM} \mathrm{NaCl}$

$10 \mathrm{mM}$ Tris $\mathrm{Cl}$

$1 \mathrm{mM}$ EDTA

TE buffer (pH7.5 or 8.0):

$10 \mathrm{mM}$ Tris $\mathrm{pH} 7.5$ or 8.0

$1 \mathrm{mM}$ EDTA 


\subsubsection{Acidic phenol RNA extraction}

Cells from $20-50 \mathrm{ml}$ cell cultures (logarithmic phase) were harvested through centrifugation at $4000 \times \mathrm{g}$ and $4^{\circ} \mathrm{C}$ for 10 minutes. The cells were mixed with $400 \mu \mathrm{l}$ AE buffer, $40 \mu \mathrm{l} 10 \% \mathrm{SDS}$, $440 \mu \mathrm{l}$ aqua-phenol and incubated at $65^{\circ} \mathrm{C}$ for 5 minutes with occasional inversions. The mixture was frozen at $-80^{\circ} \mathrm{C}$ for more than 30 minutes and subsequently centrifuged at $20000 \times g$ at room temperature for 5 minutes. The upper phase was carefully transferred to a fresh tube.

The samples for RNA extraction were the extracts obtained as mentioned above or from the nucleo-cytoplasmic fractionation or RNA co-immunoprecipitation experiments. The same volume of aqua-phenol was added to the extract and the mixture was shaken vigorously by hand and centrifuged at $20000 \times \mathrm{g}$ and room temperature for 5 minutes. This step was repeated twice, once replacing aqua-phenol with aqua-phenol/chloroform /isoamyl alcohol (25:24:1, Carl Roth) and once with chloroform/isoamyl alcohol (24:1, Carl Roth). 1/10 the volume of $3 \mathrm{M}$ sodium acetate $\mathrm{pH} 5.3$ or $1 / 4$ the volume of $4 \mathrm{M}$ DEPC treated lithium chloride and 3 -time the volume of $100 \%$ ethanol were added to the extract and the mixture was incubated at $-80^{\circ} \mathrm{C}$ for 30 minutes or at $-20^{\circ} \mathrm{C}$ overnight for precipitation. RNA pellet was obtained by centrifugation at $20000 \times \mathrm{g}$ and $4^{\circ} \mathrm{C}$ for 30 minutes. The pellet was washed with $75 \%-20^{\circ} \mathrm{C}$ ethanol made with DEPC $-\mathrm{H}_{2} \mathrm{O}$ and centrifuged at $20000 \times \mathrm{g}$ and $4^{\circ} \mathrm{C}$ for 15 minutes. The RNA pellet was air-dried on ice and finally dissolved in DEPC- $\mathrm{H}_{2} \mathrm{O}$ or TE buffer $\mathrm{pH} 7.5$ made with DEPC- $\mathrm{H}_{2} \mathrm{O}$. The RNAs were either stored at $-20^{\circ} \mathrm{C}$ for weeks and at $-80^{\circ} \mathrm{C}$ for months or used in qRT-PCR experiments.

\section{Diethypyrocarbonate (DEPC) treated solution:}

Add DEPC in solution with final concentration of $0.1 \%$. Mix overnight and autoclave.

Alternatively, prepare solution with DEPC-treated $\mathrm{H}_{2} \mathrm{O}$

\section{AE buffer (pH 5.2):}

$50 \mathrm{mM}$ sodium acetate 
10 mM EDTA

Adjust $\mathrm{pH}$ to 5.2

TE buffer (pH7.5): see section 3.2.3.3

\subsubsection{Probe synthesis}

A nucleic acid probe can be a DNA or an RNA molecule that is labeled with e.g. the fluorescent tag Cy3 or special chemicals like digoxigenin (DIG), etc.

3'-end DIG-labelled single-strand DNA oligonucleotide probes (e.g. HK1539) for southern blot experiments were ordered from Sigma. The oligo probes were diluted in sterile $\mathrm{H}_{2} \mathrm{O}$ or TE buffer pH7.5 with a concentration of $100 \mathrm{mM}$ as stock solutions and stored at $-20^{\circ} \mathrm{C}$.

The Cy3-labelled single-strand DNA oligonucleotide probes that were labelled at both the $3^{\prime}$ and the $5^{\prime}$ ends (e.g. HK 1761, HK1789, HK1790) for fluorescent in situ hybridisation were purchased from Sigma. The oligonucleotide probes were diluted in sterile $\mathrm{H}_{2} \mathrm{O}$ or TE buffer $\mathrm{pH} 7.5$ with a concentration of $10 \mathrm{mM}$ as stock solutions and stored at $-20^{\circ} \mathrm{C}$ under light protection.

DIG labeled RNA probes for fluorescent in situ hybridisation were produced through in vitro transcription. The templates were PCR products that contained T7 transcription sites upstream of the anti-sense strands of target genes and were generated by PCR (section 3.2.3.1) and purified via DNA gel electrophoresis and gel extraction (section 3.2.3.2). The TLC1 probes were generated from the PCR products of primer pairs HK936+HK937, HK938+HK939, HK940+HK941, HK942+HK943 as templates. The 25S rRNA probes were generated from the PCR products of HK1138+HK1139. The 18S rRNA probes were generated from the PCR products of HK1140+HK1141. The U6 RNA probes were generated from the PCR products of HK1404+HK1492. To synthesize probes, 200-250ng of the PCR products were mixed with $1 \times \mathrm{T} 7$ transcription buffer (Thermo Fisher Scentific), 1u RNase inhibitor (40u/ $\mu$ l, Thermo Fisher Scentific), 5u T7 RNA polymerase (20u/ $\mu$ l, Thermo Fisher Scentific) and $1 \times$ DIG RNA labeling mix (Roche) in a total volume of $20 \mu$ l. The $20 \mu$ l-mixture was 
incubated at $37^{\circ} \mathrm{C}$ for $1.5-2$ hours. Subsequently, the reaction was stopped by adding $1 \mu \mathrm{l}$ 0.5M EDTA pH8.0. The transcripts were precipitated by adding $10 \mu \mathrm{l}$ tRNA $(10 \mathrm{mg} / \mathrm{ml}), 1 / 4$ volume 4M DEPC treated lithium chloride and $3 \times$ volume $100 \%$ ethanol. The mixture was incubated at $-20^{\circ} \mathrm{C}$ overnight or at $-80^{\circ} \mathrm{C}$ for at least 30 minutes. The RNAs were pelleted by a centrifugation at $20000 \times \mathrm{g}$ and $4^{\circ} \mathrm{C}$ for $30-60$ minutes. The pellet was washed for 10 second by using $75 \%-20^{\circ} \mathrm{C}$ ethanol (made by DEPC- $\mathrm{H}_{2} \mathrm{O}$ ) and centrifuged again at $20000 \times \mathrm{g}$ and $4^{\circ} \mathrm{C}$ for 15 minutes. The pellet was air-dried on ice. The RNAs were dissolved in $100 \mu$ DEPC- $\mathrm{H}_{2} \mathrm{O}$ and stored at $-20^{\circ} \mathrm{C}$ for weeks and at $-80^{\circ} \mathrm{C}$ for months.

TE buffer (pH7.5): see section 3.2.3.3

\subsubsection{Southern blot}

Genomic DNAs were extracted (genomic DNA extraction, section 3.2.3.3). To perform the southern blot experiments that detected telomeres, gDNAs were mixed with $1 \times$ buffer $\mathrm{R}$ (Thermo Fisher Scientific) and 10u Xhol (10u/ $\mu$, Thermo Fisher Scientific) and incubated at $37^{\circ} \mathrm{C}$ overnight. Digested gDNAs were separated overnight on $1.0-1.2 \%$ agarose gels (gel electrophoresis, section 3.2.3.2) at 2 volts/cm. Subsequently the DNA gels were depurinated in $0.25 \mathrm{~N} \mathrm{HCl}$ for 15 minutes, denatured in $0.5 \mathrm{M} \mathrm{NaOH} / 1.5 \mathrm{M} \mathrm{NaCl}$ for 30 minutes, neutralized in $0.5 \mathrm{M}$ Tris $\mathrm{pH} 7.5 / 1,5 \mathrm{M} \mathrm{NaCl}$ for 30 minutes and equilibrated in $20 \times \mathrm{SSC}$ for 15 minutes. $\mathrm{A}$ capillary transfer (dry blot) was performed as follows (from bottom to top): a glass plate (supporter), the agarose gel facing down, a dry Hybond-N+ membran (GE Healthcare), three dry whatmann papers, 5 -cm-thick paper-tower and $1 \mathrm{~kg}$ weight on the top. Alternatively, a vacuum transfer was set from bottom to top: supporter, a 20×SSC pre-soaked whatmann paper, a $20 \times$ SSC pre-soaked nylon membrane, the gel facing up and the sealing frame. The capillary transfer usually took 4 hours to overnight and the vacuum transfer took 1-2 hours. After blotting, the membranes were cross-linked under UV light $(312 \mathrm{~nm}$, Cross Linker Bio Link BLX 365) for 5 minutes and further baked at $80^{\circ} \mathrm{C}$ for 2 hours. The membranes were 
pre-hybridised at $37^{\circ} \mathrm{C}$ for 1 hour in $10 \mathrm{ml}$ hybridization buffer. Subsequently, $0.1-1.0 \mathrm{nmol}$ probes (HK1539, 100mM) were added and the membranes were hybridised at $37^{\circ} \mathrm{C}$ for 4 hours to overnight. After hybridization, the membranes were washed once with $2 \times \mathrm{SSC} / 0.1 \%$ $(\mathrm{w} / \mathrm{v}) \mathrm{SDS}, 1 \times \mathrm{SSC} / 0.1 \%(\mathrm{w} / \mathrm{v}) \mathrm{SDS}$ at room temperature, twice with $0.5 \times \mathrm{SSC} / 0.1 \%(\mathrm{w} / \mathrm{v}) \mathrm{SDS}$ at $37^{\circ} \mathrm{C}$, each for 15 minutes, respectively. The membranes were further washed with DIG washing buffer for 5 minutes and blocked in DIG blocking solution for 30 minutes at room temperature. The membranes were incubated with anti-DIG-alkaline phosphatase (Roche) diluted 1:10000 in DIG blocking solution for 30 minutes at room temperature. The membranes were washed twice with DIG washing buffer, each for 15 minutes at room temperature. The membranes were equilibrated in DIG detection buffer for 5 minutes at room temperature and incubated with the chemiluminescent substrate CSPD (Roche, 1:100 diluted in DIG detection buffer) for 5 minute at room temperature. The CSPD was removed from the membranes and the membranes were sealed in autoclave bags and incubated at $37^{\circ} \mathrm{C}$ for 15 minutes. The chemiluminescent signals were detected by using the Fusion FX7 detection system (Peqlab) (if detection time $\leq 30$ minutes) or Super RX films (Fujifilm) followed by being developed with Optimax X-Ray Film Processor (Protec) (if detection time $>30$ minutes).

20×SSC:

$3 \mathrm{M} \mathrm{NaCl}$

$300 \mathrm{mM}$ Sodium citrate

Adjust $\mathrm{pH}$ to 7.0

1M Sodium phosphate buffer ( $\mathrm{pH} 7.2)$ :

$68.4 \%(v / v) 1 \mathrm{M} \mathrm{Na}_{2} \mathrm{HPO}_{4}$

$31.6 \%(\mathrm{v} / \mathrm{v}) 1 \mathrm{M} \mathrm{NaH}{ }_{2} \mathrm{PO}_{4}$

The hybridization buffer (pH7.2) for southern blot:

0.5M sodium phosphate buffer pH7.2 (recipe in section 3.1.5) 
$7 \%(w / v)$ SDS

$1 \mathrm{mM}$ EDTA

5xMaleic acid buffer (pH7.5):

$0.5 \mathrm{M}$ Maleic acid

$0.75 \mathrm{M} \mathrm{NaCl}$

Adjust $\mathrm{pH}$ to 7.5

DIG washing buffer:

$1 \times$ Maleic acid buffer

$0.3 \%(v / v)$ tween-20

$\underline{10 \times \text { DIG blocking buffer: }}$

$1 \times$ Maleic acid buffer

$10 \%(w / v)$ blocking reagent (Roche)

DIG blocking buffer:

1:10 dilute $10 \times$ DIG blocking buffer in $1 \times$ Maleic acid buffer

DIG Detection buffer:

$0.1 \mathrm{M}$ Tris $\mathrm{pH} 9.5$

$0.1 \mathrm{M} \mathrm{NaCl}$

\subsubsection{RNA-co-immunoprecipitation (RNA-co-IP)}

The procedures of harvesting cells, lysing cells, precipitating desired proteins were similar as described in co-immunoprecipitation (section 3.2.2.2) but the PBSKMT buffer was replaced with the RNA co-IP buffer. 
After the last washing step, the beads were divided into two portions. 1/10 volume of the beads were mixed with $10 \mu \mathrm{l} 2 \times$ SDS sample buffer and used for SDS-polyacrylamide gel electrophoresis (SDS-PAGE) and western blot experiments as protein controls. The RNA was extracted from the rest $9 / 10$ volume of the beads by using acidic phenol-chloroform extraction protocol (section 3.2.3.4). The extracted RNA was stored at $-20^{\circ} \mathrm{C}$ for weeks or $-80^{\circ} \mathrm{C}$ for months or was directly used for qRT-PCR experiments.

RNA co-IP buffer: see section 3.2.1.5

SDS sample buffer: see section 3.2.1.5

\subsubsection{Quantitative reverse transcriptase PCR (qRT-PCR)}

To synthesize cDNA, $1 \mu \mathrm{g}$ RNA, $1 \times$ random hexamer primer (from 20xrandom hexamer primer, Thermo Fisher Scientifc), and nuclease-free $\mathrm{H}_{2} \mathrm{O}$ were mixed in a total volume of $11.5 \mu \mathrm{l}$. The mixtures were incubated at $65^{\circ} \mathrm{C}$ for 5 minutes and then chilled on ice for 5 minutes. $200 \mathrm{u}$ Maxima reverse transcriptase (Thermo Fisher Scientifc), 10u RNase inhibitor (Thermo Fisher Scientifc), $1 \times$ reverse transcription buffer (Thermo Fisher Scientifc) and $1 \mathrm{mM}$ dNTP (Thermo Fisher Scientifc) were added to the mixture to a final volume of $20 \mu \mathrm{l}$ and the mixture was incubated at $50-60^{\circ} \mathrm{C}$ for $30-45$ minutes. The reaction was stopped by incubation at $95^{\circ} \mathrm{C}$ for 5 minutes. The cDNA samples were either directly used in the qPCR reaction or stored at $-20^{\circ} \mathrm{C}$ for days and $-80^{\circ} \mathrm{C}$ for months.

The $25 \mu \mathrm{l}$ qPCR sample was composed of $5 \mu \mathrm{l}$ 1:50 diluted cDNA, $0.2 \mu \mathrm{l} 10 \mathrm{mM}$ reverse and forward primers, $12.5 \mu \mathrm{l} 2 \times$ GoTaq PCR master mix (Promega) and nuclease-free $\mathrm{H}_{2} \mathrm{O}$. The primers (HPLC purification level) were purchased from Sigma. The primers were diluted in nuclease-free $\mathrm{H}_{2} \mathrm{O}$ to $10 \mathrm{mM}$ and stored at $-20^{\circ} \mathrm{C}$. The primer pair HK1382+HK1384 was used to quantify the immature TLC1; the primer pair HK1385+HK1386 was used to quantify the total amount of TLC1; the primer pair HK1404+HK1405 was used to quantify the U6 snRNA; the primer pair HK1396+HK1397 was used to quantify the 18S rRNA. The PCR reactions were 
performed on the Rotor Gene Q (Qiagen). The PCR reaction was initiated with one $95^{\circ} \mathrm{C}$ 5-minute denature step. Afterwards, 40-50 cycles of two-step PCR reaction, which was composed of one $95^{\circ} \mathrm{C}$ 5-second denature step and one $60^{\circ} \mathrm{C}$ 20-second annealing and elongating step, were performed. A final melting step rising the temperature from $60^{\circ} \mathrm{C}$ to $95^{\circ} \mathrm{C}$ was used to determine the specificity of the PCR products.

Results output by Rotor Gene Q software (Qiagen) represented the Ct (cycle threshold) values, which meant the cycle number required for the fluorescent signal to cross the threshold (e.g. background level). $\mathrm{Ct}_{\text {average }}$ values were calculated from three PCR reaction replicates. $\Delta \mathrm{Ct}$ values represented the difference of transcription level between RNAs and were calculated according to " $\Delta \mathrm{Ct}=\mathrm{Ct}_{\text {averageRNA1 }}{ }^{-} \mathrm{Ct}_{\text {averageRNA2 }}$ ". Usually RNA1 indicated the transcripts of interest (e.g. total TLC1, immature $T L C 1$ ) and RNA2 indicated the reference transcripts (e.g. 18S rRNA, U6 snRNA). $\Delta \Delta \mathrm{Ct}$ values represented the different RNA transcription level between strains and were calculated according to " $\Delta \Delta \mathrm{Ct}=\Delta \mathrm{Ct}$ strain1- $-\Delta \mathrm{Ct}$ strain2". Usually strain1 indicated the strain of interest (e.g. mutants, gene-tagged strains) and strain2 indicated the reference strain (e.g. wild type, non-tagged strains). A relative fold enrichment of target RNA transcription level of target strains compared to reference strains

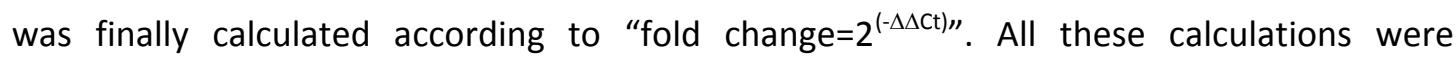
performed in Microsoft Excel (Microsoft). Calculations of standard deviations and P-values were described in Signal detection, quantification and statistical analyses (section 3.2.1.8). 


\subsubsection{Cell biological methods}

\subsubsection{Transformation}

To prepare competent E.coli cells $(\mathrm{DH} 5 \alpha)$, a single E.coli colony was grown in $10 \mathrm{ml} \mathrm{LB}$ medium at $37^{\circ} \mathrm{C}$ for overnight. Subsequently the pre-culture was further diluted in $250 \mathrm{ml}$ $\mathrm{SOB}$ medium and grown untill the $\mathrm{OD}_{600}$ reached 0.6 . The culture was aliquoted into $50 \mathrm{ml}$ falcons and the aliquots were incubated on ice for 10 minutes. The E.coli cells were harvested through centrifugation at $4000 \times \mathrm{g}$ and $4^{\circ} \mathrm{C}$ for 15 minutes. The cells were washed once in $80 \mathrm{ml}$ ice-cold transformation buffer, incubated 10 minutes on ice and harvested through centrifugation at $4000 \times \mathrm{g}$ and $4^{\circ} \mathrm{C}$ for 15 minutes. The cells were resuspended in $20 \mathrm{ml}$ ice-cold transformation buffer/7.5\% (v/v) DMSO. The mixtures were incubated on ice for 10 minutes and aliquoted to $500 \mu$ l before they were frozen in liquid nitrogen. These ultracompentent E.coli cells were stored at $-80^{\circ} \mathrm{C}$.

To transform E.coli cells, plasmids were incubated with the ultracompentent cells on ice for 30 minutes followed by a 2 -minute heat shock at $42^{\circ} \mathrm{C}$. The E.coli cells were subsequently shaken in $1 \mathrm{ml}$ LB medium for 45 minutes to 1.5 hours at $37^{\circ} \mathrm{C}$. The cells were finally harvested at $20000 \times \mathrm{g}$ and room temperature for 1 minute and were grown on specific selective plates (e.g. LB plate containing ampicillin, etc.).

LB medium: see section 3.2.1.3

\section{$\underline{\text { SOB medium: }}$}

$0.5 \%(\mathrm{w} / \mathrm{v})$ Yeast extract

2\% (w/v) Peptone

$10 \mathrm{mM} \mathrm{NaCl}$

$2.5 \mathrm{mM} \mathrm{KCl}$

$10 \mathrm{mM} \mathrm{MgCl} 2$

$10 \mathrm{mM} \mathrm{MgSO}_{4}$ 


\section{Transformation buffer:}

10mM HEPES pH 6.3

$15 \mathrm{mM} \mathrm{CaCl} 2$

$55 \mathrm{mM} \mathrm{MnCl} 2$

$250 \mathrm{mM} \mathrm{KCl}$

To transform yeast cells, the cells from $10-20 \mathrm{ml}$ cell culture (logarithmic phase) were harvested by a centrifugation at $4000 \times \mathrm{g}$ and room temperature for 10 minutes. The cells were washed once with $1 \mathrm{ml}$ sterile $\mathrm{H}_{2} \mathrm{O}$ and resuspended to $1 \times 10^{9}$ cells $/ \mathrm{ml}$ in lithium acetate

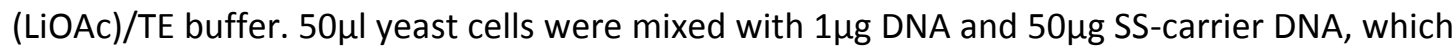
was boiled at $95^{\circ} \mathrm{C}$ for 5 minutes and chilled on ice for 2 minutes before usage. $300 \mu l$ PEG400/LiOAC/TE buffer was added to the mixtures and the mixtures were incubated at $25^{\circ} \mathrm{C}$ for 30 minutes to 2 hours. The cells were heat-shocked at $42^{\circ} \mathrm{C}$ for 15 minutes and washed in $1 \mathrm{ml}$ sterile $\mathrm{H}_{2} \mathrm{O}$. The cells were harvested with centrifugation at $10000 \times \mathrm{g}$ and room temperature for 1 minute and resuspended in $100 \mu \mathrm{l} \mathrm{H}_{2} \mathrm{O}$. The transformed cells were grown on the selective plates (e.g. URA- plates, LEU- plates according to the select markers).

\section{Lithium acetate (LiOAc)/TE buffer (pH7.5):}

100mM Lithium acetate

10mM Tris pH7.5

$1 \mathrm{mM}$ EDTA

\section{PEG/ Lithium acetate (LiOAc)/TE buffe (pH7.5)r:}

$40 \%(v / v)$ PEG4000

100mM Lithium acetate

10mM Tris pH7.5

$1 \mathrm{mM}$ EDTA 


\subsubsection{Immunofluorescence (IF)}

Cells were grown to the middle-logarithmic phase if not indicated otherwise. If necessary, the cells were shifted to the desired temperature $\left(e . g .16^{\circ} \mathrm{C}\right.$ for some cold sensitive strains, $37^{\circ} \mathrm{C}$ for some heat sensitive strains, etc.) for the indicated time (usually 30 to 60 minutes). The cells were subsequently fixed by adding formaldehyde (37\%) to a final concentration of $4 \%$ $(\mathrm{v} / \mathrm{v})$. After fixation for 30 to 60 minutes, the cells were washed once with $1 \mathrm{ml}$ potassium phosphate buffer pH6.5 and twice with $1 \mathrm{ml}$ P-solution. The cells were resuspended and incubated in $100 \mu \mathrm{l}$ P-solution/10mM DTT for 10 minutes. Furthermore, the cell walls were digested by adding $5 \mu 110 \mathrm{mg} / \mathrm{ml}$ zymolyase (Sigma). The cell wall digestion was monitored under the phase contrast microscope (Leitz Biomed Typ 020-507-010). When over 70\% cells became dark, the spheroplasts were harvested by centrifugation at $500 \times \mathrm{g}$ and $4^{\circ} \mathrm{C}$ for 5 minutes. The cells were washed twice with $500 \mu \mathrm{l}$-solution and resuspended in P-solution with a concentration of around $1 \times 10^{9} \mathrm{cell} / \mathrm{ml}$. On poly-L-lysine coated slides $30 \mu \mathrm{l}$ cells suspensions were incubated with the wells for 20 to 30 minutes at room temperature. After the cells were permeabilised in P-solution/0.5\% (v/v) triton-X100, the cells were blocked in antibody blocking buffer for 1 hour at room temperature. Subsequently, the cells were incubated with the indicated primary antibodies (e.g. mouse anti-GFP antibody, rabbit anti-myc antibody, etc.), which were diluted in an appropriate concentration in antibody blocking buffer, for 2 hours at room temperature or overnight at $4^{\circ} \mathrm{C}$. The primary antibodies were removed by washing for four times over 15 minutes with antibody blocking buffer. The cells were incubated with indicated secondary antibodies (e.g. sheep anti-mouse-AlexaFluor488 antibody, sheep anti-rabbit-AlexaFluor488 antibody, etc.), which were diluted to the appropriate concentration in antibody blocking buffer, at room temperature for 1.5-2 hours. Afterwards, the slides were washed with antibody blocking buffer for four times 15 minutes followed by washing twice for 30 minutes with $1 \times$ PBT. The cells were incubated with hoechst 33342 (1:10000 diluted in 1×PBS, Sigma) for 2-3 minutes to stain the DNA. Finally the slides were washed with $1 \times$ PBS for 3 times 5 minutes and covered with mounting solution (recipe in section 3.1.5) and coverslips. The slides were 
either immediately analysed under microscope or were stored at $-20^{\circ} \mathrm{C}$ for months.

Potassium phosphate buffer (pH6.5): see section 3.2.1.4

P-solution: see section 3.2.1.4

10×PBS: see section 3.2.1.5

$\underline{1 \times P B T:}$

$1 \times$ PBS

$0.1 \%(v / v)$ Tween-20

Antibody Blocking Buffer:

$5-10 \%(v / v)$ heat inactive fetal bovine serum

$1 \times$ PBT

Mounting solution:

$2 \%(w / v) n$-Propyl gallate

$80 \%(v / v)$ Glycerol

$1 \times$ PBS

\subsubsection{RNA fluorescent in situ hybridization (FISH)}

Cells were fixed, washed, zymolyase digested, immobilized on poly-L-lysine coated slides and permealized as described in immunofluorescence (section 3.2.4.2). The cells were pre-hybridised in hybridisation buffer at $37^{\circ} \mathrm{C}$ for 1 hour. After pre-hybridisation, the appropriate probes were diluted (1:20-1:40 for DIG labeled RNA probes, 1:400-1:4000 for Cy3 labeled oligo probes) in hybridisation buffer and were incubated on the cells at $37^{\circ} \mathrm{C}$ overnight. The cells were washed once with $2 \times \mathrm{SSC}$ for 1 hour at room temperature, once with $1 \times$ SSC for 1 hour at room temperature, once with $0.5 \times$ SSC for 30 minutes at $37^{\circ} \mathrm{C}$ and 
once with $0.5 \times \mathrm{SSC}$ for 30 minutes at room temperature.

If Cy3 labeled oligo probes were used, the cells were incubated with hoechst33342 (Sigma) diluted 1:10000 in $1 \times$ PBS for 2-3 minutes and washed 3 times with $1 \times \mathrm{PBS}$, each for 5 minutes.

Alternatively, if DIG labeled RNA probes were used, the cells were blocked in antibody blocking buffer for 1 hour at room temperature. The cells were incubated with anti-DIG-FITC antibodies (Roche), which were diluted 1:200 in antibody blocking buffer, at $4^{\circ} \mathrm{C}$ for overnight. Afterwards, the unbound antibodies were removed by washing the cells twice with antibody blocking buffer for 15 minutes, once with antibody blocking buffer for 30 minutes and once with PBT for 30 minutes. The cells were incubated with hoechst33342 (Sigma) diluted 1:10000 in 1×PBS for 2-3 minutes and were washed 3 times with $1 \times$ PBS, each for 5 minutes.

The slides were finally covered with the mounting solution and coverslips and were either stored at $-20^{\circ} \mathrm{C}$ or analysed under microscope.

\section{$\underline{50 \times \text { Denhardt's: }}$}

$1 \%(w / v)$ Ficoll

$1 \%(w / v)$ Polyvinylpyrorolidone

$1 \%(w / v) B S A$

20×SSC: see section 3.2.3.6

hybridisation buffer:

$50 \%(v / v)$ deionised formamide

$5 \times$ DEPC treated SSC

$1 \times$ Dehardt's

$0.1 \mathrm{mg} / \mathrm{ml} \mathrm{Heparin}$

Made by DEPC treated $\mathrm{H}_{2} \mathrm{O}$ 
$\underline{1 \times P B S:}$ see section 3.2.1.5

1×PBT: see section 3.2.4.2

Antibody Blocking Buffer: see section 3.2.4.2

Mounting solution: see section 3.2.4.2 


\section{Results}

\subsection{TLC1 Transport}

\subsubsection{TLC1 nuclear export}

\subsubsection{Fluorescent in situ hybridization experiments reveal that the nuclear export of TLC1 requires the mRNA export machinery}

As a non-coding RNA (ncRNA) and a product of the RNA polymerase II, TLC1 shares many features with the other ncRNAs from RNA polymerase II, e.g. snRNAs, snoRNAs etc. In mammalian cells, RNA polymerase II synthesised ncRNAs utilise CRM1 for their nuclear export (Kohler and Hurt, 2007). In Saccharomyces cerevisiae, TLC1 also utilises the Crm1/Xpo1 export receptor for its export (Gallardo et al., 2008). However, whether there are other factors involved in TLC1 nuclear export still remains unknown. Since an mRNA export factor, Mex67, is involved in rRNA export together with Crm1/Xpo1 (Faza et al., 2012; Gadal et al., 2001; Ho et al., 2000; Hurt et al., 1999; Stage-Zimmermann et al., 2000; Yao et al., 2007), it is unclear whether this is the same case for TLC1 export. To further investigate TLC1 nuclear export, fluorescent in situ hybridisation experiments were performed to identify TLC1 localisation in the mRNA export mutants mex67-5, rat7-1 and rat8-2 (figure 4.1.1).

Prior to fluorescent in situ hybridisation experiments, the cell cultures of wild type (HKY381), xpo1-1 (HKY206), mex67-5 (HKY644), rat7-1 (HKY124) and rat8-2 (HKY130) strains were grown to the middle-logarithmic phase and the cells were shifted to $37^{\circ} \mathrm{C}$ for 1 hour. After the cells were spheroplasted, permeabilized and immobilized on slides, the cells were pre-hybridised. Afterwards, DIG labelled specific TLC1 RNA probes were applied and the hybridised TLC1 RNAs were subsequently detected by FITC conjugated anti-DIG antibodies. 


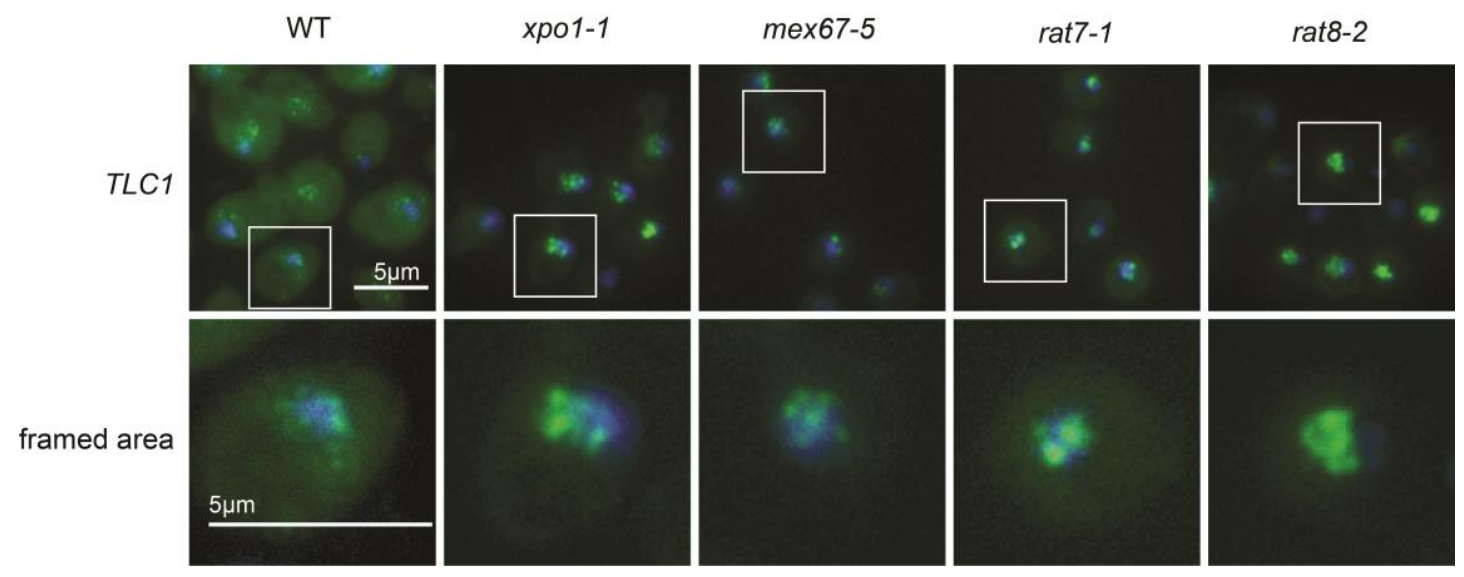

Figure 4.1.1 TLC1 localization patterns in the mRNA export defective mutants are similar to that seen in the xpo1-1 cells. To identify $T L C 1$ localisation in mRNA export mutants, fluorescent in situ hybridization (FISH) experiments were performed in the indicated strains. All cells were grown to the logarithmic phase and shifted to $37^{\circ} \mathrm{C}$ for 1 hour. Subsequently, in-situ hybridisation was performed by using specific DIG labeled anti-sense RNA probes against TLC1 and the hybridised TLC1 molecules were detected with FITC labeled anti-DIG antibodies (green). The nuclei were stained with hoechst33342 (blue). The framed areas show enlarged single cells in the figures below. At least three independent experiments were done, one of which is shown here. Scale bars: $5 \mu \mathrm{m}$.

The results (figure 4.1.1) show that in wild type cells, TLC1 signals are clustered in 8-10 foci around the nucleus as shown in previously published data (Gallardo et al., 2008) but the cytoplasmic background is relatively high, reflecting a low abundance of TLC1 in the cytoplasm due to its nucleo-cytoplasmic trafficking (Ferrezuelo et al., 2002; Gallardo et al., 2008). Differently, the xpo1-1 mutant, in which TLC1 export is inhibited (Gallardo et al., 2008), shows a more concentrated TLC1 nuclear accumulation and the cytoplasmic signals are much weaker than those in wild type cells. Strikingly, in the mRNA export mutants, mex67-5, rat7-1 and rat8-2, the phenotypes of TLC1 localisation are more similar to that in xpo1-1 than in wild type cells, suggesting a potential role of the mRNA transport pathway in TLC1 nuclear export.

Nevertheless, due to the nature of the nucleo-cytoplasmic trafficking of TLC1 (Ferrezuelo et al., 2002; Gallardo et al., 2008), the localisation patterns of TLC1 in mex67-5, rat7-1 and rat8-2 cells can also be due to enhanced TLC1 nuclear re-import. To distinguish whether the nuclear accumulation of TLC1 in these mutants was due to nuclear export block or increased nuclear re-import, these mRNA export defective mutants were crossed with the $y k u 70 \Delta$ strain. In this background, TLC1 is retained in the cytoplasm after its nuclear export (Gallardo 
et al., 2008).

Fluorescent in situ hybridisation experiments were performed as described above in wild type (HKY381), xpo1-1 (HKY206), yku704 (HKY1073), mex67-5 yku70 4 (HKY1397), rat7-1 $y k u 70 \Delta$ (HKY1398) and rat8-2 yku70 4 (HKY1396), to detect the localisation of TLC1 (figure 4.1.2). Additionally, a Cy3 labelled oligo-d(T) $)_{50}$ probe was used to identify the localisation of poly $(A)^{+}$containing RNAs in these strains. Since the probes were labelled with the fluorescent dye $\mathrm{Cy} 3$, the fluorescent in situ hybridisation experiments without the antibody detection step were performed.

Compared to the TLC1 distribution in the cytoplasm of the $y k u 70 \triangle$ strain, TLC1 accumulates in the nuclei of the mRNA export mutants (HKY1397, HKY1398, HKY1396) (TLC1 in figure 4.1.2). Besides, the localisation patterns of TLC1 in these mutants remained comparable to that seen in xpo1-1, confirming TLC1 nuclear export impairment in the mRNA export mutants. Besides, the fluoresecent in situ hybridisation experiments that detect $\operatorname{poly}(A)^{+}$
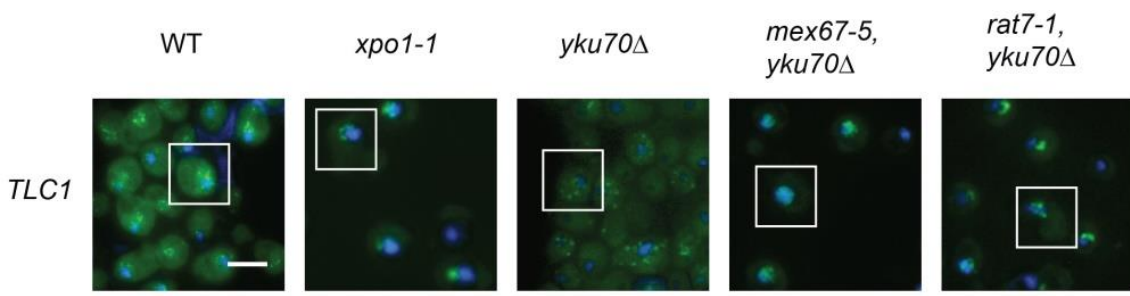

$$
\text { rat8-2, }
$$
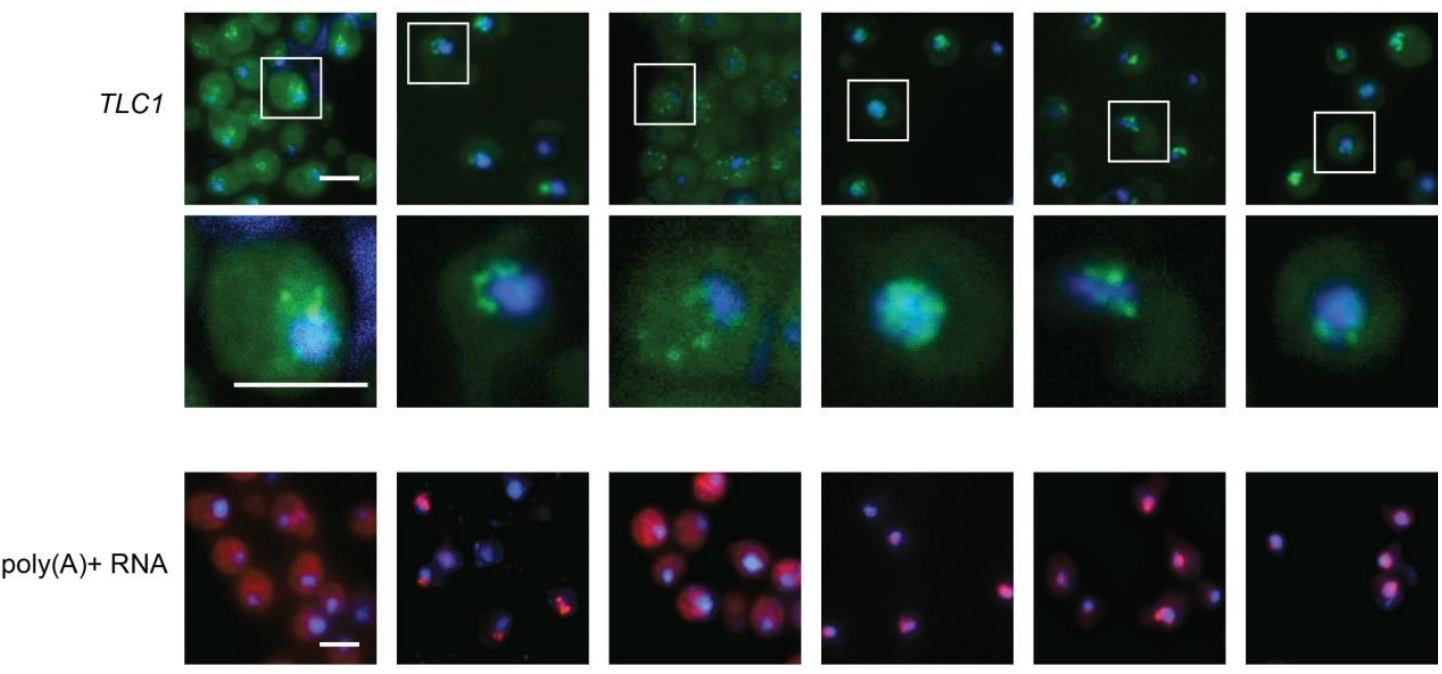

Figure 4.1.2 No TLC1 cytoplasmic retention is observed in the mRNA export defective mutants that contain the yku70 $\Delta$ background. Fluorescent in situ hybridization (FISH) experiments were performed in the indicated strains. All strains were grown to the logarithmic phase and shifted to $37^{\circ} \mathrm{C}$ for 1 hour. Subsequently, in-situ hybridisation was performed by using specific DIG labeled anti-sense RNA probes against TLC1 and the hybridised TLC1 molecules were detected with FITC labeled anti-DIG antibodies (green). Poly $(A)^{+}$RNA was detected by using a CY3 labeled oligo $d(T)_{50}$ probe (red). The nuclei were stained with hoechst33342 (blue). The framed areas show enlarged single cells in the figures below. At least three independent experiments were done, one of which is shown here. Scale bars: $5 \mu \mathrm{m}$. 
RNAs (poly $(A)^{+}$RNA in figure 4.1.2) showed that the poly $(A)^{+}$RNAs accumulate in the mRNA export mutants. Surprisingly, in the xpo1-1 mutant, poly $(A)^{+}$RNA nuclear export defect was observed. This phenotype was also mentioned in earlier published literature and was proposed as an indirect effect of the defective Xpo1 (AskjaerStade et al., 1997).

Summed up, these results extend the previous knowledge that TLC1 is exported via Crm1/Xpo1 and suggest a possibility of TLC1 nuclear export via the mRNA export machinery. 


\subsubsection{Nucleo-cytoplasmic fractionation shows decreased cytoplasmic abundance of TLC1 upon blocking the mRNA export pathway}

Due to the TLC1 nucleo-cytoplasmic movement (Ferrezuelo et al., 2002; Gallardo et al., 2008), its export block in the export mutants was proposed to reduce the amount of cytoplasmic TLC1. To examine this hypothesis, a cytoplasmic cell fractionation was performed and the amount of the cytoplasmic TLC1 was measured via qRT-PCR (figure 4.1.3).

The strains wild type (HKY381), sup45-2 (HKY446), xpo1-1 (HKY206), mex67-5 (HKY644), rat7-1 (HKY124) and rat8-2 (HKY130) were grown to the logarithmic phase. The cells were spheroplasted and further split into two equal portions. One portion was incubated at $25^{\circ} \mathrm{C}$ and the other portion was shifted to $37^{\circ} \mathrm{C}$ for 1 hour. Cells were lysed and subsequently fractionated. Zwf1 and Nop1 were used as the indicators of the cytoplasmic and nuclear fraction, respectively. Zwf1, a cytoplasmic glucose-6-phosphate dehydrogenase, and Nop1, a nucleolar histone glutamine methyltransferase, were detected through western blot analyses by using anti-Zwf1 or anti-Nop1 antibodies, respectively. The cytoplasmic RNAs were extracted from the cellular cytoplasmic factions. TLC1 was quantified using a specific TLC1 primer pair (HK1385+HK1386) through qRT-PCR. Wild type and the sup45-2 strain, a translation termination mutant that should not be involved in non-coding RNA transport, were used as the reference strains in these experiments. To evaluate the level of cytoplasmic TLC1, the amount of the cytoplasmic TLC1 was compared to that of the total $T L C 1$. These ratios were set into relation to that of wild type at $25^{\circ} \mathrm{C}$.

The western blot analyses show an exclusive detection of Zwf1 but not Nop1 in the cytoplasmic factions ( $\mathrm{C}$ in figure 4.1.3 upper panel) confirming a successful isolation of the cytoplasmic fractions. Furthermore, the qRT-PCR analyses show that in xpo1-1 and in the mRNA export defective mutants, the cytoplasmic TLC1 levels are as stable as those in 


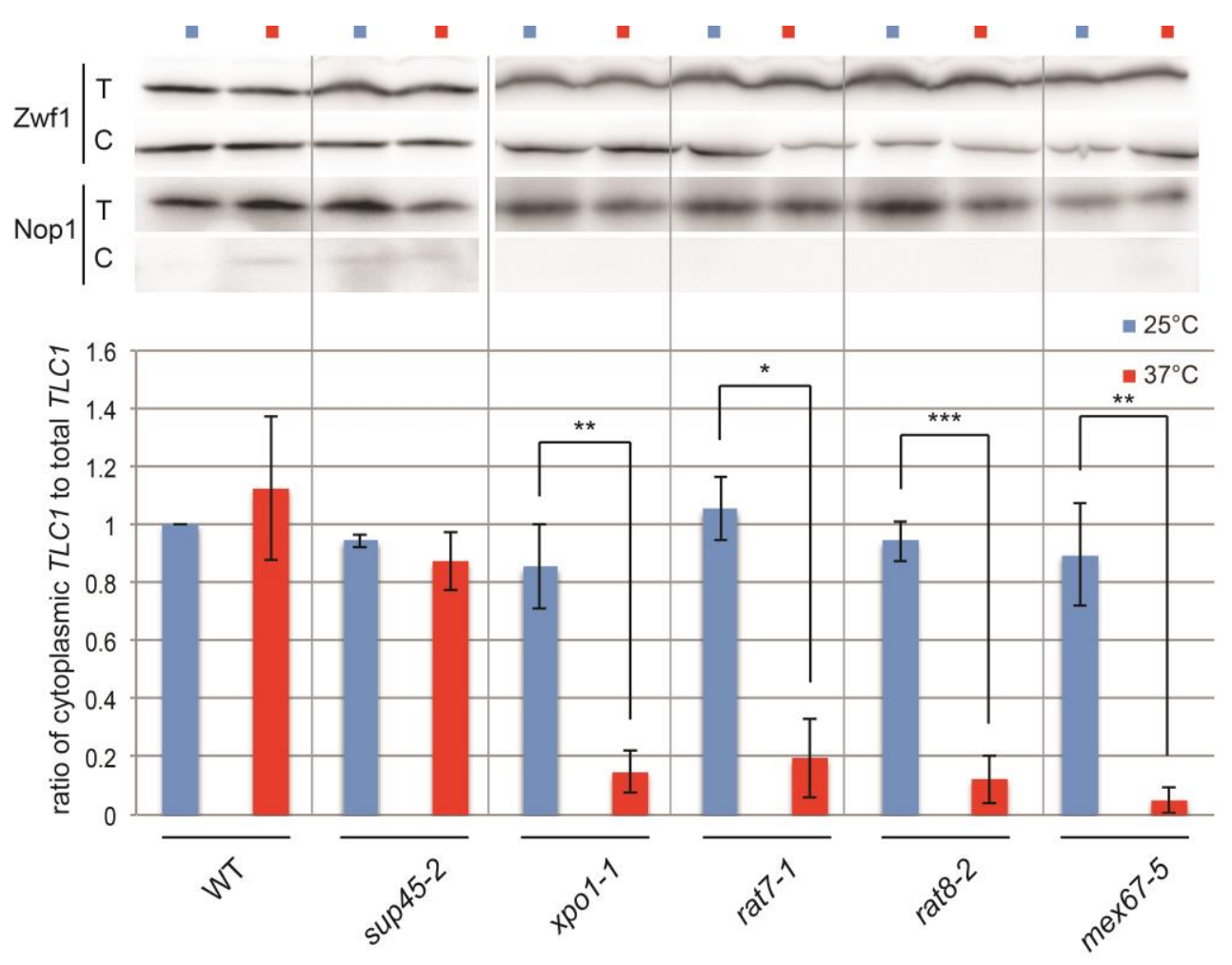

Figure 4.1.3 Analyses of the cytoplasmic fraction show a reduction of the cytoplasmic TLC1 level in the export defective mutants. The indicated strains were grown to the logarithmic phase. Half of the cells were shifted to $37^{\circ} \mathrm{C}$ for 1 hour and the other half was kept at $25^{\circ} \mathrm{C}$ for 1 hour. The cytoplasmic fractions were extracted and confirmed through western blot analyses by detecting the cytoplasmic protein Zwf1 and the nuclear protein Nop1 (C in upper panel). The total cell lysates were used as the loading controls ( $T$ in upper panel). After RNA was extracted from the cytoplasmic fractions and the total cell lysates, cytoplasmic TLC1 and total TLC1 were measured through qRT-PCR by using the primer pair HK1385+HK1386. The amount of cytoplasmic TLC1 was compared to that of the total TLC1. These ratios were set into relation with that of wild type at $25^{\circ} \mathrm{C}$ and shown in the diagram $\left(25^{\circ} \mathrm{C}\right.$ : blue column/dot; $37^{\circ} \mathrm{C}$ : red column/dot). At least three independent experiments were done. Error bars indicate the standard deviation. P-value was calculated using two-tailed, paired t-test $\left(^{*}: 0.01 \leq p \leq 0.05\right.$; **: $0.001 \leq p \leq 0.01 ; * * *: 0.0001 \leq p \leq 0.001)$

wild type and sup45-2 at $25^{\circ} \mathrm{C}$ (blue columns in figure 4.1.3). Nevertheless, compared to wild type and sup45-2, the cytoplasmic amount of TLC1 was largely reduced ( 80\%-90\%) in xpo1-1 and in the mRNA export defective mutants at $37^{\circ} \mathrm{C}$ (red columns in figure 4.1.3). These results provide a direct evidence that in the mRNA export mutants, nuclear export of TLC1 is also impaired, further suggesting the involvement of the mRNA export factors in TLC1 nuclear export. 


\subsubsection{The mRNA export factors physically interact with TLC1}

If the mRNA export factors were involved in TLC1 nuclear export, these factors should also physically interact with TLC1. To test this hypothesis, RNA co-IP experiments were performed (figure 4.1.4).

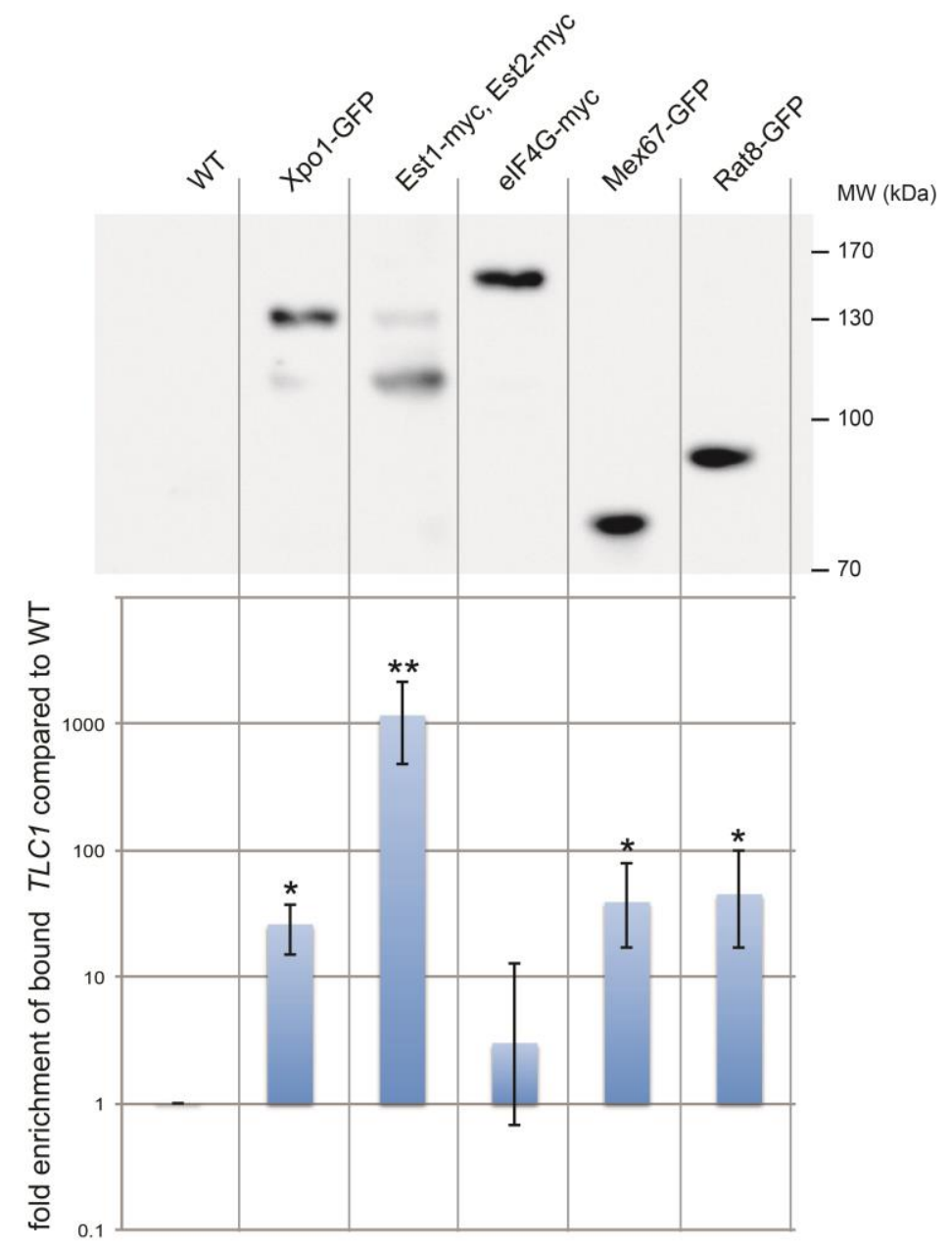

Figure 4.1.4 TLC1 physically interacts with the mRNA export factors. All strains wild type (HK381), XPO1/CRM1-GFP (HKY145), EST1-MYC EST2-MYC (HKY1292), TIF4631/EIF4G-MYC (HKY578), MEX67-GFP (HKY1266) and RAT8/DBP5-GFP (HKY1242) were grown to the logarithmic phase. Proteins of interest were immuno-precipitated by using the mixture of anti-myc and anti-GFP antibodies and confirmed through western blot analyses (top panel). RNA bound to these proteins and the total RNA were extracted. TLC1 was quantified though qRT-PCR using the specific TLC1 primer pair (HK1385+HK1386). The amount of protein bound TLC1 was compared to that of the total TLC1. These ratios were set into relation to that of wild type and shown in the diagram (bottom panel). At least three independent experiments were done. Error bars indicate the standard deviation. P-value was calculated according to two tailed, paired t-test $\left(*\right.$ : $0.01 \leq \mathrm{p} \leq 0.05$; ${ }^{* *}$; $0.001 \leq p \leq 0.01)$. 
In these experiments the strain wild type (HKY381) and the genomically tagged strains XPO1/CRM1-GFP (HKY145), EST1-MYC EST2-MYC (HKY1292), TIF4631/EIF4G-MYC (HKY578), MEX67-GFP (HKY1266) and RAT8/DBP5-GFP (HKY1242) were cultivated to the middle-logarithmic phase. The cells were harvested and lysed. The myc or GFP tagged proteins were precipitated using specific antibodies (anti-myc or anti-GFP). The RNA bound to these proteins was eluted. Subsequently, TLC1 was quantified through qRT-PCR analyses (primer pair HK1385+HK1386) to reveal the physical interaction between TLC1 and the analysed factors. To evaluate the interaction levels between the factors and TLC1, the amount of TLC1 bound to these factors was compared to that of the total TLC1 and the ratios were set into relation to that of wild type. The precipitated proteins were controlled via western blot analyses. (figure 4.1.4 upper panel).

As demonstrated in figure 4.1.4, Est1 and Est2 show a strongly enriched TLC1 binding ( 1000 fold) compared to the non-tagged background control, wild type (set as baseline 1). This suggests a specific and enduring interaction between the telomerase components and TLC1. On the other side, elF4G, a translation initiation factor that is proposed to have no connection with non-coding RNA transport, shows an expected low TLC1 binding ability that is similar to the non-tagged wild type control. Furthermore, similar to Xpo1 ( 26 fold enrichment compared to the non-tagged wild type), two mRNA export factors, Mex67 as well as Dbp5/Rat8, showed a significantly enhanced TLC1 binding ( 39 fold for Mex67 and 37 fold for Dbp5/Rat8), suggesting an interaction between TLC1 and these factors. Although there is no evidence for a direct interaction, the results suggest an involvement of the mRNA export factors in TLC1 nuclear export. Besides, together with previous results showing that TLC1 molecules accumulate in the nuclei of the mRNA export mutants, mex67-5, rat7-1 and rat8-2, it can also be concluded that $T L C 1$ nuclear export does not only depended on a single factor, e.g. Mex67, but rather require several proteins, including Mex67, Rat8/Dbp5 and Rat7. 


\subsubsection{The TLC1 nuclear export block observed in mRNA export mutants is not due to impaired TLC1 transcription or maturation}

TLC1 needs a series of processing steps to reach maturation and some of these processing failures lead to a $T L C 1$ nuclear mislocalisation, e.g. defect in hypermethylation of the $m^{7} G$ cap structure of TLC1 (Gallardo et al., 2008). Therefore, the nuclear accumulation of TLC1 might also be caused by defective TLC1 transcription or processing. To exclude this possibility, qRT-PCR experiments were performed to examine TLC1 transcription and processing in these mRNA export mutants.

The strains wild type (WT, HKY381), xpo1-1 (HKY206), rat7-1 (HKY124), rat8-2 (HKY130) and mex67-5 (HKY644) were grown to the logarithmic phase and subsequently shifted to $37^{\circ} \mathrm{C}$ for 1 hour. Total RNA was extracted from the cells. Specific primer pairs that detect unprocessed TLC1 (HK1382+HK1384) and total TLC1 (both processed and unprocessed, HK1385+HK1386) were designed as shown in figure 4.1.5A. As demonstrated in the figure, the primer pair for the total TLC1 (HK1385+HK1386) flanks the region that includes the template of reverse transcription for telomere elongation and a part of the Est2 binding domain. The products of the primer pair for unprocessed TLC1 (HK1382+HK1384) contain a part of the sequence, which will be post-transcriptionally removed during TLC1 maturation, and a part of the matured $T L C 1$, which bears the $\mathrm{Sm}_{7}$ binding site. Besides, the primer pair HK1404+HK1405 was used to quantify the U6 snRNA, an RNA Polymerase III product, as a reference for the qRT-PCR experiments. To evaluate the transcription levels of $T L C 1$, the amount of total TLC1 was compared to that of the snRNA U6 and the ratios were set into relation to that of the wild type. To evaluate TLC1 processing, the amount of unprocessed TLC1 was compared to that of the total TLC1 and the ratios were set into relation to that of the wild type. 
A

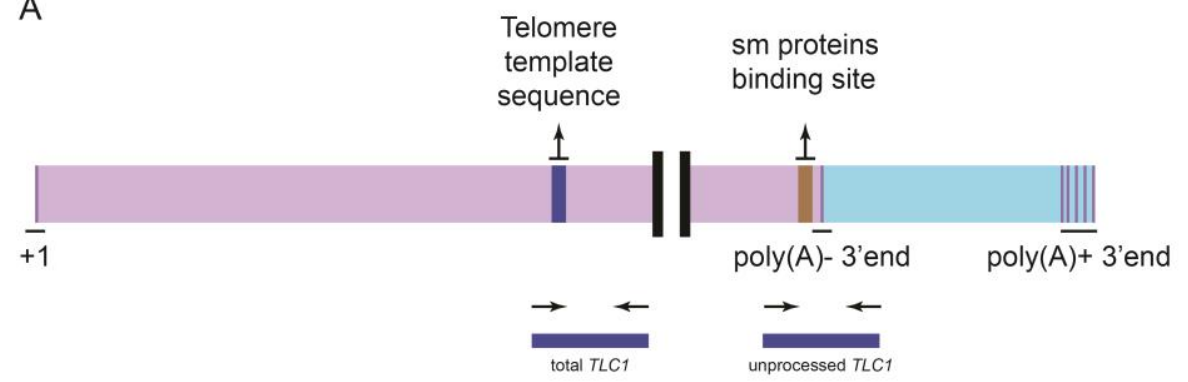

B

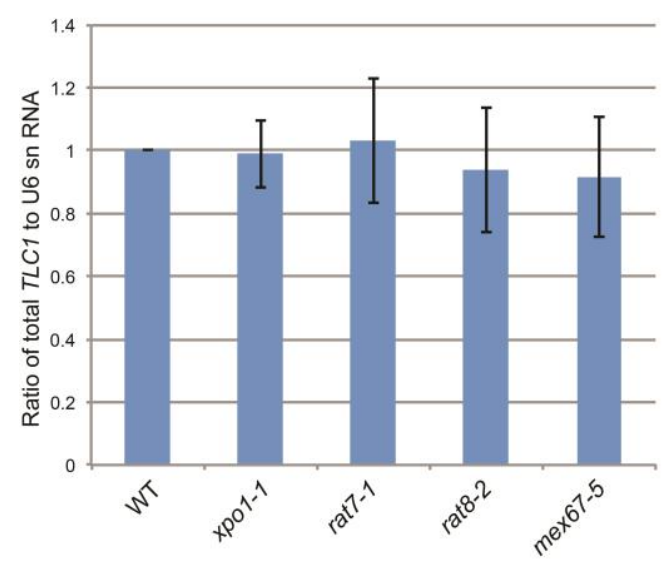

C

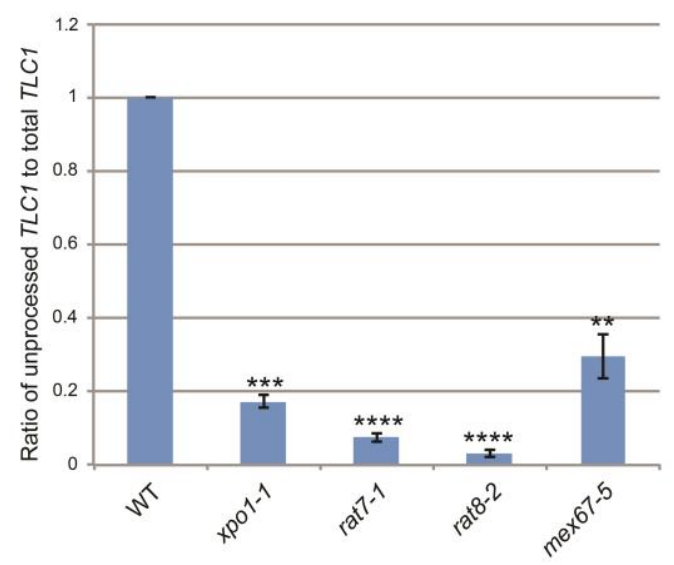

Figure 4.1.5 TLC1 nuclear export defect in mRNA export mutants is not due to impairments on its transcription and processing. (A) A brief illustration of the TLC1 structure and the schematic demonstration of the primer pairs. The primer pair (HKY1385+HK1386) for detecting total TLC1 covers the TLC1 core region including the telomere template sequence. The primer pair (HK1382+HK1384) for detecting immature TLC1 flanks the $3^{\prime}$-end of mature TLC1 and the $5^{\prime}$-part of the region that is removed from immature TLC1 during processing steps. (B,C) All indicated strains were shifted to $37^{\circ} \mathrm{C}$ for 1 hour. Subsequently, total RNA was extracted from the cells. Total TLC1 and unprocessed TLC1 were quantified through qRT-PCR using their specific primer pair (HK1385+HK1386 and HK1382+ HK1394), respectively. The U6 snRNA, as the reference RNA, was quantified by using the specific primer pair (HK1404+HK1405). The amount of the total TLC1 was compared to that of the snRNA U6 and the ratios were set into relation to that of the wild type (B). The amount of the unprocessed TLC1 was compared to that of the total TLC1 and the ratios were set into relation to that of the wild type (C). At least three independent experiments were done. Error bars indicate the standard deviation. $\mathrm{P}$ value was calculated via two-tailed, paired t-test ${ }^{* *}$ : $0.001 \leq p \leq 0.01 ; * * *: 0.0001 \leq p \leq 0.001 ; * * * *: p<0.0001)$.

The results show that the relative transcription levels of TLC1 compared to the U6 SnRNA remain unchanged in all export defective mutants compared to the wild type (figure 4.1.5B). Furthermore, although the rates of unprocessed $T L C 1$ show unexpected reductions in the mRNA export defective mutants (figure 4.1.5C), these reductions are similar within the mRNA export mutants and the known TLC1 export mutant, xpo1-1. These identical 
phenotypes in the mRNA export mutants and xpo1-1 further suggest that these factors might possess similar functions on TLC1 in mediating its nuclear export. Moreover, these results are consistent with previous findings that the maturation of $T L C 1$, including hypermethylation and 3'-trimming, occurs in the nucleus via the nuclear RNA modification machinery, e.g. Tgs1 (Franke et al., 2008) and the NRD pathway (Coy et al., 2013; Noel et al., 2012). In addition, fluorescent in situ hybridisation experiments showing that TLC1 does not co-localise with the nucleolus in the export defective mutants (Daniel Becker and Heike Krebber) suggest an exit from the processing sites. All of these results support a model in which $T L C 1$ undergoes the maturation steps prior to its nuclear export. 


\subsubsection{The TLC1 nuclear export is mediated through cooperation of Xpo1/Crm1 and the mRNA export factor}

The results above show that the TLC1 nuclear export is mediated not only by the Crm1/Xpo1 export receptor, but also by the mRNA export factors. However, the relationship between these two pathways in the role of TLC1 translocation is still unclear. To determine whether

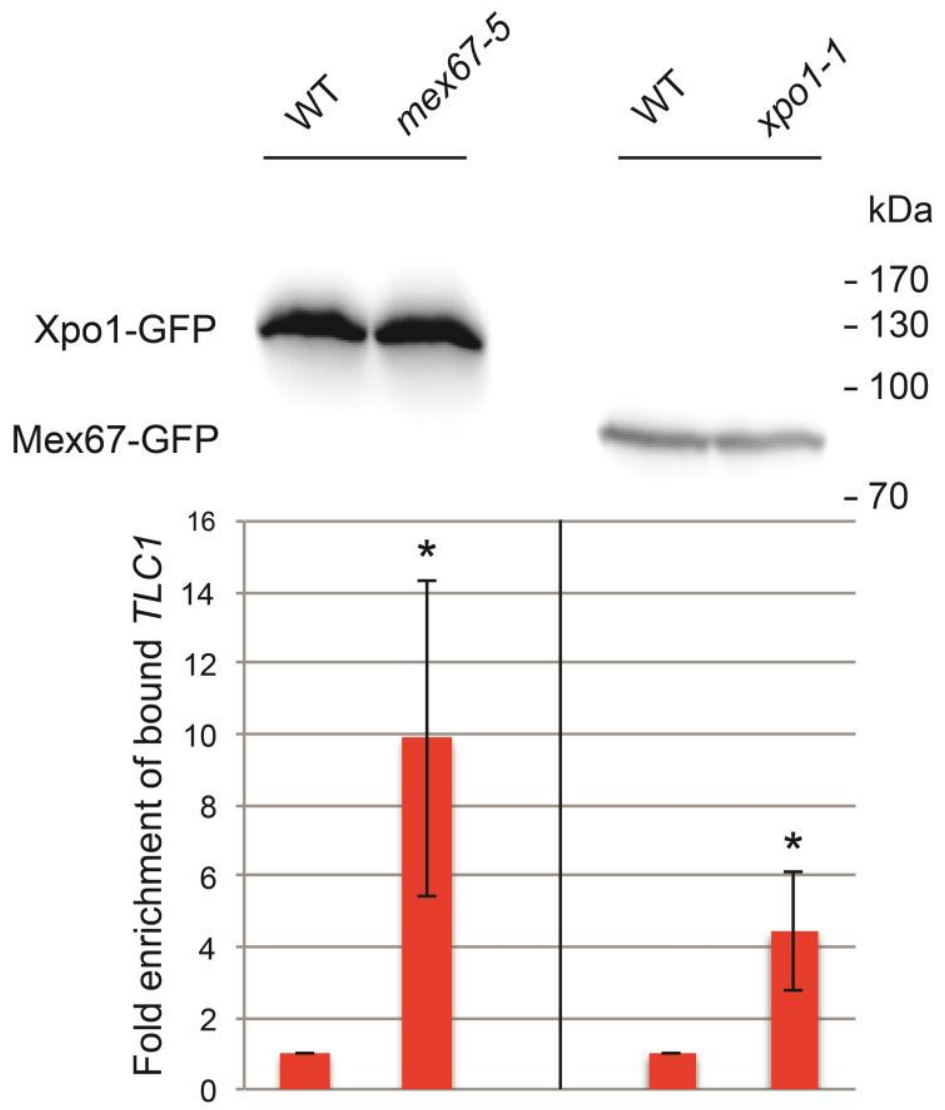

Figure 4.1.6 The TLC1 export activities of Xpo1/Crm1 and Mex67 are connected. Wild type (HKY381) and the mex67-5 (HKY644) cells were transformed with a XPO1-GFP containing plasmid (pCEN URA3 XPO1-GFP, pHK43). Additionally, wild type (HKY381) and the xpo1-1 (HKY206) cells were transformed with a MEX67-GFP containing plasmid (pCEN LEU2 pUN100-MEX67-GFP, pHK20). All strains (HKY380+pHK43, HKY644+pHK43, HKY381+pHK20, HKY206+pHK20) were grown to the logarithmic phase and shifted to $37^{\circ} \mathrm{C}$ for 1 hour. Xpo1-GFP and Mex67-GFP were immuno-precipitated by using anti-GFP antibodies and identified by using western blot analyses (top panel). The RNA bound to these proteins and the total RNA were extracted. RNA was used to quantify the TLC1 amount with the primer pair HK1385+HK1386 by qRT-PCR. The protein bound TLC1 was compared to the total TLC1 and these ratios were set into relation to that of wild type (mex67-5+pXPO1-GFP vs wild type+pXPO1-GFP, or xpo1-1+pMEX67-GFP vs wild type+pMEX67-GFP) (bottom panel). At least three independent experiments were done. Error bars indicate the standard deviation. P-value was calculated using two tailed, paired t-test $(*: 0.01 \leq p \leq 0.05)$. 
these two machineries function independently or coordinately, RNA co-immunoprecipitation experiments were performed. With the experiments, the TLC1 recruitment was analysed on either Mex67 or Xpo1 in the genetic background of mutating the genes of the opposite factors (mex67-5 + pXPO1-GFP or xpo1-1 + pMEX67-GFP) (figure 4.1.6).

Wild type (HKY381) and the mex67-5 (HKY644) cells were transformed with a XPO1-GFP containing plasmid (pXPO1-GFP, pHK43). Additionally, wild type (HKY381) and the xpo1-1 (HKY206) cells were transformed with a MEX67-GFP containing plasmid (pMEX67-GFP, pHK20). All strains (HKY380+pHK43, HKY644+pHK43, HKY381+pHK20, HKY206+pHK20) were grown to the logarithmic phase and shifted to $37^{\circ} \mathrm{C}$ for 1 hour. GFP tagged Xpo1 or Mex67 was precipitated by using anti-GFP antibodies. The precipitated proteins were identified through western blot analyses (figure 4.1.6 upper panel). The bound RNA was eluted from the precipitated proteins and the total RNA was extracted from the whole cell lysates. The RNA was used for qRT-PCR analyses and TLC1 was quantified from the RNA by using the primer pair HK1385+HK1386. To evaluate the interaction between the factors and $T L C 1$, the protein bound TLC1 was compared to the total TLC1 and the ratios were set into relation to that of wild type (mex67-5+pXPO1-GFP vs wild type+pXPO1-GFP, or xpo1-1+pMEX67-GFP vs wild type+ pMEX67-GFP).

The results (figure 4.1 .6 bottom panel) show an enriched TLC1 binding to one factor in the case of the defect of the other RNA transport factor. This observation suggests that Xpo1/Crm1 and Mex67 are connected through TLC1 and defect of one export factor will affect binding of the other one. Therefore, together with the fluorescent in situ hybridisation experiments showing that defects of either of these two pathways lead to a TLC1 export block (figure 4.1.1 and 4.1.2), the results suggest a cooperative manner of these two factors in transporting $T L C 1$.

Taken together, these results clearly indicate an important role of the mRNA export pathway in TLC1 nuclear export. Besides, both the mRNA export factor and Crm1/Xpo1 work cooperatively on this RNA translocation. Finally, it also suggests that although the nuclear export pathway is blocked in the export mutants, TLC1 appears to undergo maturation. 


\subsubsection{A TLC1 export block affects telomerase formation}

\subsubsection{TLC1 cytoplasmic deficiency leads to impaired localisation of the telomerase components}

The results shown above allow the conclusion that $T L C 1$ utilises the mRNA export pathway for its export. Further experiments were performed to uncover the effects of impaired TLC1 nuclear export on telomerase formation. Due to $T L C 1$ nucleo-cytoplasmic trafficking and the telomerase formation in the cytoplasm (Ferrezuelo et al., 2002; Gallardo et al., 2008), it can be proposed that the cytoplasmic presence of TLC1 would be important for formation and localisation of the telomerase complex. To directly address the effects of the TLC1 nuclear retention on the telomerase formation and localisation, immunofluorescence experiments were performed to track the localisation of the telomerase components, Est1 and Est2 (figure 4.1.7).

The immunofluorescence experiments were carried out in strains producing myc tagged Est1 and Est2 in the background of wild type, xpo1-1 or the mRNA export defective mutants (figure 4.1.7A). The strains EST1-12xMYC 3xFLAG-12xMYC-EST2 (wild type, HKY1292), EST1-12xMYC 3xFLAG-12xMYC-EST2 t/c1::HIS (t/c14, HKY1294), EST1-12xMYC 3xFLAG12xMYC-EST2 xpo1::LEU2+pxpo1-1 (xpo1-1, HKY 1302), EST1-12xMYC 3xFLAG-12xMYC-EST2 rat7-1 (rat7-1, HKY1332), EST1-12xMYC 3xFLAG-12xMYC-EST2 rat8-2 (rat8-2, HKY1334) and EST1-12xMYC 3xFLAG-12xMYC-EST2 mex67::HIS+pmex67-5 (mex67-5, HKY1336) were grown to the logarithmic phase. Half of the cells were kept at $25^{\circ} \mathrm{C}$ and the other half were shifted to $37^{\circ} \mathrm{C}$ for 1 hour. The localisations of Est1 and Est2 in the cells were detected by using mouse anti-myc antibodies and AlexaFluor488 conjugated goat anti-mouse secondary antibodies.

The results (figure 4.1.7A) show a nuclear enrichment of Est1 and Est2 in wild type cells, representing the nuclear localisation of the telomerase components. In contrast, Est1 and Est2 are mislocalised to the cytoplasm in the absence of $T L C 1$, indicating a failure of the telomerase assembly. Similarly, in the TLC1 export defective mutant, xpo1-1, a significant 
reduction of nuclear Est1 and Est2 distribution was observed under the non-permissive condition $\left(37^{\circ} \mathrm{C}\right)$. Although there was nuclear signal remaining, it can be explained by the presence of the pre-existing telomerase. Similar to xpo1-1, the mRNA export defective mutants, mex67-5, rat7-1 and rat8-2, also show a comparable distribution of the telomerase components in the cytosol compared to wild type at the non-permissive condition $\left(37^{\circ} \mathrm{C}\right)$.

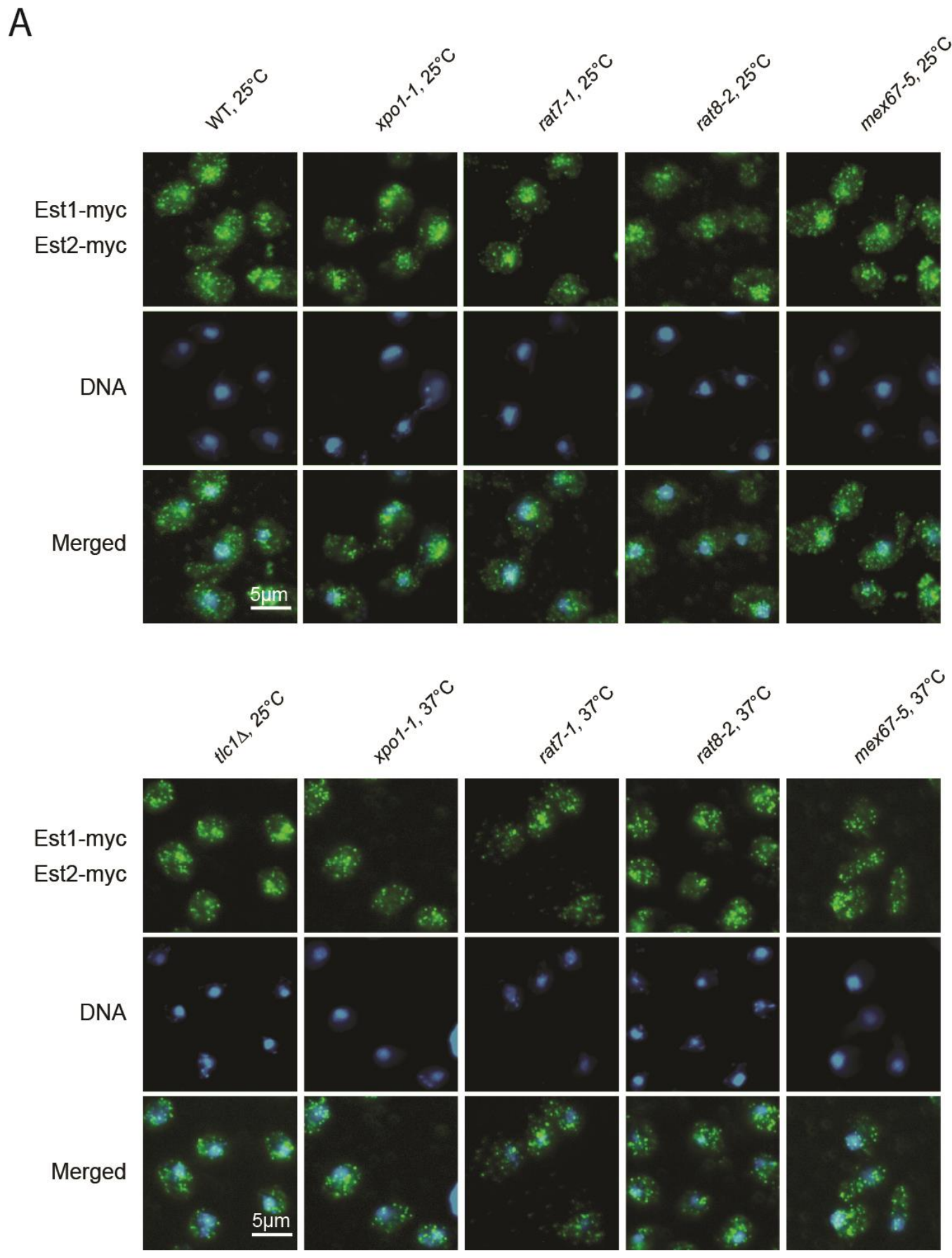

Figure legend see next page 


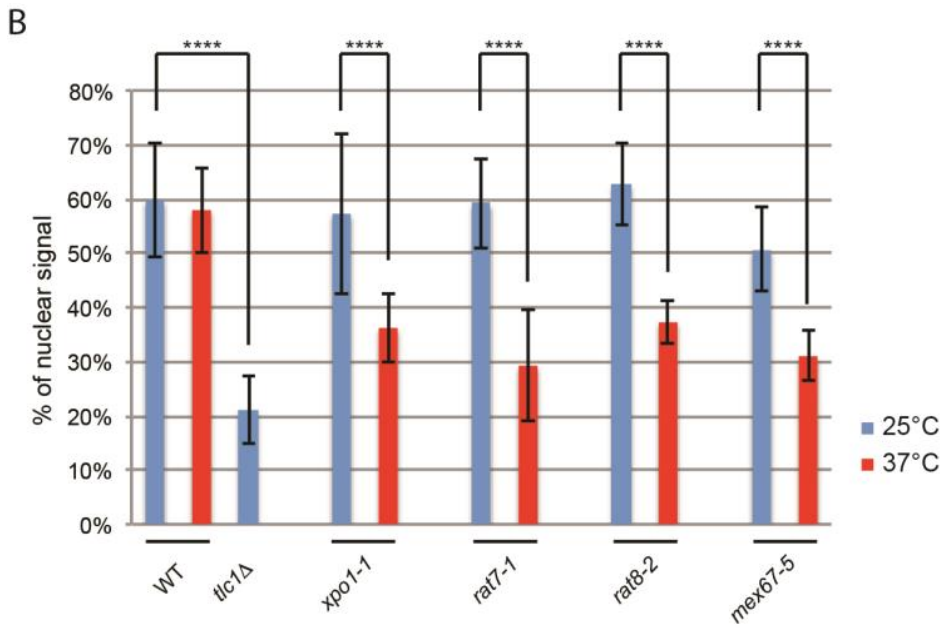

Figure 4.1.7 Telomerase components localisation is impaired due to TLC1 export defects. All indicated strains were grown to the logarithmic phase. The cells were split into two equal portions. One portion was kept at $25^{\circ} \mathrm{C}$ and the other portion was shifted to $37^{\circ} \mathrm{C}$ for 1 hour. (A) Myc tagged Est1 and Est2 were detected by using mouse anti-myc antibodies and AlexaFluor488 anti-mouse antibodies (green). The nuclei were stained by hoechst33342 (blue). Scale bars: $5 \mu \mathrm{m}$. (B) The ratios of the nuclear signal to total cell signal were shown in the diagram. For each condition the ratios of 20 cells were calculated. At least three independent experiments were done, one of which is shown in figure A. Error bars indicate the standard deviation. P-value was calculated using two-tailed, unpaired t-test $(* * * *: p<0.0001)$

This suggests that due to the TLC1 cytoplasmic deficiency in these mutants, the Est proteins are not imported into the nucleus. Signal quantification (figure 4.1.7B) reveals the change of the telomerase component localisation through calculating the ratio between the nuclear and total signal ( $\mathrm{N} / \mathrm{T}$ ratio) (figure 4.1.7B). Compared to the wild type ( $60 \% \mathrm{~N} / \mathrm{T}$ ) and t/c1 $\Delta$ $(\sim 21 \% \mathrm{~N} / \mathrm{T})$ strains, mex67-5 ( 29\% N/T), rat7-1 ( 37\% N/T) and rat8-2 ( 30\% N/T) mutants show similar N/T ratios to xpo1-1 $(\sim 36 \% \mathrm{~N} / \mathrm{T})$ at $37^{\circ} \mathrm{C}$. Furthermore, comparing $25^{\circ} \mathrm{C}$ to $37^{\circ} \mathrm{C}$ in a given strain, the export mutants show an obvious reduction of the nuclear localisation of the telomerase components, e.g. xpo1-1 ( 21\% N/T ratio reduction), rat7-1 ( $\sim 30 \% \mathrm{~N} / \mathrm{T}$ ratio reduction), rat8-2 ( $\sim 26 \% \mathrm{~N} / \mathrm{T}$ ratio reduction), and mex67-5 ( $\sim 20 \% \mathrm{~N} / \mathrm{T}$ ratio reduction). Summed up, these results suggest that (1) Like Xpo1, the mRNA export factors also participate in transporting $T L C 1$; $(2)$ the model that the telomerase might be formed in the cytoplasm following the TLC1 nuclear export is further supported; (3) the conclusion that Est1 nuclear re-import is independent of TLC1 transport (Hawkins and Friedman, 2014) is however challenged. 
Since Est1 and Est2 are mislocalised to the cytoplasm due to the TLC1 nuclear export block in the mRNA export mutants, the amount of Est1 and Est2 in the cytoplasm might also be increased in this case. To obtain further evidence, cytoplasmic fractionation experiments were performed (figure 4.1.8).

Similar to the cell fractionation experiments in section 4.1.1.2, the strains mentioned above were fractionated and their cytoplasmic fractions were extracted. Zwf1 and Nop1, which were mentioned in section 4.1.1.2, were used as indicators to verify the fractionation experiments. As shown in the total cell lysates ( $T$ in figure 4.1.8), all Est1, Est2, Nop1 and Zwf1 were detectable; however, in the cytoplasmic fractions (C in figure 4.1.8), Nop1 was not detectable, suggesting the successful elimination of the nuclear fraction. Subsequently, the cytoplasmic amount of Es1+Est2 at $37^{\circ} \mathrm{C}$ was compared to that at $25^{\circ} \mathrm{C}$ and the ratios were set into relation to that of the wild type at $25^{\circ} \mathrm{C}$ (figure 4.1 .8 bottom panel). In addition, the amount of the cytoplasmic Est1+Est2 in $t / c 1 \Delta$ at $25^{\circ} \mathrm{C}$ was also compared to that in wild type and the ratios were used as positive controls.

The experiments (figure 4.1.8) clearly show a cytoplasmic retention of the telomerase components once $T L C 1$ is unable to reach the cytoplasm to serve as the scaffold for the telomerase formation. The quantification indicates a dramatic cytoplasmic mislocalisation of the telomerase components in the absence of TLC1 ( 8 fold cytoplasmic enrichment comparing t/c1 $\Delta$ to wild type). Moreover, in the TLC1 nuclear export defective mutants, xpo1-1 ( 4 fold cytoplasmic enrichment comparing $37^{\circ} \mathrm{C}$ to $\left.25^{\circ} \mathrm{C}\right)$, mex67-5 $(\sim 3$ fold cytoplasmic enrichment comparing $37^{\circ} \mathrm{C}$ to $\left.25^{\circ} \mathrm{C}\right)$ as well as rat8-2 ( 4 fold cytoplasmic enrichment comparing $37^{\circ} \mathrm{C}$ to $25^{\circ} \mathrm{C}$ ), show a comparable mislocalisation phenotype of the telomerase components, suggesting that the nuclear re-import of the telomerase components is dependent on TLC1 nuclear export. 


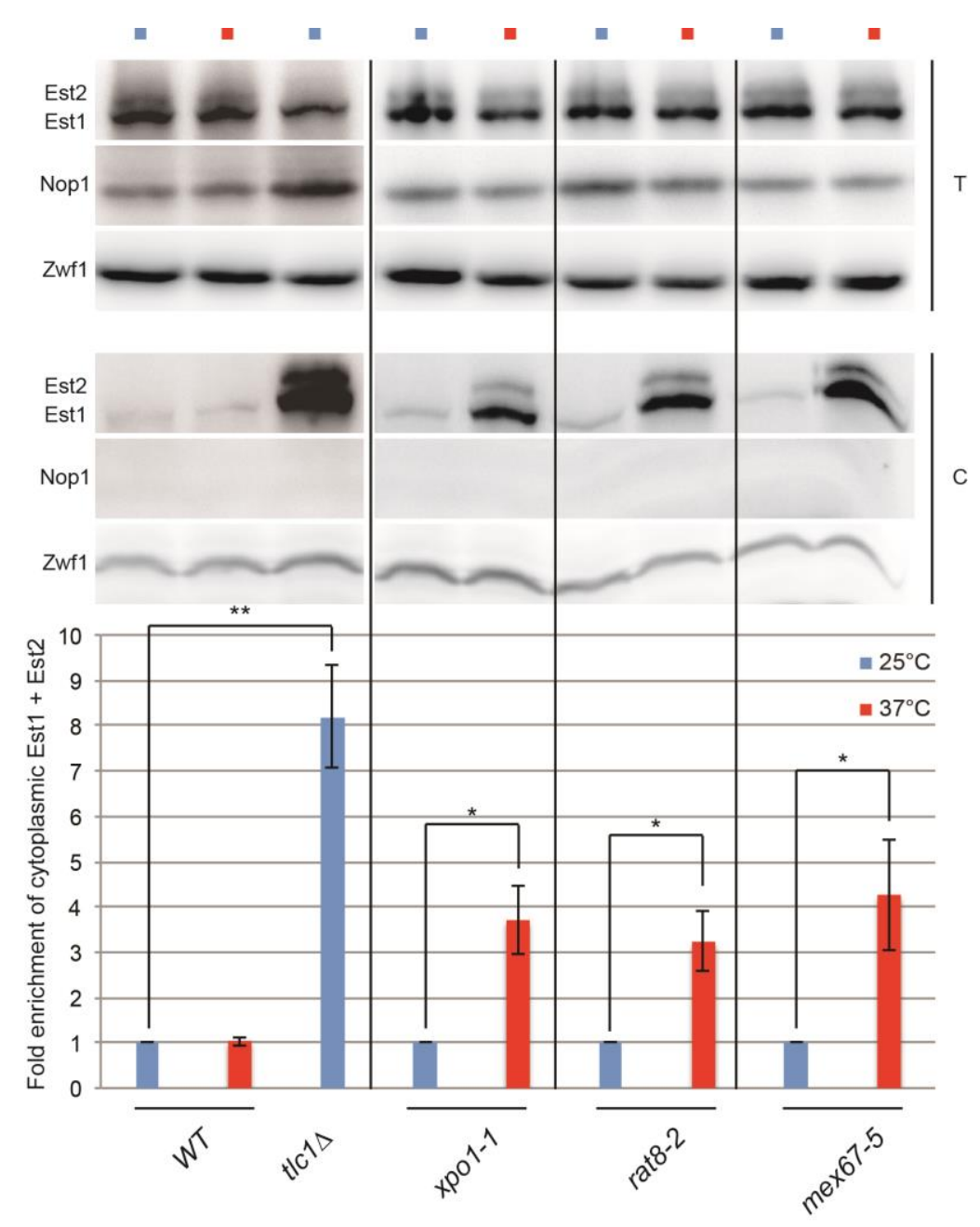

Figure 4.1.8 TLC1 cytoplasmic deficiency leads to the cytoplasmic enrichment of the telomerase components. All indicated strains were grown to the logarithmic phase. Half of the cells were kept at $25^{\circ} \mathrm{C}$ and the other half were shifted to $37^{\circ} \mathrm{C}$ for 1 hour. Cells were fractionated and the cytoplasmic fractions were isolated. The total cell extracts $(\mathrm{T})$ and the cytoplasmic fractions $(\mathrm{C})$ were analysed through western blot analyses (upper panel). Est1-myc and Est2-myc were detected using anti-myc antibodies. Zwf1 and Nop1 were used as the cytoplasmic and nuclear indicators and were detected using rabbit anti-Zwf1 and anti-Nop1 antibodies, respectively. The amount of Est1+Est2 at $37^{\circ} \mathrm{C}$ was compared to that at $25^{\circ} \mathrm{C}$. In addition, the amount of the cytoplasmic Est1 and Est2 in t/c1 4 was compared to that in wild type. The ratios are shown in the diagram (lower panel). At least three independent experiments were done, one of which is shown in the upper panel. Error bars indicate the standard deviation. P-value was calculated using two-tailed, paired t-test $(*: 0.01 \leq p \leq 0.05$;**: $0.001 \leq p \leq 0.01$ ). 


\subsubsection{Less TLC1-Est2 interactions are formed due to the TLC1 nuclear retention}

Since the results above show a mislocalisation of the telomerase components, which could be due to the reduction of the cytoplasmic presence of $T L C 1$, a reduced physical interaction between TLC1 and the telomerase components would be expected. RNA co-immunoprecipitation experiments were performed to determine the association of TLC1 to the telomerase component in the cytoplasm (figure 4.1.9).

The strains (HKY1292, HKY1302, HKY1332, HKY1334 and HKY1336) that were used in section 4.1.2.1 were also used in these experiments. The cells were grown to the logarithmic phase at $25^{\circ} \mathrm{C}$. The cells were either retained at $25^{\circ} \mathrm{C}$ or shifted to $37^{\circ} \mathrm{C}$ for 1 hour. Est2 was precipitated by using anti-FLAG antibodies. The Est2 bound RNA was subsequently eluted from the precipitated proteins and the total RNA was extracted from the whole cell lysates. TLC1 was quantified through QRT-PCR by using the specific TLC1 primer pair (HK1385+HK1386). The Est2 bound TLC1 in a given strain was first compared to the total $T L C 1$. This ratios at $37^{\circ} \mathrm{C}$ was further compared to that at $25^{\circ} \mathrm{C}$ in this strain. The ratios were finally set into relation to the wild type.

The results show that the interactions between TLC1 and Est2 are reduced not only in xpo1-1 ( $\sim 34 \%$ reduced), but also in the mRNA export mutants ( $\sim 36 \%$ in rat $7-1, \sim 33 \%$ in rat8-2 and $\sim 41 \%$ in mex67-5). These results support the potential role of mRNA export factors in TLC1 nuclear export and the model of cytoplasmic assembly of the telomerase complex (Ferrezuelo et al., 2002). 


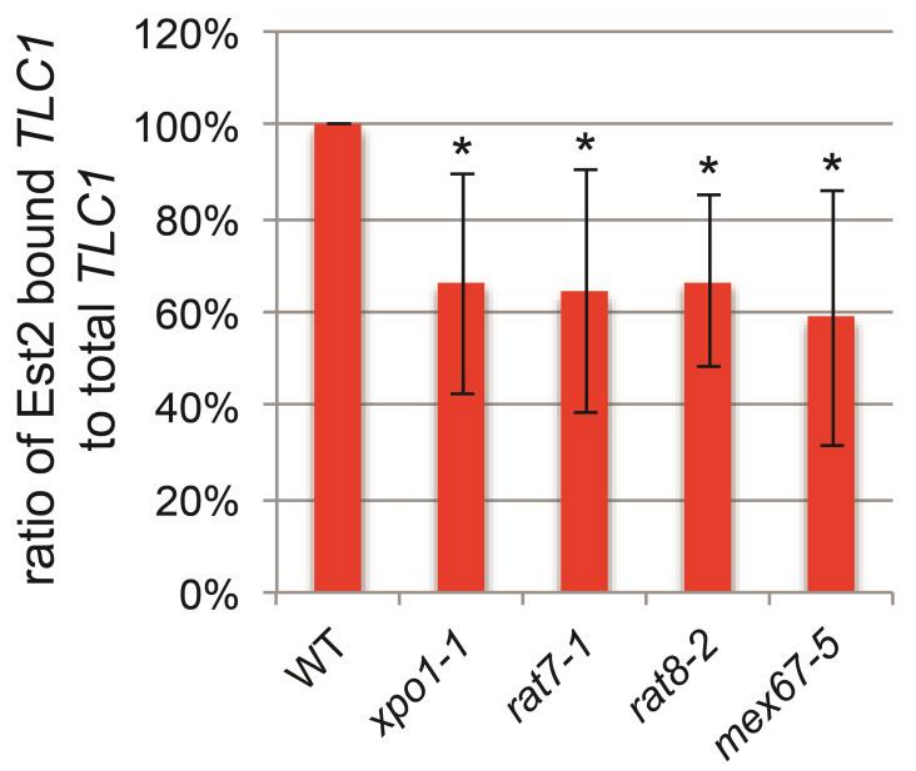

Figure 4.1.9 RNA co-immunoprecipitation experiments show a reduced association of TLC1 to Est2 in the export defective mutants. All indicated strains were grown to the logarithmic phase. Half of the cells were kept at $25^{\circ} \mathrm{C}$ and the other half were shifted to $37^{\circ} \mathrm{C}$ for 1 hour. FLAG tagged Est2 was precipitated by using mouse anti-FLAG antibodies. RNA bound to Est2 the total RNA was extracted. TLC1 was quantified through qRT-PCR by using the specific TLC1 primer pair (HK1385+HK1386). The bound TLC1 was compared to the total TLC1. This ratio at $37^{\circ} \mathrm{C}$ was further compared to that at $25^{\circ} \mathrm{C}$. They were finally set into relation with that of wild type. At least three independent experiments were done. Error bars indicate the standard deviation. The $p$-value was calculated using two-tailed, paired t-test $\left({ }^{*}: 0.01 \leq p \leq 0.05\right)$. 


\subsubsection{Reduction of the TLC1 cytoplasmic presence affects the interaction between the telomerase components}

The interaction between Est1 and Est2 is indirect and mediated by TLC1 (Lubin et al., 2012). Due to the scaffold function of TLC1 on composition of the telomerase complex, the components are not able to be assembled to build up the telomerase complex without TLC1. Therefore, since it has been concluded that telomerase formation might occur in the cytoplasm and this constitution needs cytoplasmic TLC1 presence, the decreased TLC1 nuclear export might reduce the efficiency of telomerase assembly. Co-immunoprecipitation experiments were performed to examine the interaction between telomerase components, Est1 and Est2, to identify the telomerase assembly (figure 4.1.10).

The strains (HKY1292, HKY1294, HKY1302, HKY1332, HKY1334 and HKY1336) that were used in section 4.1.2.1 were also used in these experiments. All strains were grown to the logarithmic phase and were either retained at $25^{\circ} \mathrm{C}$ or shifted to $37^{\circ} \mathrm{C}$ for 1 hour. FLAG tagged Est2 was precipitated with anti-FLAG antibodies. In addition, one of the wild type sample was treated with RNaseA (final concentration: $0.1 \mathrm{mg} / \mathrm{ml}$ ) to remove $T L C 1$ and its mediated Est1-Est2 interaction. Both Est1 and Est2 were detected by using the anti-myc antibodies through western blot analyses. Besides, the total cell lysates were used as input controls and Hem15, a mitochondrial inner membrane protein, was used as loading control. Hem15 was detected by anti-Hem15 antibodies. The interactions between Est1 and Est2 were evaluated through calculating the ratios of the amount of Est2 to Est1. These ratios at $37^{\circ} \mathrm{C}$ were further set into relation to those at $25^{\circ} \mathrm{C}$ in a given strain. The protein levels of Est1 and Est2 were evaluated through comparing the amount of Est1+Est2 from the input control to that of Hem15 from the loading control. These ratios were also set into relation to that of wild type. Apparently, decreased interactions of the telomerase components are observed in the TLC1 export mutants (figure 4.1.10A). The quantification of the interactions (figure 4.1.10B) shows that $\sim 89 \%$ of the interaction between Est1 and Est2 is reduced if $T L C 1$ is not present in $t / c 1 \Delta$ strains. In wild type cells upon RNase treatment, $\sim 65 \%$ of the interaction is also reduced. Since Est1 and Est2 interact through $T L C 1$, incomplete destruction of Est1-Est2 interaction 
from this RNase treatment might be probably due to the compact clustering of the telomerase complex reported previously (Gallardo et al., 2011). Interestingly, the TLC1 export defective mutants show only $\sim 53 \%, \sim 58 \%, \sim 64 \%$ and $\sim 67 \%$ of the Est1-Est2 interactions remaining in xpo1-1, rat7-1, rat8-2 and mex67-5, respectively. In addition, the protein levels of Est1 and Est2 were also quantified to identify the effect of the RNA export defects on gene expression (figure 4.1.10C). Interestingly, stronger defect on producing of the telomerase components, especially Est2, is found in $t / c 1 \Delta$ and this is coincident to previous findings showing only 50\% EST2 expression level in the t/c1 $\Delta$ strain (Taggart et al.,

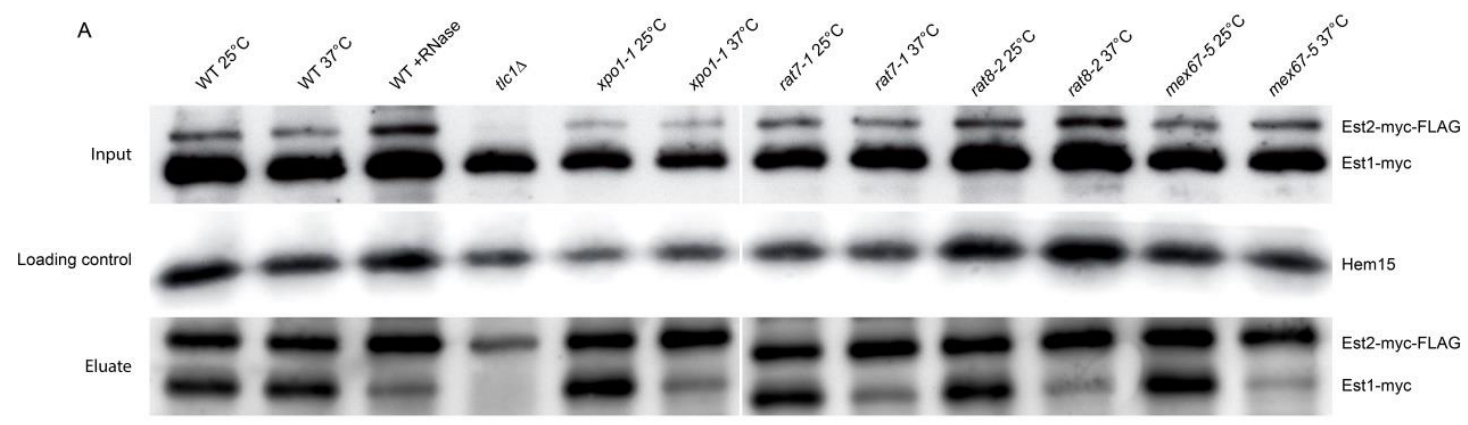

B

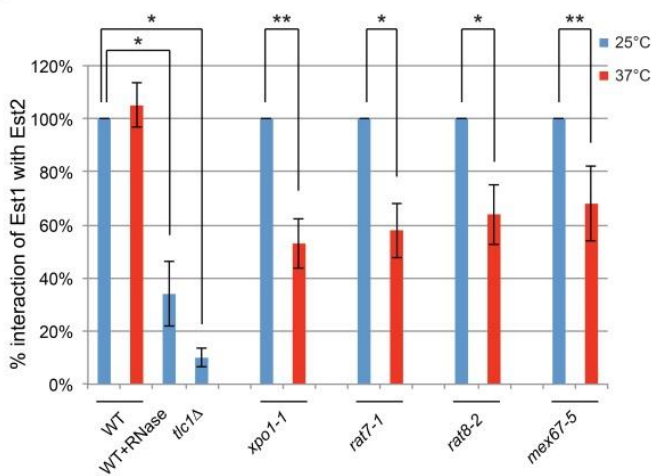

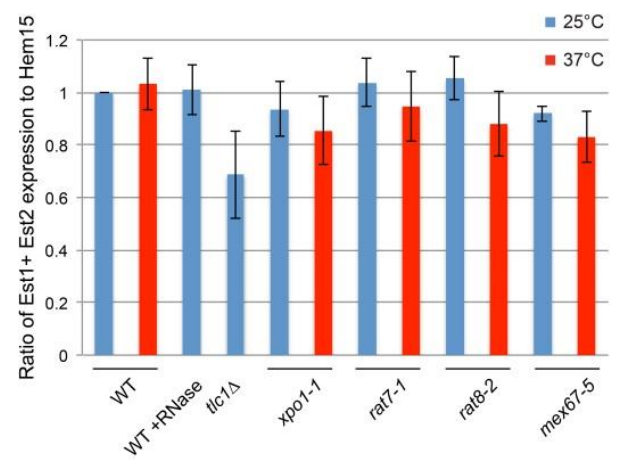

Figure 4.1.10 The interactions between Est1 and Est2 are impaired in the export mutants. All indicated strains were grown to the logarithmic phase. Half of the cells were kept at $25^{\circ} \mathrm{C}$ and the other half were shifted to $37^{\circ} \mathrm{C}$ for 1 hour. One additional portion of the wild type sample was treated with RNaseA (final concentration: $0.1 \mathrm{mg} / \mathrm{ml}$ ). To precipitate FLAG tagged Est2, mouse anti-FLAG antibodies were used. (A) Precipitated Est2 and its interacted Est1 were detected through western blot analyses by using rabbit anti-myc antibodies. The whole cell lysates were used as input controls. Hem15 was used as loading control and was detected by using anti-Hem15 antibodies. (B) The Est1-Est2 interactions were evaluated through calculating the ratios between the amount of Est2 and Est1. The ratios at $37^{\circ} \mathrm{C}$ were further set into relation to that at $25^{\circ} \mathrm{C}$. The P-value is calculated using two-tailed, paired t-test $\left({ }^{*}: 0.01 \leq p \leq 0.05 ;{ }^{* *}: 0.001 \leq p \leq 0.01\right)$. (C) The protein levels of the telomerase components were reflected by calculating the ratios of the total amount of Est1 and Est2 to Hem15. Error bars indicate the standard deviation. At least three independent experiments were done, one of which is shown in figure $A$. 
2002). Why the expression level of telomerase components is dependent on TLC1 is still poorly understood and is supposed to be a consequence of an accelerated degradation of the unbound telomerase components.

Furthermore, since the Ku-complex is related to TLC1 nuclear import (Gallardo et al., 2008) and to the recruitment of the telomerase onto telomeres (Fisher et al., 2004), the improper TLC1 localisation, which leads to a defect in telomerase formation, is proposed to reduce the binding of the telomerase components to the Ku components. This hypothesis was tested by performing co-immunoprecipitation experiments to identify the interaction between Est2 and Yku70 (figure 4.1.11).

All strains (HKY1292, HKY1294, HKY1302, HKY1332, HKY1334 and HKY1336) used above were grown to the logarithmic phase and were either retained at $25^{\circ} \mathrm{C}$ or shifted to $37^{\circ} \mathrm{C}$ for 1 hour. Est2 was precipitated by using anti-FLAG antibodies and Yku70 was detected through western blot analyses by using anti-Yku70 antibodies. Additionally, the total cell lysates were used as input controls; Zwf1 was used as loading control and was detected by using anti-Zwf1 antibodies. The ratio of Yku70 to Est2 at $37^{\circ} \mathrm{C}$ were primarily compared to that at $25^{\circ} \mathrm{C}$ and subsequently set into relation with that of wild type.

The result shows that, in the case of no TLC1 export defect, the interaction of Est2 with Yku70 is similar in the export defective mutants and the wild type at $25^{\circ} \mathrm{C}$. However, this interaction is reduced upon a temperature shift ( $\sim 60 \%$ in $x p 01-1, \sim 41 \%$ in rat8-2 and $\sim 59 \%$ in mex67-5, respectively) suggesting that the TLC1 cytoplasmic deficiency leads to an impairment of the telomerase assembly in xpo1-1 and the mRNA export mutants. 


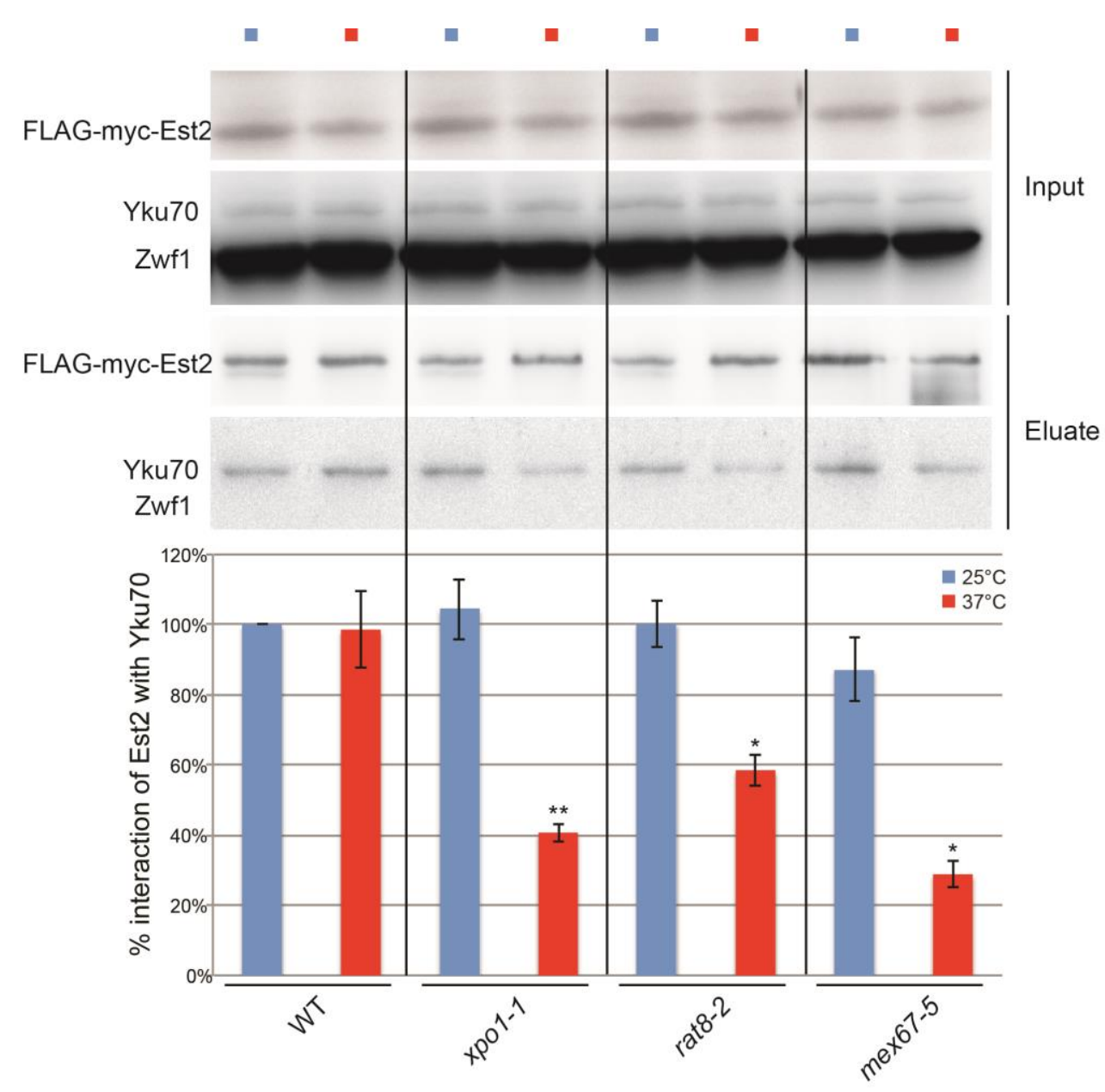

Figure 4.1.11 The interactions between Est2 and Yku70 are reduced due to the TLC1 nuclear export block. All indicated strains were grown to the logarithmic phase and were either retained at $25^{\circ} \mathrm{C}$ or shifted to $37^{\circ} \mathrm{C}$ for 1 hour. Est2 was precipitated through mouse anti-FLAG antibodies. Est2 and its interacted Yku70 were analysed via western blot analyses (upper panel). Est2 was detected by anti-FLAG antibodies and Yku70 was detected by anti-Yku70 antibodies. Total cell lysates were used as input controls. Zwf1 was detected by anti-Zwf1 antibodies and was used as the loading control. The amount of Est2 was compared to that of Yku70. These ratios were set into relation to that of the wild type and were shown in the diagram (lower panel). At least three independent experiments were done, one of which is shown in the upper panel. The error bars indicate the standard deviation. P-value was calculated using two-tailed, paired t-test $\left({ }^{*}: 0.01 \leq \mathrm{p} \leq 0.05 ;{ }^{* *}: 0.001 \leq \mathrm{p} \leq 0.01\right)$ 


\subsubsection{Analyses of the mex67-5 xpo1-1 double mutant on TLC1 nuclear export}

\subsubsection{The mex67-5 xpo1-1 double mutant shows a stronger TLC1 nuclear accumulation and an increased rate of TLC1 processing}

Since both of $\mathrm{Crm} 1 / \mathrm{Xpo1}$ and Mex67 were identified to mediate TLC1 nuclear export, an enhancement of the TLC1 nuclear export block would be expected if both pathways were simultaneously mutated. Fluorescent in situ hybridisation experiments were performed to identify the localisation of TLC1 in the mex67-5 xpo1-1 double mutant (figure 4.1.12). In addition, to distinguish the export block from an increased nuclear import of TLC1, the $y k u 70 \Delta$ background mentioned in section 4.1.1.1 was also used in these experiments.

All strains wild type (HKY381), yku704 (HKY1073), xpo1-1 yku704 (HKY1444), mex67-5 yku70 $\Delta$ (HKY1397) and xpo1-1 mex67-5 yku70 4 (HKY1445) were grown to the logarithmic phase at $25^{\circ} \mathrm{C}$ before they were shifted to $37^{\circ} \mathrm{C}$ for 1 hour. $T L C 1$ was detected by using Cy3 labelled specific TLC1 oligo probe (HK1761, HK1789 and HK1790). The nuclear/total signal ratios $(\mathrm{N} / \mathrm{T})$ were calculated through comparing the nuclear signals to the total cellular signals of TLC1 and the ratios were subsequently set into relation to that of $y k u 70 \Delta$.

Strikingly, in the double mutant an increased level of TLC1 nuclear accumulation ( 2.4 fold $\mathrm{N} / \mathrm{T}$ signal ratio compared to $y k u 70 \Delta$ ) is observed in contrast to the mutant containing any defective single pathway $(\sim 1.8$ fold $\mathrm{N} / \mathrm{T}$ signal ratio compared to $y k u 70 \Delta$ in $x p o 1-1$ and $\sim 1.9$ fold N/T signal ratio compared to $y k u 70 \Delta$ in mex67-5) (figure 4.1.12). This result suggests a combined action of both factors on transporting TLC1.

Furthermore, as mentioned in 4.1.1.4, although the TLC1 nuclear export block does not alter the procedure of the TLC1 processing, it changes the rate of the processing of $T L C 1$. In the TLC1 export mutants, more TLC1 undergoes processing than in wild type. Therefore, an even higher rate of the processing of TLC1 would be expected in the double mutant xpo1-1 


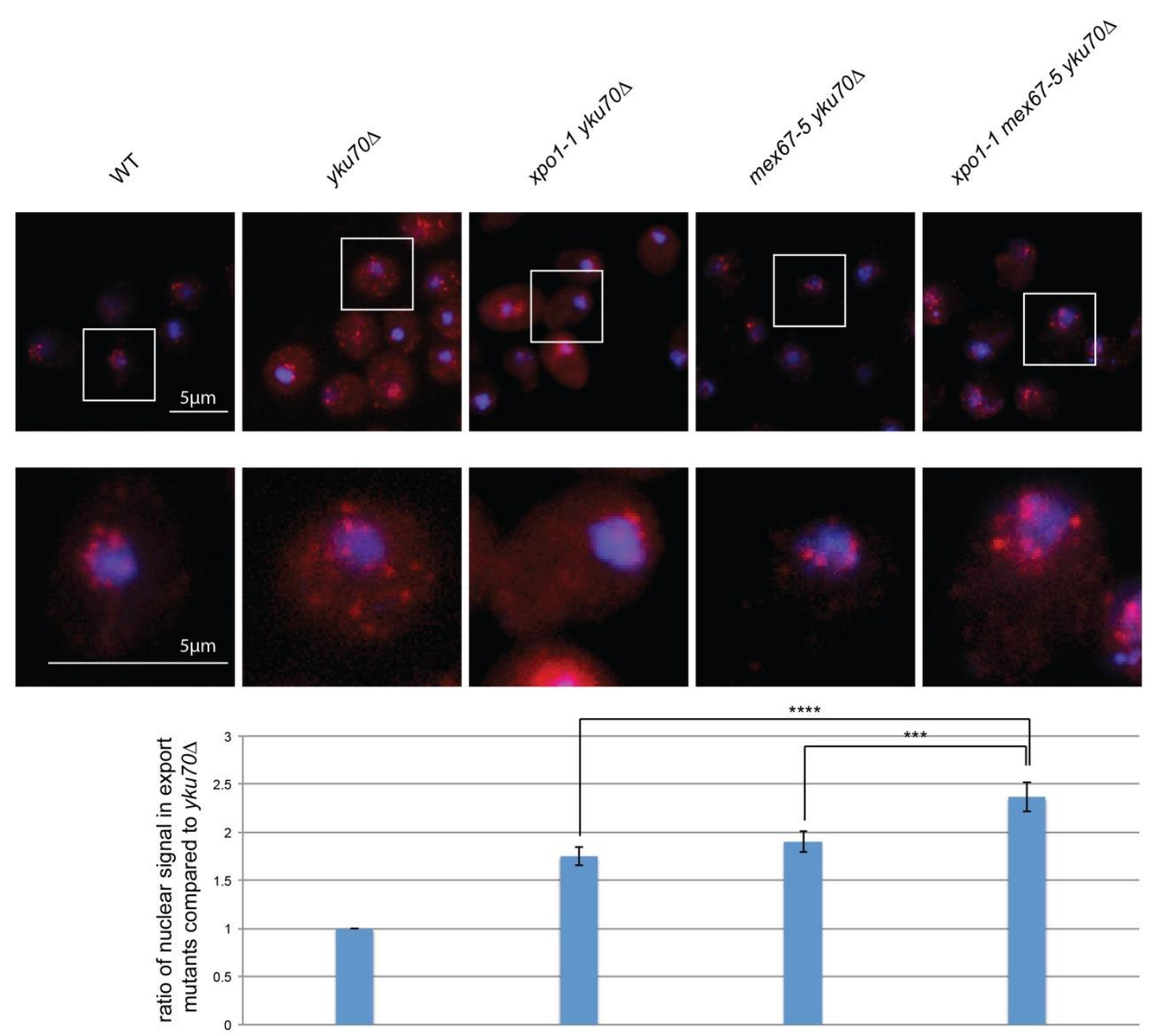

Figure 4.1.12 Fluorescent in situ hybridisation experiments show an increased nuclear mislocalisation of TLC1 in the double mutant xpo1-1 mex67-5. The indicated strains were grown to the logarithmic phase and were shifted to $37^{\circ} \mathrm{C}$ for 1 hour. $T L C 1$ was detected by specific Cy3 labeled oligo TLC1 probes (HK1761, HK1789, HK1790) (red) (top panel). The nuclei were stained with hoechst33342 (blue). The ratios between nuclear TLC1 signal and total TLC1 signal were calculated. The ratios were set into relation to that of $y k u 70 \Delta$ and were showed in the diagram (bottom panel). At least 20 cells were quantified for each condition. At least three independent experiments were done, one of which is shown in the upper panel. Error bars indicate the standard deviation. The P-value was calculated using two-tailed, unpaired t-test $\left({ }^{* * *}: 0.0001 \leq \mathrm{p} \leq 0.001 ;{ }^{* * * *}\right.$ : $\mathrm{p} \leq 0.0001)$.

mex67-5 than in the single mutants. To test this, qRT-PCR analyses were performed to examine the effect of the double mutant xpo1-1 mex67-5 on the processing of TLC1 (figure 4.1.13).

All strains, wild type (HKY381), xpo1-1 (HKY206), mex67-5 (HKY644) and xpo1-1 mex67-5 (HKY1353) were grown to the logarithmic phase and shifted to $37^{\circ} \mathrm{C}$ for 1 hour. RNA was extracted from the whole cell lysates. The total TLC1 and the unprocessed TLC1 were 
quantified by qRT-PCR with specific primer pairs HK1385+HK1386 and HK1382+HK1384 as mentioned in section 4.1.1.4. Furthermore, as the reference, the U6 snRNAs were quantified by using the primer pair HK1404+HK1405. To evaluate the transcription levels of the total TLC1, the amount of the total TLC1 was compared to that of the U6 SNRNA and the ratios were set into relation to that of the wild type (figure 4.1.13A). The processing of TLC1 was evaluated by comparing the amount of the unprocessed TLC1 to that of the total TLC1 and the ratios were also set into relation to that of wild type (figure 4.1.13B).

The results (figure 4.1.13) indicate that in the mex67-5 xpo1-1 double mutant, the amount of the total $T L C 1$ was slightly reduced ( $\sim 82 \%$ of the wild type amount). Nevertheless, the ratio of the unprocessed TLC1 to the total TLC1 is dramatically reduced ( 3\% of the wild type level) in contrast to any one of the single mutants ( $17 \%$ in xpo1-1 and $\sim 30 \%$ in mex $67-5$, of the wild type level) (figure 4.1.13B). Considering that in wild type only $5-10 \%$ of the unprocessed $T L C 1$ exists in the total $T L C 1, \sim 3 \%$ unprocessed $T L C 1$ of the wild type level in xpo1-1 mex67-5 indicates that the vast majority of $T L C 1$ (more than 99\%) undergoes processing in the xpo1-1 mex67-5 double mutants. These results point out that the xpo1-1 mex67-5 double mutant shows a similar but even stronger phenotype on TLC1 processing than the single mutants, xpo1-1 or mex67-5. These results also suggest that the processing of TLC1 occurs prior to its nuclear export. 
A
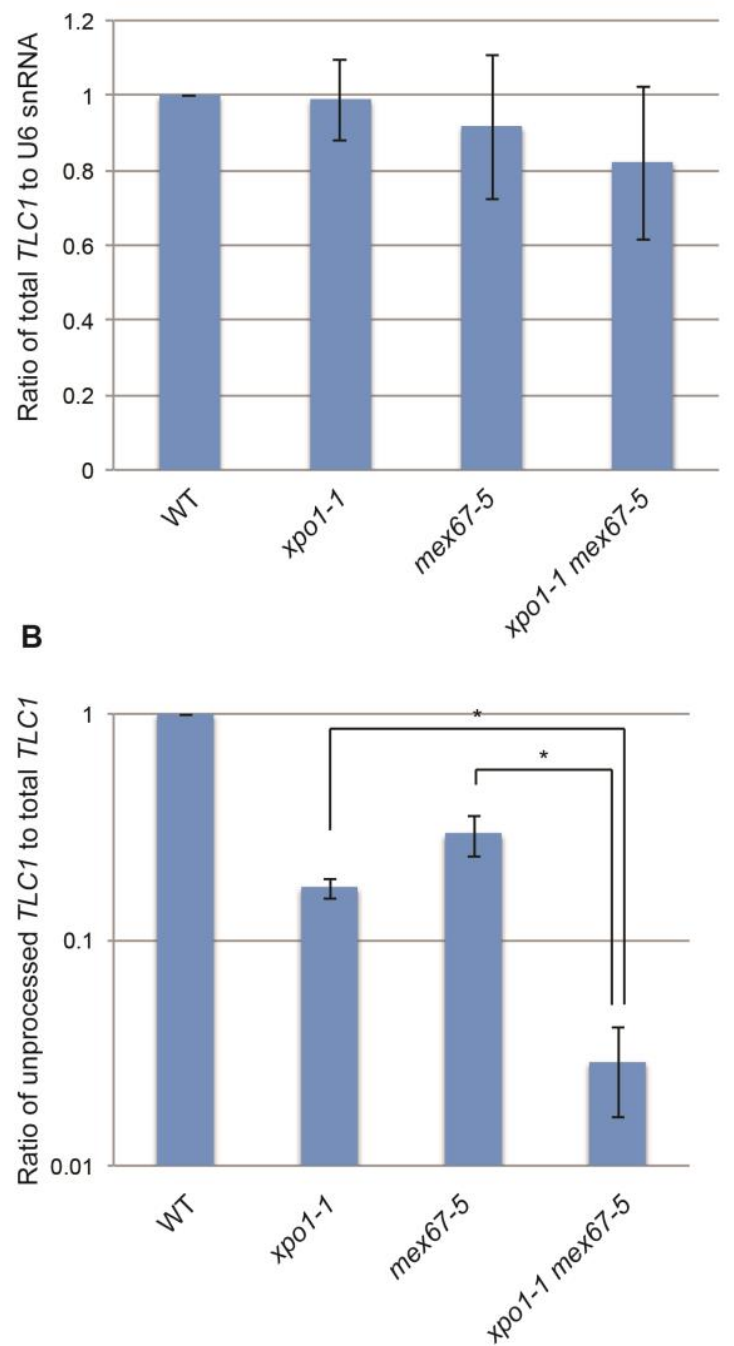

Figure 4.1.13 The xpo1-1 mex67-5 double mutant shows a higher TLC1 processing rate than the single mutants, xpo1-1 and mex67-5. All indicated strains were grown to the logarithmic phase and shifted to $37^{\circ} \mathrm{C}$ for 1 hour. The total $T L C 1$ and the unprocessed $T L C 1$ were quantified with specific primer pairs (HK1385+HK1386 for total TLC1, HK1382+HK1384 for unprocessed TLC1) through qRT-PCR. The U6 snRNA was quantified as reference by using the primer pair HK1404+HK1405. (A) The transcription levels of the total TLC1 were calculated through comparing the amount of the total TLC1 to that of the U6 snRNA. The ratios were set into relation to that of the wild type. (B) The TLC1 processing rates were calculated through comparing the amount of the unprocessed TLC1 to that of the total $T L C 1$. The ratios were set into relation to that of the wild type. At least three independent experiments were done. Error bars indicate the standard deviation. P-value was calculated using two-tailed, paired t-test $(*: 0.01 \leq p \leq 0.05)$ 


\subsubsection{The cytoplasmic mislocalisation of a telomerase component is increased in the xpo1-1 mex67-5 double mutant}

Since TLC1 export defect leads to cytoplasmic mislocalisation of the telomerase components, a stronger mislocalisation would be expected in the xpo1-1 mex67-5 double mutant. To test this hypothesis, immunofluorescence experiments were performed to examine the localisation of the telomerase component, Est1 (figure 4.1.14).

All strains EST1-3xMYC (wild type, HKY1463), EST1-3xMYC xpo1-1 (HKY1464), EST1-3xMYC mex67-5 (HKY1465) and EST1-3xMYC xpo1-1 mex67-5 (HKY1466) were grown to the logarithmic phase and were subsequently either retained at $25^{\circ} \mathrm{C}$ or shifted to $37^{\circ} \mathrm{C}$ for 1 hour. Myc tagged Est1 was detected by mouse anti-myc antibodies and sheep anti-mouse-AlexFluor488 secondary antibodies. In addition to the immunofluorescence experiments, the cells were imaged through 6-10 z-stacks $(0.2 \mu \mathrm{m} / \mathrm{stack})$ followed by deconvolution to acquire sharper and more focussed images .

The results show that the Est1 proteins are localised to the nucleus at $25^{\circ} \mathrm{C}$ and $37^{\circ} \mathrm{C}$ in the wild type. In contrast to that, in xpo1-1, mex67-5 as well as the xpo1-1 mex67-5 double mutant, the Est1 proteins are distributed in the cytoplasm upon a temperature shift to $37^{\circ} \mathrm{C}$. Furthermore, in contrast to $\sim 34 \%$ and $\sim 37 \%$ nuclear signal reduction in xpo1-1 and mex67-5, respectively, an increased reduction ( $\sim 58 \%)$ is found in the xpo1-1 mex67-5 double mutant upon the temperature shift to $37^{\circ} \mathrm{C}$. This suggests an increased mislocalisation of the telomerase component in this double mutant. 

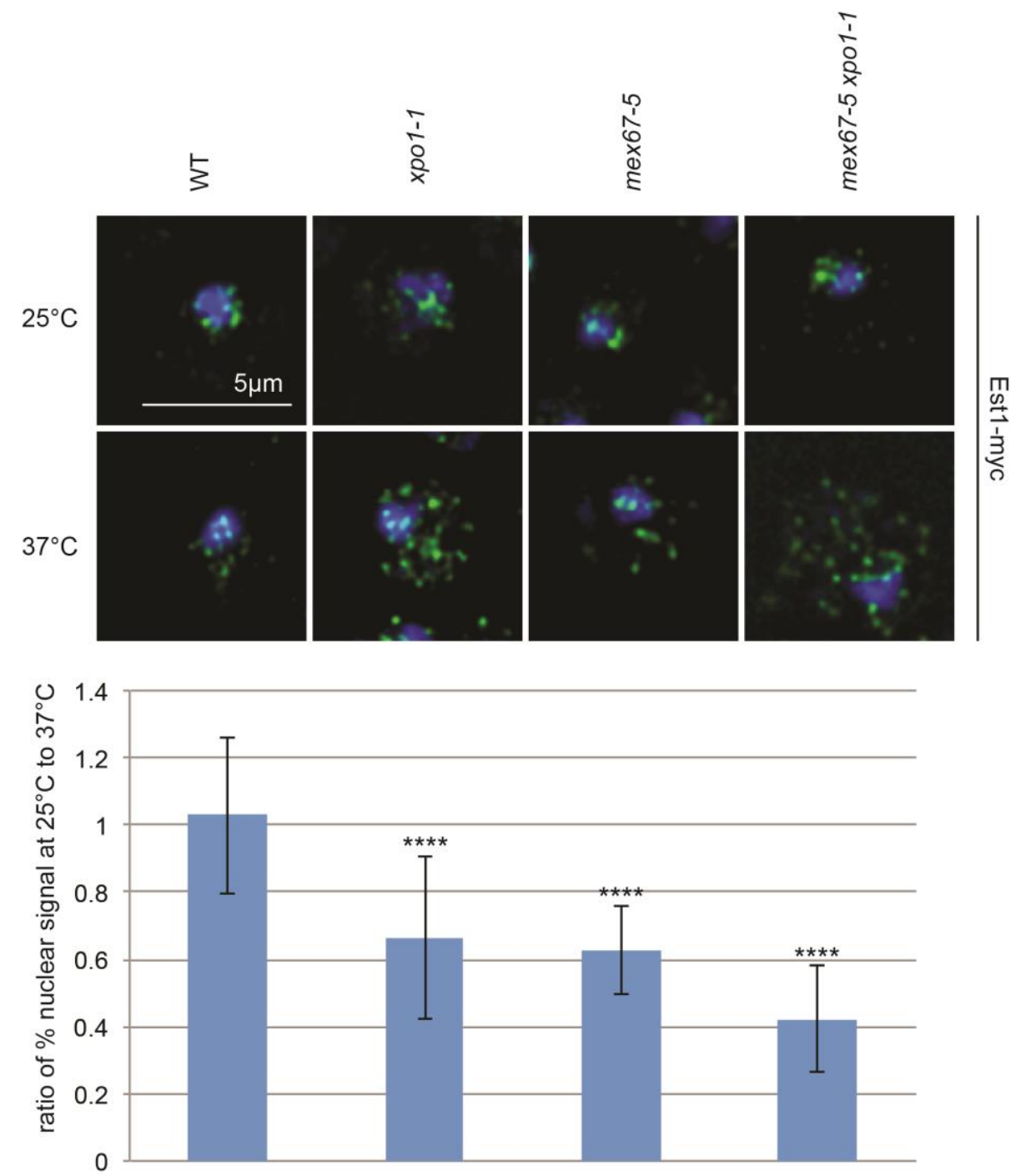

Figure 4.1.14 The xpo1-1 mex67-5 double mutant shows stronger cytoplasmic mislocalisation of the telomerase component than the single mutants. All indicated strain were grown to the logarithmic phase and were either retained at $25^{\circ} \mathrm{C}$ or shifted to $37^{\circ} \mathrm{C}$ for 1 hour. With immunofluorescence experiments (top panel), Est1-myc was detected by using mouse anti-myc antibodies and AlexFluor488 anti-mouse antibodies (green). The nuclei were stained with hoechst33342 (blue). The cells were imaged through 6-10 stacks $(0.2 \mu \mathrm{m} / \mathrm{stack})$ followed by deconvolution. To calculate the ratio of the nuclear signal of Est1, the nuclear Est1 signal was compared to that of the total Est1 signals. These ratios at $37^{\circ} \mathrm{C}$ was further compared to those at $25^{\circ} \mathrm{C}$ in a given strains and were showed in the diagram (bottom panel). For each condition at least 20 cells were calculated. At least three independent experiments were done, one of which is shown in the upper panel. Error bars indicate the standard deviation. P-value was calculated according to two-tailed, unpaired t-test (****: $p \leq 0.0001)$. 


\subsubsection{Telomere maintenance is impaired in the double mutant xpo1-1 mex67-5}

All of the findings shown above indicate that the xpo1-1 mex67-5 double mutant shows a stronger TLC1 nuclear export defect and an increased mislocalisation of the telomerase component. On the other side, these results additionally suggest a function of the mRNA export factor on TLC1 nuclear export. These results also support a coordination between the mRNA export pathway and the Crm1/Xpo1 pathway on TLC1 nuclear export. Although all these results support that TLC1 nuclear export utilises both the mRNA export and the $\mathrm{Xpo1/Crm1} \mathrm{pathways,} \mathrm{there} \mathrm{is} \mathrm{no} \mathrm{telomere} \mathrm{shortening} \mathrm{found} \mathrm{in} \mathrm{any} \mathrm{of} \mathrm{the} \mathrm{mutants} \mathrm{of} \mathrm{these}$ export factors alone. Since the xpo1-1 mex67-5 double mutant shows a very strong TLC1 export defect, it would be expected that telomere maintenance would also be impaired in this mutant. To test this hypothesis, southern blot experiments were performed to detect telomere length in this mutant (figure 4.1.15).

The strains wild type (HKY381), yku704 (HKY1073), xpo1-1 (HKY206), mex67-5 (HKY644) and xpo1-1 mex67-5 (HKY1353) were used for the southern blot experiments. Importantly, the temperature sensitive mutants expose their phenotypes only under non-permissive conditions, which are lethal. Therefore, the semi-permissive temperature of $32^{\circ} \mathrm{C}$, which was the highest temperature that allowed growth of the xpo1-1, mex67-5 double mutants, was used for cultivating the strains. Cells were initially grown at $25^{\circ} \mathrm{C}$ to the concentration of $1 \times 10^{8}$ cells $/ \mathrm{ml}$ and were regarded as the 0th generations. The cells were diluted 1:1000 into fresh media and grown again to the concentration of $1 \times 10^{8}$ cells $/ \mathrm{ml}$ at $32^{\circ} \mathrm{C}$. These cells were regarded as the cells that were grown for 10 generations. The cells were grown at $32^{\circ} \mathrm{C}$ for 0 , 30, 60 generations. The genomic DNA was extracted from the cells prior to an Xhol digestion. The digested genomic DNA was separated on 1.2\% agarose gels and transferred onto nylon membranes. The telomeric DNA was hybridised to a specific DIG labelled telomeric probe (HK1539) and detected by using DIG-High Prime DNA Labeling and Detection Starter Kit II from Roche.

Furthermore, to exclude the possibility that the telomere shortening phenotype in these 
A

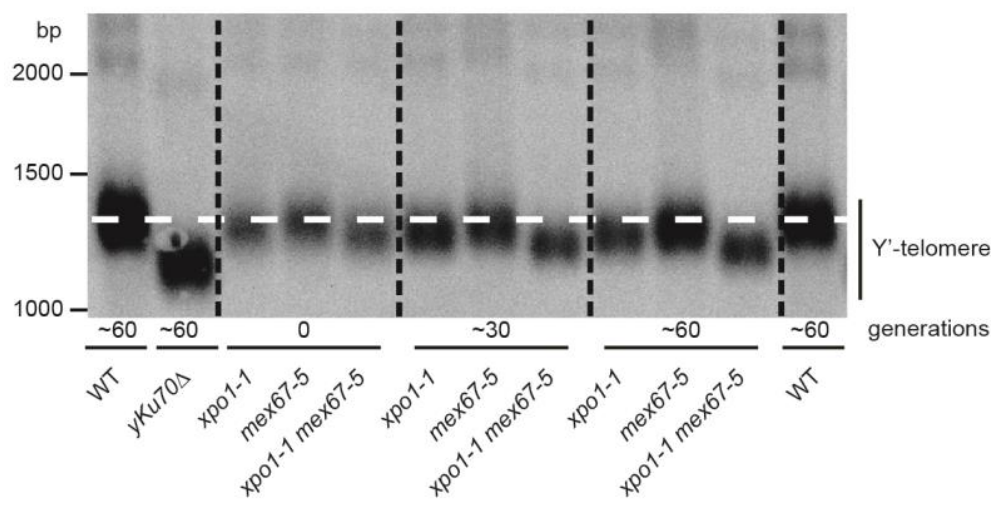

B

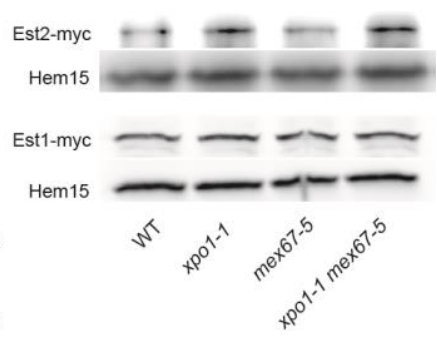

Figure 4.1.15 The xpo1-1 mex67-5 double mutant shows an impaired telomere maintenance at $32^{\circ} \mathrm{C}$. (A) The indicated strains were used for the southern blot experiments. Cells that were grown to the concentration of $1 \times 10^{8}$ cells $/ \mathrm{ml}$ were diluted 1:1000 into fresh media and grown again to the concentration of $1 \times 10^{8}$ cells $/ \mathrm{ml}$ at $32^{\circ} \mathrm{C}$. These cells were regarded as the cells that were grown for 10 generations. The cells were grown at $32^{\circ} \mathrm{C}$ for $0,30,60$ generations. The genomic DNA was extracted and Xhol digested. With southern blot experiments, the DNA that was hybridised to the DIG labeled telomeric oligo probes (HK1539) was detected by anti-DIG-AP antibodies and CSPD (Roche). The figure shows telomeres containing $Y^{\prime}$-subtelomeres. (B) The indicated strains were grown to the logarithmic phase at $32^{\circ} \mathrm{C}$. The protein levels of Est1 and Est2 were detected by using mouse anti-myc antibodies and anti-mouse-Hrp antibodies. Hem15 was used as loading control and was detected by using anti-Hem15 antibodies. At least three independent experiments were done, one of which is shown here.

mutants might be caused by expression defects of the telomerase components, western analyses was carried out to examine the protein levels of the telomerase components. For western analyses, the strains EST1-3xMYC (wild type, HKY1463), EST1-3xMYC xpo1-1 (HKY1464), EST1-3xMYC mex67-5 (HKY1465) and EST1-3xMYC xpo1-1 mex67-5 (HKY1466) were used for examining the protein levels of Est1. The strains EST2-3xMYC (wild type, HKY1467), EST2-3xMYC xpo1-1 (HKY1468), EST2-3xMYC mex67-5 (HKY1469) and EST2-3xMYC xpo1-1 mex67-5 (HKY1470) were used to examine the protein levels of Est2. All strains were grown to the logarithmic phase at $32^{\circ} \mathrm{C}$. The protein levels of Est1 and Est2 were detected by using mouse anti-myc antibodies and anti-mouse-HRP antibodies. Hem15 that was also used in section 4.2.1.3 served as the reference and detect by rabbit anti-Hem15 antibodies and anti-rabbit-HRP antibodies.

Strikingly, the southern blot experiments indicate that the xpo1-1, mex67-5 double mutant shows a clear telomere shortening after growing for about 30 generations at $32^{\circ} \mathrm{C}$ and this 
shortening is even enhanced after around 60 generations (figure 4.1.15A). In contrast to that, the mex67-5 mutant shows no telomere shortening and the xpo1-1 mutant shows very mild telomere shortening. Besides, the western blot experiments show that Est1 and Est2 are not degraded at $32^{\circ} \mathrm{C}$ in all strains (figure $4.1 .15 \mathrm{~B}$ ). In addition, a stable $T L C 1$ transcription level at $32^{\circ} \mathrm{C}$ was also identified (Daniel Becker and Heike Krebber). These results indicate that the TLC1 export defects lead to defective telomere maintenance. From these experiments, genetic evidence is also obtained, supporting that the mRNA export pathway cooperates with $\mathrm{Crm} 1 / \mathrm{Xpo1}$ to mediate the nuclear export of $T L C 1$. 


\subsubsection{Xpo1/Crm1 is directly involved in TLC1 nuclear export}

Since xpo1-1 indirectly leads to mRNA nuclear export defect (AskjaerStade et al., 1997), whether xpo1-1 leading to TLC1 nuclear accumulation is direct remains unclear. To answer this question, fluorescent in situ hybridisation and southern blot experiments were performed (figure 4.1.16).

Overexpression of DBP5/RAT8 is able to rescue the mRNA export defect of xpo1-1 (Hodge et al., 1999). To identify the role of Xpo1/Crm1 on transporting $T L C 1$, an overexpression plasmid of DBP5/RAT8 was used to rescue the mRNA nuclear export in xpo1-1. This plasmid contained the DBP5/RAT8 gene under control of a galactose promoter (pGAL1-RAT8-MYC, $2 \mu$, URA3, pHK649) and was used for transforming the strains wild type (HKY381), yku70 4 (HKY1073), xpo1-1 yku704 (HKY1444), mex67-5 yku704 (HKY1397) and xpo1-1 mex67-5 yku704 (HKY1445). Fluorescent in situ hybridisation experiments were performed in the transformed strains (figure 4.1.16A). All strains were initially inoculated in raffinose containing media. Galactose or glucose is used as carbon source and for inducing or repressing the overexpression of DBP5/RAT8 from the plasmid, respectively. The induced or repressed strains were grown overnight to the logarithmic phase and shifted to $37^{\circ} \mathrm{C}$ for 1 hour. Poly $(A)^{+}$RNA or TLC1 molecules were detected by using a Cy3-oligod $(T)_{50}$ probe (Biospring) or Cy3 labelled specific TLC1 oligo probes (HK1761, HK1789, HK1790).

In addition, southern blot experiments were carried out to identify the length of the telomeres in the double mutant xpo1-1 mex67-5 in the presence of overexpressed DBP5/RAT8 (figure 4.1.16B). All strains wild type (HKY381), yku704 (HKY1073), xpo1-1 (HKY206), mex67-5 (HKY644) and xpo1-1 mex67-5 (HKY1353) were transformed with the plasmid pHK649 (pGAL1-RAT8-MYC, $2 \mu$, URA3). Transformed strains were grown at $32{ }^{\circ} \mathrm{C}$ with addition of either glucose or galactose for about 60 generations. The cells were harvested and the southern blot experiments were performed as mentioned in section

\subsubsection{3.}

The fluorescent in situ experiments show that although defect of poly $(A)^{+}$RNA export is rescued in xpo1-1 by overexpression of DBP5/RAT8, the TLC1 nuclear mislocalisation still 
remains, indicating that the defect of $T L C 1$ translocation in xpo1-1 is due to its impaired $\mathrm{Crm1/Xpo1} \mathrm{function} \mathrm{rather} \mathrm{than} \mathrm{the} \mathrm{improper} \mathrm{regulation} \mathrm{of} \mathrm{mRNA} \mathrm{export.} \mathrm{The} \mathrm{southern} \mathrm{blot}$ experiments show that suppression of the xpo1-1 phenotype does not rescue the defect in telomere maintenance caused by the xpo1-1, mex67-5 double mutants. These findings further provide evidence that unlike mRNA nuclear export, $\mathrm{Crm} 1 / \mathrm{Xpo1}$ is directly involved in TLC1 nuclear export.

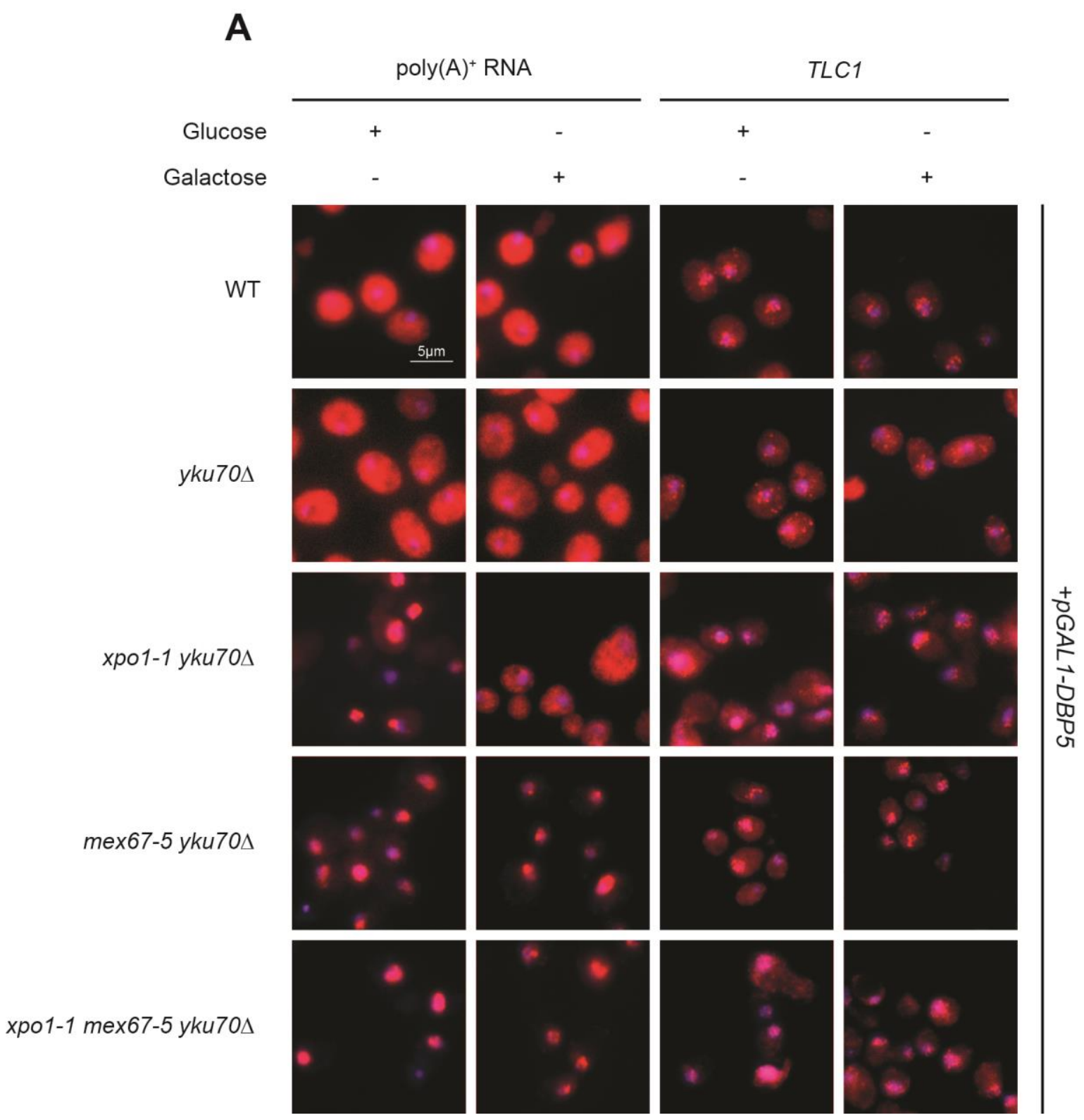

Figure legend see next page 
B

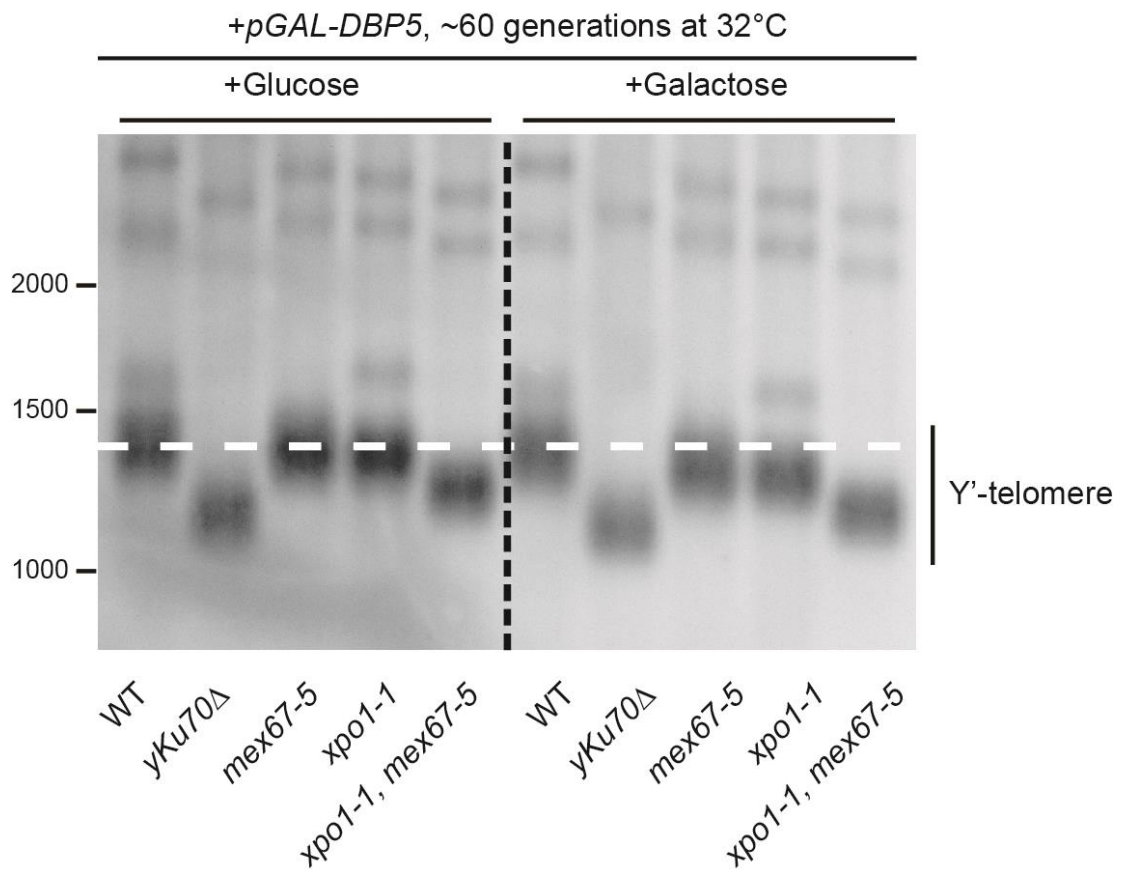

Figure 4.1.16 Overexpression of DBP5/RAT8 does not rescue the TLC1 nuclear accumulation in xpo1-1 and the telomere shortening in the xpo1-1 mex67-5 double mutants. (A) All indicated strains were transformed with the plasmid pHK649 (pGAL1-RAT8-MYC, $2 \mu, U R A 3)$. Fluorescent in situ hybridisation experiments were performed in the transformed strains. All strains were initially inoculated in raffinose containing media. Overexpression of DBP5/RAT8 was either induced or repressed in all strains overnight by adding galactose or glucose, respectively. The strains were grown to the logarithmic phase and shifted to $37^{\circ} \mathrm{C}$ for 1 hour. Poly $(A)^{+}$RNA or TLC1 molecules (red) were detected by using Cy3-oligod $(\mathrm{T})_{50}$ probe (Biospring) or Cy3 labelled specific TLC1 oligo probes (HK1761, HK1789, HK1790). The nuclei were stained with hoechst33342 (blue). (B) The cells were grown at $32{ }^{\circ} \mathrm{C}$ in the presence of either glucose or galactose for about 60 generations. The genomic DNA was extracted and Xhol digested. With southern blot the DNA that was hybridised to the DIG labeled telomeric oligo probes (HK1539) was detected by anti-DIG-AP antibodies and CSPD (Roche). Figure shows telomeres containing $\mathrm{Y}^{\prime}$-subtelomeres. At least three independent experiments were done, one of which is shown here. 


\subsection{The processing of TLC1}

\subsubsection{The transcription and processing defects of TLC1 in the mtr10 $\triangle$ strain is not due to its effect on TLC1 transport}

Mtr10 is a nuclear import receptor that mediates $T L C 1$ nuclear import (Ferrezuelo et al., 2002). Previous investigation showed that the transcription level of $T L C 1$ was greatly reduced in mtr10 (Ferrezuelo et al., 2002), suggesting a feedback effect of the nuclear import of TLC1 might regulate its transcription. To test this hypothesis, qRT-PCR experiments were performed in the TLC1 mislocalisation mutants (figure 4.2.1).

In addition to the mtr10 $\Delta$ strain, other knockout strains that showed cytoplasmic mislocalisation of TLC1 (Ferrezuelo et al., 2002; Gallardo et al., 2008) were also used in the experiments. All strains wild type (HKY381), est2 $\Delta$ (HKY1072), yku70 $\Delta$ (HKY1073), tel1 $\Delta$ (HKY1075), mre114 (HKY1077), mtr104 (HKY82) and pdr64 (HKY209) were grown to the

A

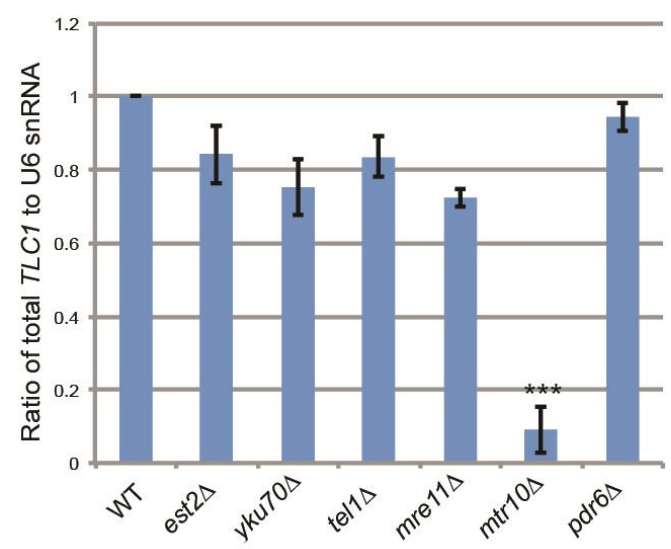

B

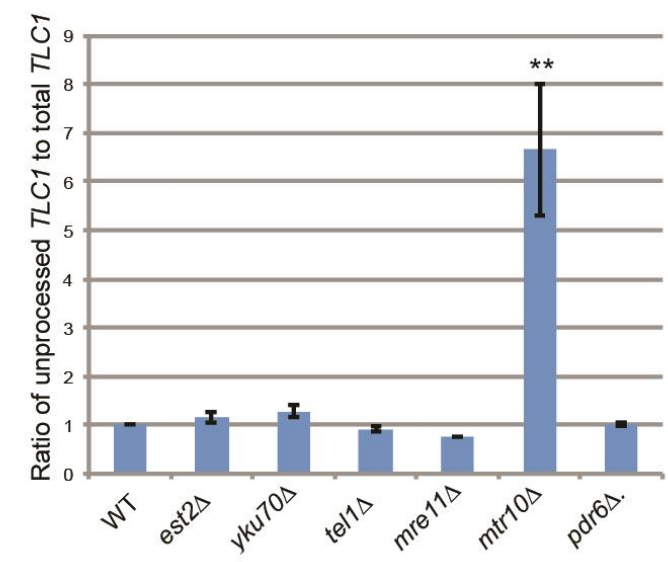

Figure 4.2.1 Quantification of TLC1 transcription and processing in the mutants that show a cytoplasmic mislocalisation of $\boldsymbol{T L C 1}$. All indicated strains were grown to the logarithmic phase and the total RNAs were extracted. The amounts of the total TLC1, the unprocessed TLC1 and the U6 SnRNA were measured via qRT-PCR by using specific primer pairs HK1385+HK1386, HK1382+HK1384 and HK1404+HK1405 respectively. (A) To evaluate the TLC1 transcription level, the amount of the total TLC1 was compared to that of the U6 snRNA and the ratios were set into relation with that of the wild type. (B) To evaluate $T L C 1$ processing rates, the amount of the unprocessed $T L C 1$ was compared to that of the total TLC1 and the ratios were set into relation with that of the wild type. At least three independent experiments were done. Error bars indicate the standard deviation. P-value was calculated according to two-tailed, paired t-test $\left({ }^{* *}: 0.001 \leq p \leq 0.01 ; * * *: 0.0001 \leq p \leq 0.001\right)$. 
logarithmic phase and the total RNA was extracted from the cells. The amount of the total TLC1 and unprocessed TLC1 was quantified by using qRT-PCR with the primer pair HK1385+HK1386 and HK1382+HK1384, respectively. The U6 snRNA was quantified as a transcription control by using the primer pair HK1404+HK1405 as mentioned in section 4.1.1.4. To evaluate the transcription levels of $T L C 1$, the amount of the total $T L C 1$ were compared to that of the snRNA U6 and the ratios were set into relation to that of the wild type. To evaluate the processing of $T L C 1$, the amount of unprocessed TLC1 was compared to that of the total TLC1 and the ratios were set into relation to that of the wild type.

Unexpectedly, the experiments show that although all these factors are involved in the TLC1 nuclear import, Mtr10 is the only factor that affects the transcription of TLC1. Besides, the mtr10 $\triangle$ strain possesses not only a TLC1 transcription level reduction, but also an impaired TLC1 processing. These defects are not found in the other mutants that affect TLC1 localisation. Therefore, this result suggests a unique function of Mtr10 in TLC1 transcription and processing within these factors. Since Mtr10 is known as a nuclear import receptor (Senger et al., 1998), it can be proposed that unlike the other factors, Mtr10 might import not only the telomerase complex, but also factors necessary for TLC1 transcription and processing, into the nucleus. 


\subsubsection{The factors involved in TLC1 processing are identified by qRT-PCR analyses}

The results from section 4.1.1.4 and 4.1.3.1 suggest that TLC1 maturation might be completed in the nucleus. Therefore, it can also be concluded that TLC1 maturation is mediated by the nuclear RNA processing factors. To identify which factors mediate TLC1 processing, qRT-PCR analyses were performed (figure 4.2.2).

The experiments were performed by using the mutants of the nuclear RNA processing factors, e.g. components of the ribonuclease, components of the TRAMP complex and the splicing factors, etc. After all strains were grown to the logarithmic phase, wild type (HKY381)

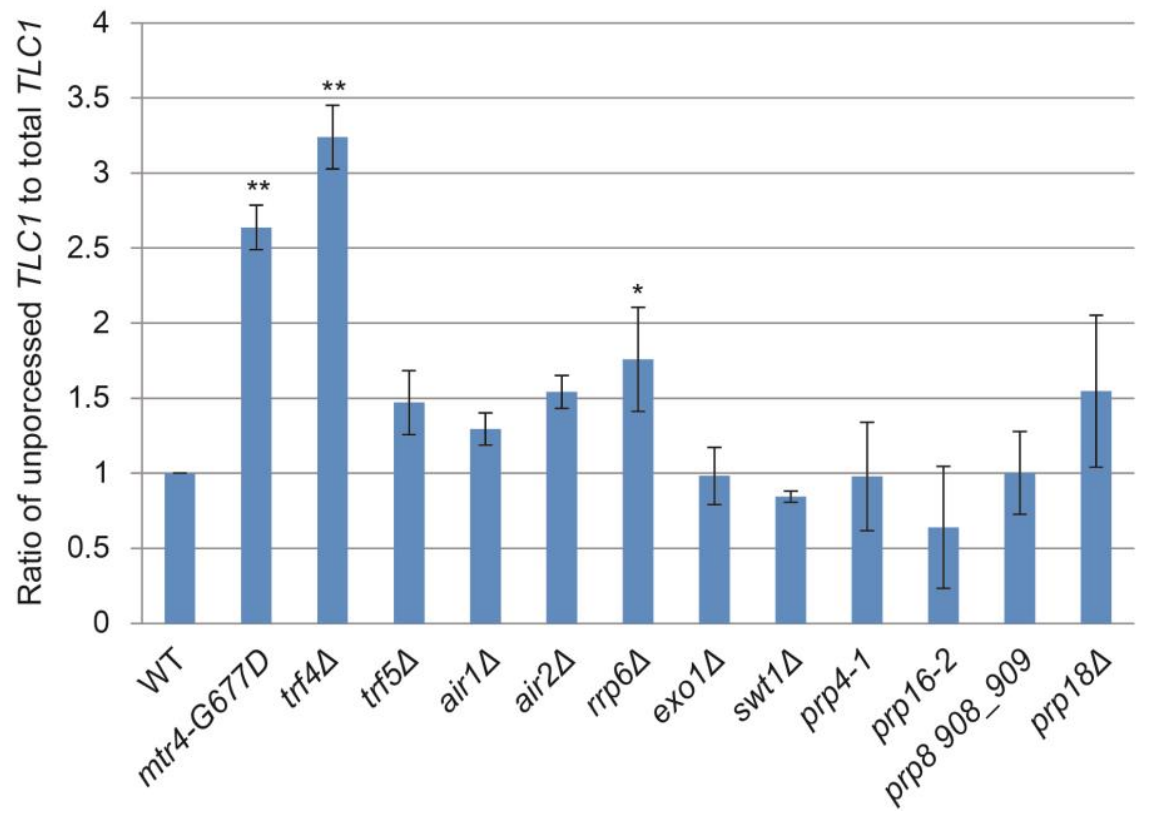

Figure 4.2.2 Potential TLC1 processing factors are identified by using qRT-PCR experiments. All indicated strains were grown to the logarithmic phase. Wild type (HKY381), mtr4-G677D (HKY428), prp4-1 (HKY305), prp16-2 (HKY306), prp8 908_909 (HKY1008) were shift to $37^{\circ} \mathrm{C}$ for 1 hour and rrp64

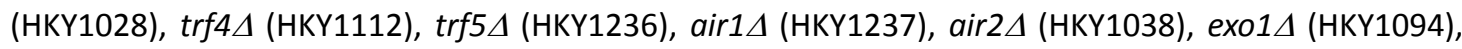
swt1 $\triangle$ (HKY1111) were incubated at $25^{\circ} \mathrm{C}$. The total RNAs were extracted from the cells. The qRT-PCR analyses were performed to measure the amount of total TLC1 and unprocessed TLC1 with the primer pairs HK1385+HK1386 and HK1382+HK1384, respectively. To evaluate TLC1 processing, the amounts of unprocessed $T L C 1$ were compared to those of the total $T L C 1$ and the ratios were set into relation to the wild type. At least three independent experiments were done. Error bars indicate the standard deviation. P-value was calculated according to two-tailed, paired t-test $(*$ : $0.01 \leq \mathrm{p} \leq 0.05$; **: $0.001 \leq p \leq 0.01)$. 
and the temperature sensitive mutants mtr4-G677D (HKY428), prp4-1 (HKY305), prp16-2 (HKY306) and prp8 908_909 (HKY1008) were shifted to $37^{\circ} \mathrm{C}$ for 1 hour. The knockout strains rrp64 (HKY1028), trf4A (HKY1112), trf5 (HKY1236), air14 (HKY1237), air24 (HKY1038),

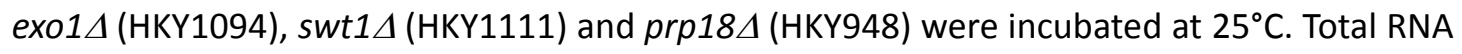
was extracted from these strains and further used for the qRT-PCR analyses. In qRT-PCR, the primer pair HK1385+HK1386 was used to measure the total TLC1 and the primer pair HK1382+HK1384 was used to measure the unprocessed TLC1. To evaluate the processing of $T L C 1$, the amount of the unprocessed TLC1 was compared to that of the total TLC1 and the ratios were set into relation to that of the wild type.

The results indicate that unlike telomerase RNA in Schizosaccharomyces pombe (Box et al., 2008) and Trypanosoma brucei (Sandhu et al., 2013), TLC1 does not undergo splicing in S. cerevisiae. In addition, Swt1, an RNA endonuclease involved in RNA turnover in the nucleus (Skruzny et al., 2009), and Exo1, an exonuclease mainly responsible for DNA recombination (Fiorentini et al., 1997; Tran et al., 2002), show no influence on the processing of TLC1. In contrast, clear defects of the processing of TLC1 are shown in the TRAMP component mutants (mtr4-G677D, trf4A) as well as the nuclear exosome component knockout strain (rrp64). Consistently, recent published data also show that Rrp6 and Lrp1 are involved in the processing of TLC1 (Coy et al., 2013).

The yeast nuclear exosome is composed of the exo-10 core complex, Rrp6 and Lrp1 (Synowsky et al., 2009). So far, no data are available to show if the catalytic core component of the exo-10 complex, Dis3 (Mitchell et al., 1997), is involved in the processing of TLC1. Besides, since Dis3 possesses both endo- and exo-nuclease activities (Mitchell et al., 1997), it is also unclear which activity of Dis3 could be responsible for the processing of TLC1. To answer these questions, qRT-PCR experiments were performed (figure 4.2.3).

Using the strain (GAL::dis3, HKY1290) in which the genomic DIS3 promoter was replaced by a GAL promoter, expression of DIS3 is able to be repressed by glucose (Schneider et al., 2009). The strain wild type (HKY381) and GAL::dis3 (HKY1290) were initially inoculated in galactose containing media. The GAL::dis3 culture was divided into two equal portions. One portion of the GAL::dis3 culture and the wild type culture were kept in galactose containing media and 
A
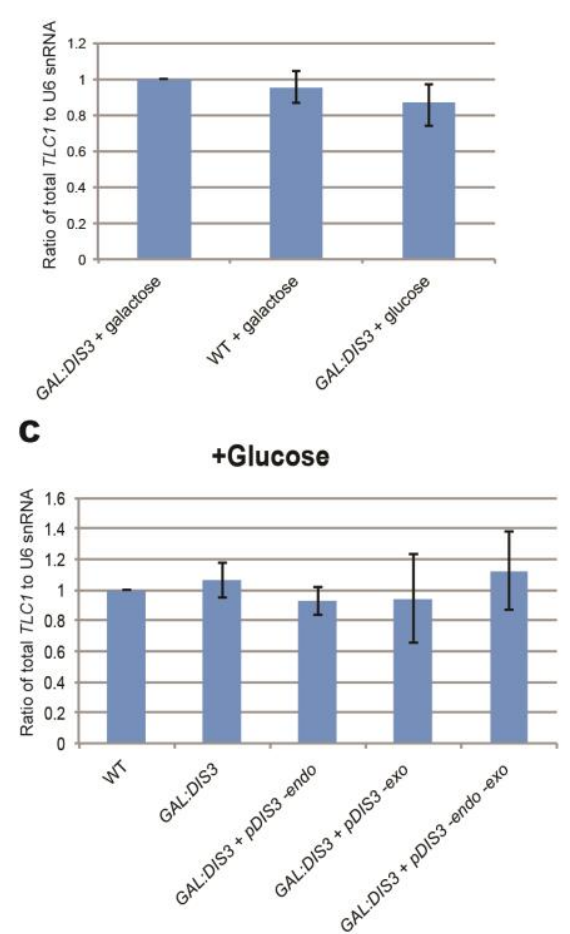

B

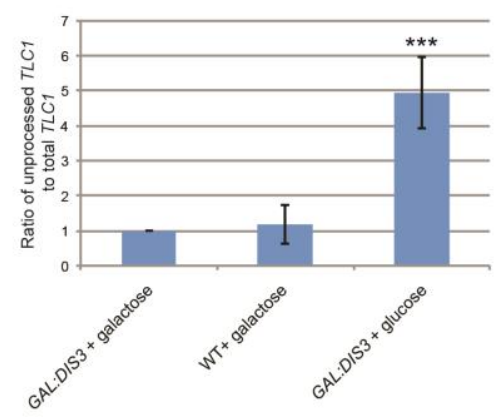

D

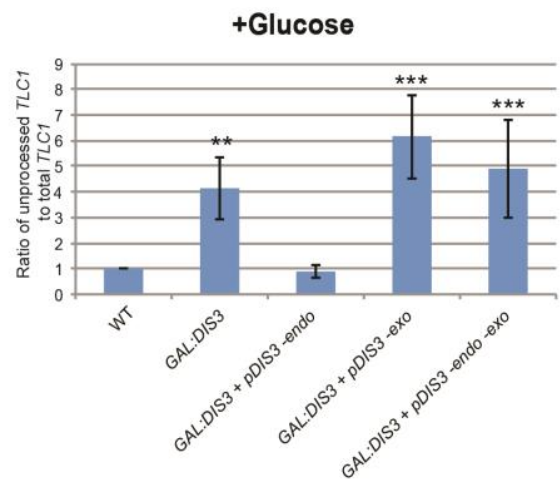

Figure 4.2.3 Dis3 is involved in the processing of TLC1 and this activity might be mainly from its exonuclease activity. (A, B) The indicated strains were grown in galactose containing media. Half of the GAL::dis3 cells and the wild type cells were kept in galactose containing media and grown for 24 hours to the logarithmic phase. The other half of the GAL::dis3 cells were grown in glucose containing media for 24 hours to the logarithmic phase. Total TLC1 and unprocessed TLC1 were quantified by qRT-PCR with the primer pairs HK1385+HK1386 and HK1382+HK1384, respectively. The U6 snRNA was used as the reference RNA and quantified with the primer pair HK1404+HK1405. TLC1 transcription levels were calculated through the ratios of the total TLC1 to the U6 snRNAs (A). The processing of TLC1 was evaluated through the ratios of the unprocessed TLC1 to the total TLC1 (B). (C, D) Wild type strain (HKY381) bearing empty vector ( $p R S 315-L E U 2$, pHK87) and the strain GAL::dis3 (HKY1290) bearing empty vector (pHK87), or one of the three plasmids ( $p$ DIS3-exo, pHK1353; pDIS3-endo, pHK1354; pDIS3-exo-endo, pHK1355) were cultivated in galactose containing media. Subsequently expression of wild-typic DIS3 gene in GAL::dis3 strains is repressed by adding glucose for 24 hours. TLC1 transcription (C) and processing (D) were evaluated as mentioned above. At least three independent experiments were done. Error bars indicate the standard deviation. P-value was calculated according to two-tailed, paired t-test $(* *: 0.001 \leq p \leq 0.01$; $* * *$ : $0.0001 \leq \mathrm{p} \leq 0.001)$.

grown for 24 hours to the logarithmic phase. Glucose was added to the other portion of the GAL::dis3 culture and it was also grown for 24 hours to the logarithmic phase. Total RNA was extracted from the cells. Total TLC1 and unprocessed TLC1 were quantified by qRT-PCR with the primer pairs HK1385+HK1386 and HK1382+HK1384, respectively. The U6 snRNA was used as the reference RNA and quantified with the primer pair HK1404+HK1405. TLC1 
transcription and processing were evaluated (figure 4.2.3 A,B) as mentioned in section

\subsubsection{4.}

Furthermore, three plasmids were used to identify the functional domain of Dis3 for the processing of TLC1. All three plasmids contain mutated DIS3 genes. One plasmid ( $p D I S 3-e n d o$, LEU2, pHK1354) produces defective Dis3 proteins without its endo-nuclease activity; one plasmid ( $p$ DIS3-exo, LEU2, pHK1353) produces defective Dis3 proteins without its exo-nuclease activity; and the third plasmid (pDIS3-exo-endo, LEU2, pHK1355) produces defective Dis3 proteins without both endo- and exo-nuclease activities (Schneider et al., 2009). The wild type strain (HKY381) was transformed with the empty vector (pRS315-LEU2, CEN, pHK87) and the strain GAL::dis3 (HKY1290) was transformed with the empty vector (pHK87), or one of the three plasmids (pHK1353, pHK1354, pHK1355). All these transformed strains were initially inoculated in galactose containing media. Subsequently glucose was added to the cultures to repress the expression of the wild-typic DIS3 gene in the GAL::dis3 strains. The strains were cultivated for 24 hours in this media and grown to the logarithmic phase. Total RNA was extracted from the cells. TLC1 transcription and processing were evaluated (figure 4.2.3 C,D).

The qRT-PCR analyses show that the processing of TLC1 is defective upon repression of DIS3 expression, suggesting a role of Dis3 in the processing of $T L C 1$ (figure 4.2.3A, B). Furthermore, the results also indicate that only in the DIS3-exo mutants, the processing of TLC1 is impaired, suggesting the involvement of the exonuclease activity of Dis3 in this procedure (figure 4.2.3C, D). In addition, the DIS3-exo mutant shows a similar level of impairment of the processing of TLC1 as shown in the DIS3-endo-exo strain and the DIS3 expression repressed strain (GAL::dis3 in glucose containing media), further pointing out that the catalytic function of Dis3 on the processing of TLC1 is specific from its exo-nuclease activity rather than the endo-nuclease activity. 


\subsubsection{The factors involved in the processing of TLC1 are mis-localised in the mtr10 $\Delta$ mutant}

Previously published data (Coy et al., 2013) show that the nuclear exosome and the TRAMP complex might be involved in the processing of TLC1. This opinion is also supported by our results in section 4.2.2. Furthermore, the results from section 4.2.1 suggest that Mtr10 might transport factors that are necessary for the processing of TLC1. Therefore, immunofluorescence experiments were performed to identify the localisation of the nuclear exosome components and the TRAMP complex components in $m$ tr10 $\Delta$ (figure 4.2.13).

In the immunofluorescence experiments, wild type (HKY381) and mtr10 4 (HKY82) cells were

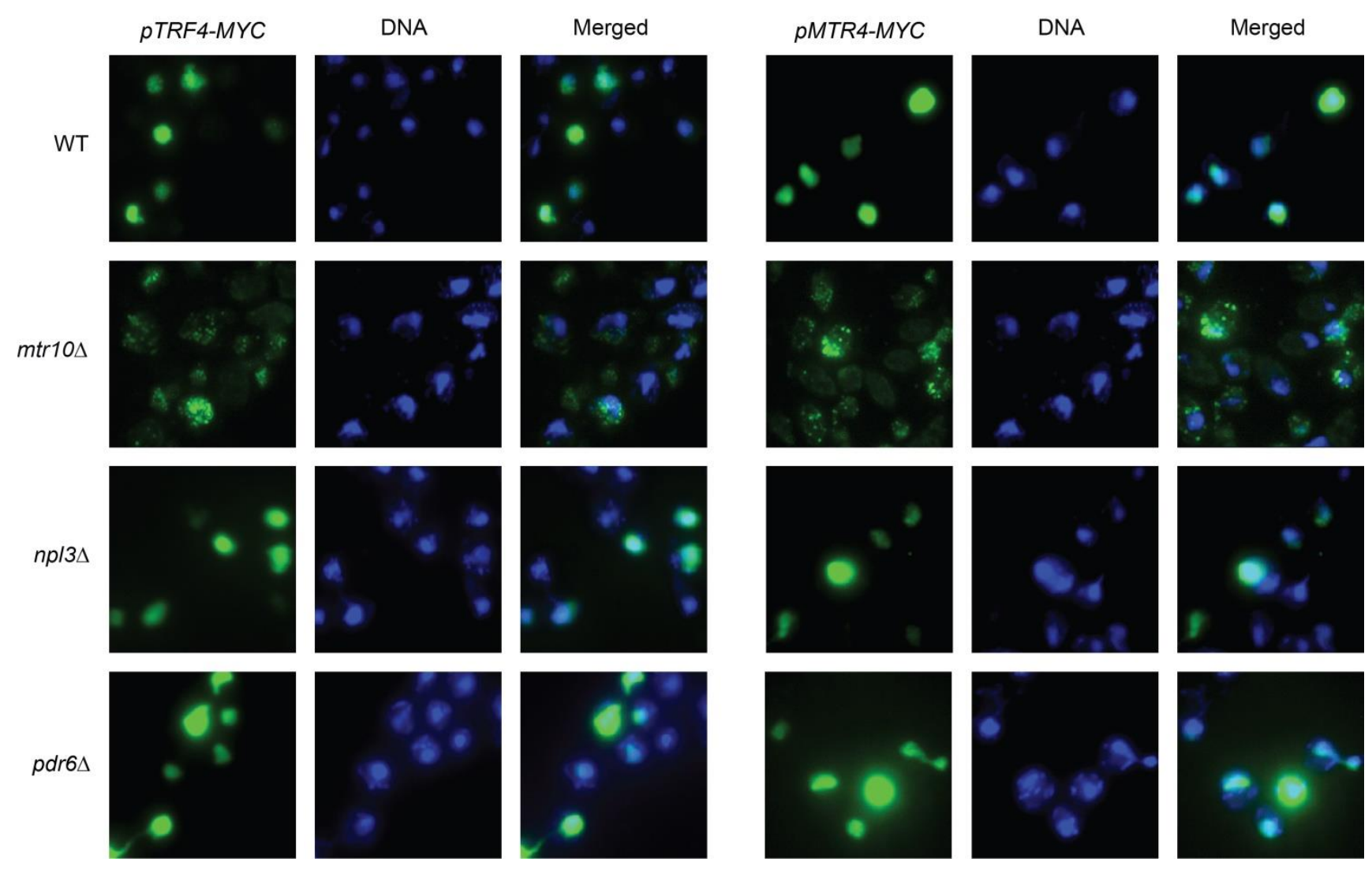

Figure 4.2.4 Trf4 and Mtr4 are mis-localised in mtr10 $\Delta$. The strains wild type (HKY381), mtr10 $\Delta$ (HKY82), np/3A (HKY380) and pdr64 (HKY309) bearing the plasmid pTRF4-MYC (pHK1238) or pMTR4-MYC (pHK1240) were grown to the logarithmic phase. Myc tagged Trf4 or Mtr4 was detected by mouse anti-myc antibodies and AlexaFluor488 anti-mouse antibodies (green). The nuclei were stained with hoechst33342 (blue). Merged figures are used for co-localisation study. At least three independent experiments were done, one of which is shown here. 
used. Besides, since Pdr6 is involved in TLC1 nuclear import and Npl3 is one of the Mtr10 nuclear import targets, pdr6 4 (HKY309) and np/3A (HKY380) were also used in the experiments. The strains were transformed with pTRF4-MYC (pHK1238) or pMTR4-MYC (pHK1340) plasmids. Transformed strains were grown to the logarithmic phase. Myc tagged proteins were detected with mouse anti-myc antibodies and AlexaFluor488 sheep anti-mouse secondary antibodies. Nuclei were stained with hoechst33342.

The results show that in the wild type, Trf4 and Mtr4 are located to the nucleus, indicating that they are nuclear proteins. In contrast, in the mtr10 $\Delta$ strain these factors are mislocalised to the cytoplasm, suggesting that Mtr10 mediates their nuclear import. Furthermore, this mislocalisation is unique for $m \operatorname{tr} 10 \Delta$ since the other nuclear receptors, e.g. Pdr6, or the Mtr10 transport target, Npl3, do not cause this mislocalisation. In addition, mislocalisation of Dis3 in mtr10 $\Delta$ was also identified (Lea Steffen and Heike Krebber). These findings indicate a unique function for Mtr10 in transporting the nuclear exosome components and the TRAMP complex components. 


\subsubsection{Mtr10 physically interacts with factors that are involved in the processing of TLC1}

The studies of localisation of the TRAMP complex components and the nuclear exosome component show that these factors are mislocalised to the cytoplasm in $m t r 10 \Delta$. This finding suggests potential physical interactions between Mtr10 and these factors. To identify the interactions, co-immunoprecipitation experiments were performed (figure 4.2.5).

The wild type strain (HKY381) and the DIS3-GFP (HKY1172) strain were transformed with the plasmids pMTR10-TAP (pHK413) and pTRF4-MYC (pHK1238). Transformed strains were grown to the logarithmic phase. Mtr10-TAP was precipitated using IgG coupled sepharose

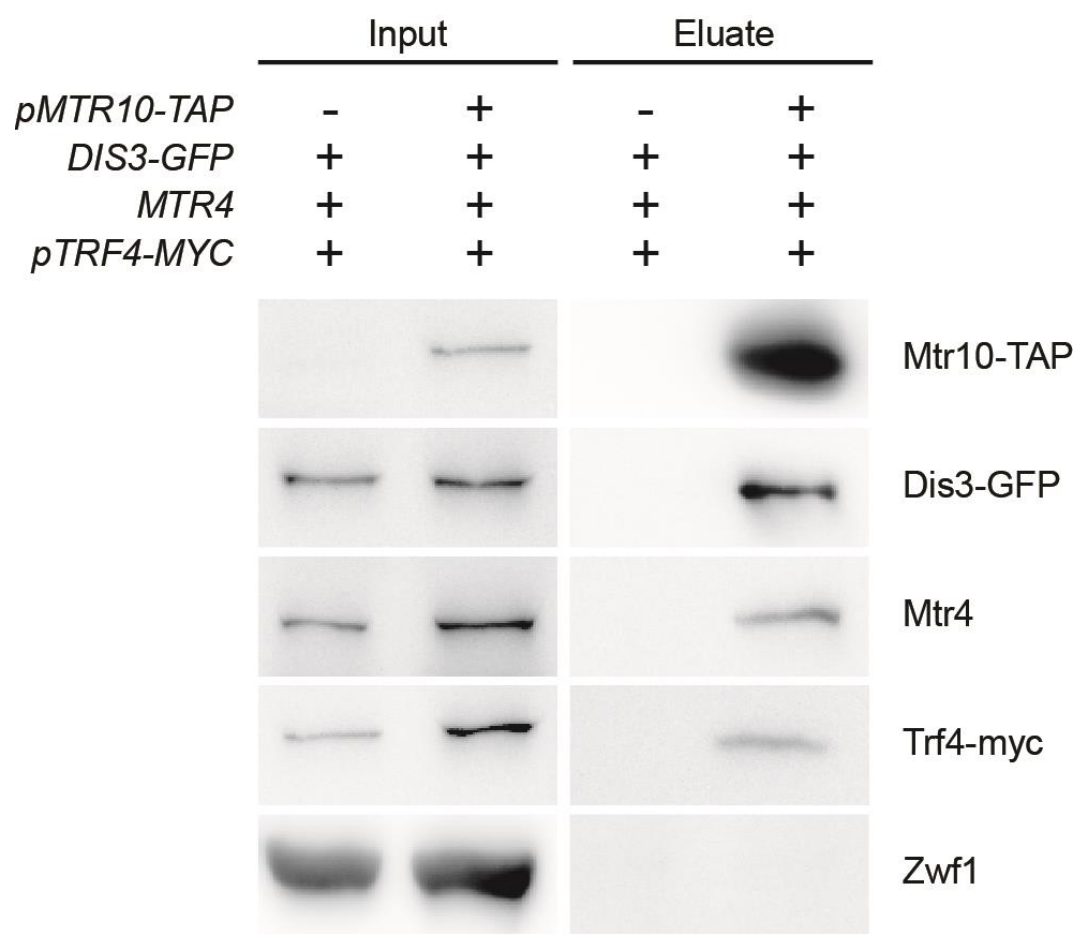

Figure 4.2.5 Mtr10 interacts with the TRAMP complex components and an exosome component. The strains wild type (HKY381) and DIS3:GFP (HKY1172) were transformed with the plasmids pMTR10-TAP (pHK413) and pTRF4-MYC (pHK1238). Mtr10-TAP was precipitated by using IgG coupled sepharose beads (GE Healthcare) in the presence of RNaseA $(0.1 \mathrm{mg} / \mathrm{ml})$. Its interaction partners were analysed by western blots. The whole cell lysates were used as input controls. Dis3-GFP was detected by anti-GFP antibodies. Trf4-myc was detected by anti-myc antibodies. Mtr4 was detected by anti-Mtr4 antibodies. Mtr10 is detected by any of these antibodies. Zwf1 was used as loading and washing control and was detected by anti-Zwf1 antibodies. At least three independent experiments were done, one of which is shown here. 
beads (GE Healthcare). RNaseA (final concentration: $0.1 \mathrm{mg} / \mathrm{ml}$ ) was used during the experiments to remove potential RNA mediated interactions. The eluates were analysed by western blots. Dis3 was detected by using anti-GFP antibodies and Trf4 was detected by using anti-myc antibodies and Mtr4 was detected by using anti-Mtr4 antibodies and Mtr10 is detected by any of these antibodies. Zwf1, a cytoplasmic protein used in section 4.1.1.2, was used as the loading control and detected by using anti-Zwf1 antibodies.

The results show physical interactions between Mtr10 and these exosome or TRAMP complex components. These findings support that both TRAMP complex components and an exosome component are transported via Mtr10. Moreover, these findings also indicate that the processing and transcription of $T L C 1$ in $m \operatorname{tr} 10 \Delta$ could be due to the transport defects of the TRAMP components and the exosome component. 


\subsection{Conclusions}

This thesis led to a better understanding of the life cycle of $T L C 1$.

Fluorescent in situ hybridisation experiments identified that $T L C 1$ accumulates in the nucleus of the mRNA export mutants, suggesting that not only known Xpo1/Crm1, but also the mRNA export factors are involved in TLC1 nuclear export. This finding is further supported by the cell fractionation experiments showing cytoplasmic TLC1 deficiency in the mRNA export mutants and by the co-immunoprecipitation experiments showing physical interactions between TLC1 and the mRNA export factors. Furthermore, the RNA co-immunoprecipitation and qRT-PCR analyses indicate that the processing of TLC1 is completed in the nucleus and the subsequent nuclear export is mediated by the cooperation of Xpo1/Crm1 and the mRNA export factors. In addition, immunofluorescence and immunoprecipitation experiments show that the TLC1 nuclear export defect impairs the formation of the telomerase complex and the localisation of the telomerase components. Moreover, the xpo1-1 mex67-5 double mutant shows a stronger TLC1 nuclear export defect, an increased TLC1 processing rate and an enhanced mislocalisation of the telomerase components than in the single mutants, supporting a model in which Xpo1/Crm1 and the mRNA export factors cooperate to transport TLC1. In support of these findings, in the xpo1-1 mex67-5 double mutant a telomere maintenance defect is observed confirming the genetic interaction between Xpo1/Crm1 and Mex67. Finally, the fluorescent in situ hybridisation and southern blot experiments show that Xpo1/Crm1 is directly involved in TLC1 nuclear export.

Furthermore, qRT-PCR experiments reveal the transcription and processing defects of TLC1 in mtr10 $\triangle$ and propose that in addition to TLC1 transport, Mtr10 might possess a unique function in the transcription and processing of TLC1. QRT-PCR experiments further show that mutations of the genes of the TRAMP complex components and the exosome component lead to defects in the processing of $T L C 1$, suggesting involvements of these factors in the processing of TLC1. Further immunofluorescence experiments show cytoplasmic mislocalisations of the TRAMP complex components and the exosome component in mtr10 logically connecting these proposals and suggesting a possibility of the transport of these 
factors via Mtr10. In support of this hypothesis, the physical interaction studies reveal the interactions between Mtr10 and the TRAMP complex components or the exosome component.

Taken together, our knowledge about the transport and maturation of TLC1 is extended and the model of the life cycle of TLC1 is demonstrated in figure 4.3.1.

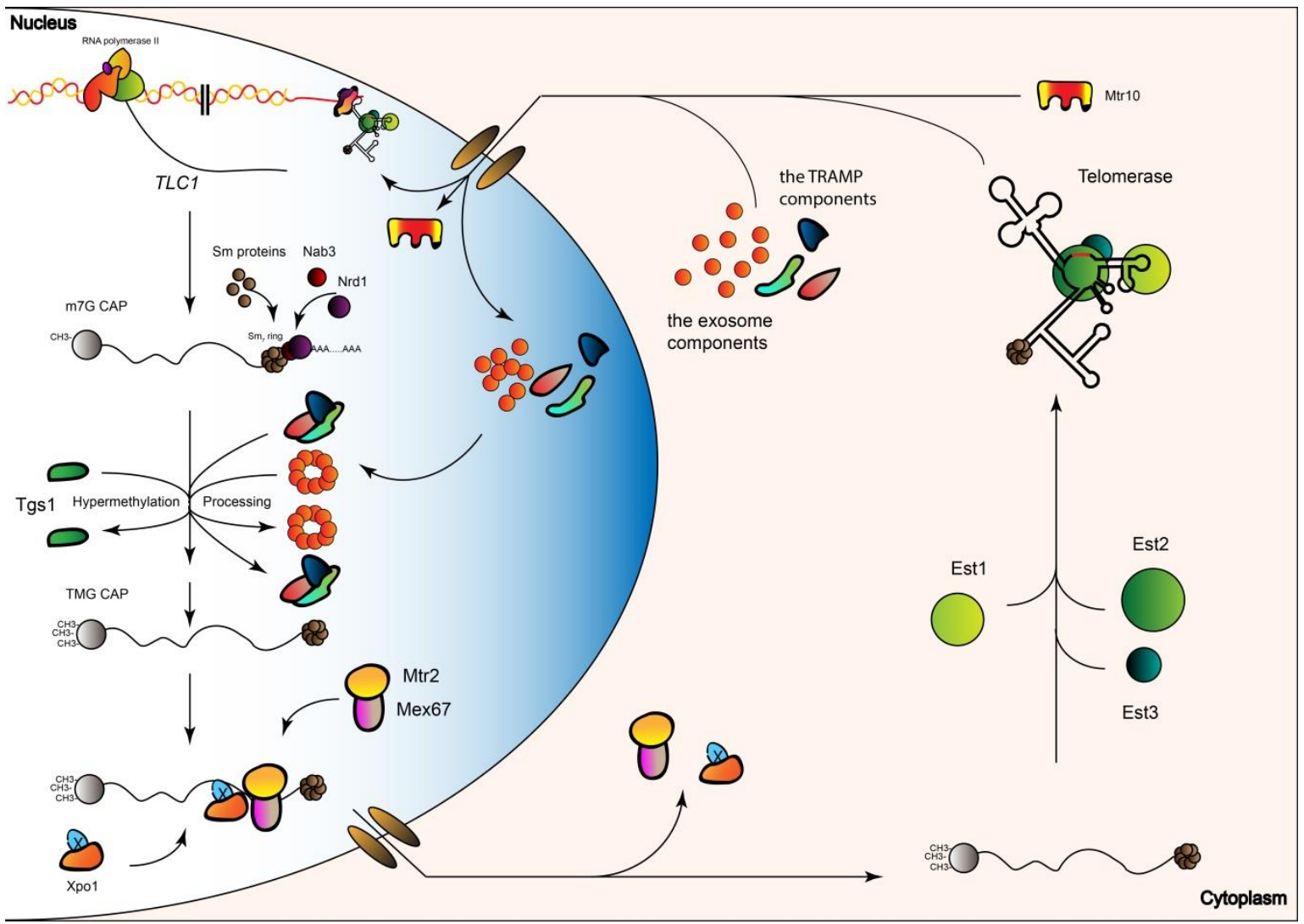

Figure 4.3.1 Model of the transport and maturation of TLC1. TLC1 is generated by RNA polymerase II with a $\mathrm{m}^{7} \mathrm{G}$ cap structure and a poly $(\mathrm{A})^{+}$tail. The binding of the $\mathrm{Sm}_{7}$ Ring complex to its $\mathrm{Sm}$ binding site triggers the hypermethylation of the cap structure by Tgs1. Besides, the $\mathrm{Sm}_{7}$ binding also leads to a 3'-end trimming performed through the combination of the Nrd1-Nab3 complex, the nuclear exosome and probably the TRAMP complex. After maturation, TLC1 is exported to the cytoplasm via the combination of the Crm/Xpo1 and mRNA export factors. In the cytoplasm, TLC1 assembles the telomerase components to form the telomerase complex. Telomerase is re-imported into the nucleus by Mtr10, which might also transport the factors necessary for TLC1 maturation. Finally, in the nucleus, telomerase elongates the shortened telomeres to maintain their length and to avoid senescence. 


\section{Discussion and Perspective}

\subsection{The life cycle of the telomerase complex}

The life cycle of the telomerase complex begins with the synthesis of the telomerase RNA TLC1. TLC1 is generated by RNA polymerase II and polyadenylated like other RNA polymerase II products (reviewed in (Gallardo and Chartrand, 2008). However, the association of the $\mathrm{Sm}_{7}$ complex to its binding site on the $3^{\prime}$ end leads to different processing events than those of the mRNAs (Seto et al., 1999). This $\mathrm{Sm}_{7}$ ring complex binding triggers two events: Hypermethylation of the 5' cap structure of TLC1 (Franke et al., 2008) and shortening of its 3' part through the Nab1-Nab3-Sen1 pathway (Noel et al., 2012). Our results have shown that mature $T L C 1$ accumulates in the nucleus upon an export block, suggesting that the complete maturation of the TLC1 RNA occurs in the nucleus. Nevertheless, the sequential order of these two events remains unclear. The only deduction that can be made is that they do not occur simultaneously, since the hypermethylation factor, Tgs1, is located in the nucleolus (Mouaikel et al., 2002) while Nrd1 and Nab3 are located in the nucleus in Saccharomyces cerevisiae (Huh et al., 2003). Moreover, whether these two events are dependent on each other is also unknown. Measurement of the processing of TLC1 with qRT-PCR in tgs $1 \triangle$ stains would help to answer this question. The $3^{\prime}$ processing of $T L C 1$ is performed through a pathway that contains a recognition system, which includes the Nab1-Nab3-Sen1 termination complex (Noel et al., 2012), and a degradation system, which contains the nuclear exosome (Coy et al., 2013). The boundary of the degradation is defined by the $\mathrm{Sm}_{7}$ ring binding site (Coy et al., 2013). Although Dis3 is the only catalytic core component of the Exo-10, the remaining components in the Exo-9, are required to recognise the substrate (Das and Das, 2013). Which exact component is involved in this TLC1 recognition is another interesting topic. Furthermore, the TRAMP complex component knockout strains show TLC1 processing defects, suggesting that they might be involved in TLC1 processing. This is consistent with the findings that the TRAMP complex interacts with the Nab1-Nab3-Sen1 pathway and is involved in the maturation and degradation of non-coding RNAs (Grzechnik 
and Kufel, 2008; Tudek et al., 2014).

The nuclear export of TLC1 is initiated after full maturation. TLC1 export is mediated by the Crm1/Xpo1 pathway (Gallardo et al., 2008), and our results demonstrate that this nuclear export additionally requires the mRNA export pathway. Such coordination has also been reported in the transport of ribosomal subunits (Faza et al., 2012; Yao et al., 2007). Since Xpo1 interacts with proteins and needs adaptors to contact the RNA (reviewed in (Doye, 2014)), such an adaptor for TLC1 nuclear export is unknown yet.

Upon arrival of mature $T L C 1$ in the cytoplasm, its secondary structure is recognised by the telomerase components. However, the serial events in formation of the telomerase holoenzyme remain unclear. A previous report has shown that the Est1-TLC1 interaction is Est2 and Est3 independent and the nuclear localisation of Est1 is mediated by Srp1, a karyopherin alpha (Hawkins and Friedman, 2014). This is different for TLC1, which is transported by Mtr10 (Ferrezuelo et al., 2002; Gallardo et al., 2008). It is possible that Est1 and Est2-Est3-TLC1 might be imported into the nucleus separately and are subsequently assembled to form the telomerase holoenzyme. This hypothesis is supported by the observation that in the early G1 phase of the cell cycle Yku80 recruits the Est2-TLC1 premature telomerase without Est1 on the subtelomeric region (Fisher et al., 2004). However, Est2 is in this context inactive and does not elongate the telomeres (Fischer et al., 2004), suggesting that this could only be a way for retaining the telomerase components in the nucleus. Our result showing that both Est1 and Est2 mislocailse to the cytoplasm in absence of TLC1 argues against this hypothesis and rather supports a model that formation of the telomerase holoenzyme might occur prior to the nuclear import. Certainly, there would be another possibility to explain that the Mtr10-independent nuclear localisation of Est1 is dependent on TLC1. Although Est1 is imported by Srp1, it needs bind to TLC1 for its nuclear retention. This hypothesis is similar to our previous proposal that the Est2-TLC1 complexes are anchored at chromosome ends in the G1 phase (Fischer et al., 2004) for their nuclear retention. The hypothesis can be examined by nucleo-cytoplasmic cell fractionation experiments. Two biogenesis models of telomerase have been proposed: 1) the shuttling model, in which TLC1 would be transported to the cytoplasm to be assembled to the 
telomerase with the telomerase components and is then re-imported into the nucleus; 2) the processing enzyme model, in which the necessary factors that are required for the maturation of TLC1 and the telomerase complex are transported by Mtr10 (Ferrezuelo et al., 2002). Our results suggest a hypothesis combining these two models. On the one side, TLC1 would be exported to the cytoplasm and form the telomerase complex with the protein components there, followed by its re-import into the nucleus via Mtr10. On the other side, the maturation of TLC1 requires processing factors (the TRAMP complex, the nuclear exosome), some of which are imported into the nucleus by Mtr10.

Telomerase defects lead to telomere shortening. So far, the genome wide screens that identified factors involved in telomere shortening mostly identified non-essential genes (Askree et al., 2004). Here we have shown that under a particular condition, e.g. at semi-permissive temperature, the mutants of essential genes could also lead to telomere shortening. This result largely expands our view of the factors involved in telomere maintenance. A similar observation has also been reported recently that at the semi-permissive temperature a SRP1 mutant, which encodes a defective karyopherin alpha, shows telomere shortening due to a failed nuclear import of Est1 (Hawkins and Friedman, 2014). These observations suggest that the senescence phenotypes could also be caused by defects of essential genes and there might be more factors that are involved in telomere maintenance than expected.

After elongation of the telomeres, it is still unclear how telomerase would be recycled. Telomerase RNA is one of the most stable transcripts in a given organism, e.g. the half-life of around 4 weeks in $\mathrm{H} 1299$ cells, which is currently the RNA with the longest lifetime identified so far (Bodnar et al., 1998; Yi et al., 1999). In Saccharomyces cerevisiae, the half-life of TLC1 is longer than 60 minutes (Chapon et al., 1997). How the organism protects TLC1 from the access of the degradation machinery is unclear. One explanation might be that since TLC1 is the factor with the lowest abundance of all telomerase components (Mozdy and Cech, 2006), all RNA molecules are integrated into the telomerase complex and thus protected from degradation by the bound proteins. 


\subsection{RNA nuclear export}

As identified in this study, TLC1 utilises Xpo1/Crm1 and Mex67 for its export to the cytosol. However, why both factors contribute to the export remains unknown. Interestingly, Mex67 is also involved in transporting pre-60S (Yao et al., 2007) and pre-40S ribosomes (Faza et al., 2012) together with Xpo1/Crm1 (Ho et al., 2000; Johnson et al., 2002; Moy and Silver, 1999; Nissan et al., 2002). These observations suggest that cooperative use of $\mathrm{Xpo1/Crm1} \mathrm{and}$ Mex67 could be one of the ways for transporting certain RNAs.

For most spliceosomal snRNAs, it has been shown that in human cells they are transported into the cytoplasm for certain maturation steps including the binding of the Sm ring complex, 5' cap hypermethylation, as well as $3^{\prime}$ trimming (reviewed in (Will and Luhrmann, 2001)). Nevertheless, in yeast these maturation steps mainly occur in the nucleus. However, yeast $T L C 1$, as a non-coding RNA, requires the journey to the cytoplasm for the formation of the telomerase. In contrast, snRNAs are integrated into the spliceosomes in the nucleus (reviewed in (Will and Luhrmann, 2001)). Interestingly, a nucleo-cytoplasmic shuttling of some snRNAs and snoRNAs have nevertheless been observed in budding yeast by using heterokaryon assays, which has been suggested to be due to a global leakage or a molecular exchange between nuclei in this special cell type (Olson and Siliciano, 2003). Therefore, it will require further experiments to find out if these non-coding RNAs shuttle in yeast. Moreover, if this is the case, whether the nuclear export of the RNAs is also mediated by cooperation of Xpo1/Crm1 and Mex67 remains to be known. 


\subsection{Mtr10 and its cargoes}

Mtr10 has been first identified in a screen of $\underline{m} R N A$ transport-defective ( $m t r)$ mutants (Kadowaki et al., 1994). Later investigations have shown that the mRNA transport defects in the mtr10 mutant are probably not due to loss of the function of Mtr10 directly, but rather indirectly by mediating the nuclear re-import of the three mRNA adaptor proteins for Mex67-Mtr2, Hrb1, Gbp2 and Npl3 (Pemberton et al., 1997; Senger et al., 1998; Windgassen and Krebber, 2003). Here we have identified that, in addition to these mRNA-binding proteins, Mtr10 might transport the TRAMP complex components, Mtr4 and Trf4, and the exosome component, Dis3 (Lea Steffen and Heike Krebber), into the nucleus. These findings are also supported by the immunoprecipitation experiments that show a physical interaction between Mtr10 and these factors. Interestingly, several global analyses of protein complexes have uncovered the interaction of Srp1, a karyopherin alpha, with all of the exosome core components that supported a possible transport function for Srp1 (Collins et al., 2007; Gavin et al., 2002; Ho et al., 2002; Krogan et al., 2006; Peng et al., 2003; Synowsky et al., 2009). It still remains to be shown if indeed Srp1 participate in the Mtr10 mediated nuclear import of these factors. Interestingly, the telomerase component Est1 might be transported via Srp1 (Hawkins and Friedman, 2014) but another telomerase component TLC1 is imported via Mtr10. Therefore, further experiments, including the investigation of a physical interaction and genetic interaction between Mtr10 and Srp1, would be necessary to verify this potential cooperation. In fact, although the deletion of MTR10 is lethal at higher temperatures (e.g. $\left.37^{\circ} \mathrm{C}\right)$, it shows only a strong growth defect at lower growth temperatures $\left(23-30^{\circ} \mathrm{C}\right)$ (Pemberton et al., 1997; Senger et al., 1998). This suggests that although Mtr10 is an important karyopherin, it is not essential. Without Mtr10 its substrates are still transported, however with much lower efficiency. This is further supported by the observation that overexpression of TLC1 in mtr10A cells leads to a rescue of the telomere shortening (Ferrezuelo et al., 2002).

Moreover, it is unclear if Mtr10 imports the exosome complex and the TRAMP complex components as single proteins or as a formed complex. The can be addressed by interaction 
studies in mtr10 mutants. 


\section{References}

Abou Elela, S., and Ares, M., Jr. (1998). Depletion of yeast RNase III blocks correct U2 3' end formation and results in polyadenylated but functional U2 snRNA. The EMBO journal 17, 3738-3746.

Allmang, C., Mitchell, P., Petfalski, E., and Tollervey, D. (2000). Degradation of ribosomal RNA precursors by the exosome. Nucleic Acids Res 28, 1684-1691.

Arigo, J.T., Eyler, D.E., Carroll, K.L., and Corden, J.L. (2006). Termination of cryptic unstable transcripts is directed by yeast RNA-binding proteins Nrd1 and Nab3. Mol Cell 23, 841-851.

AskjaerStade, K., Ford, C.S., Guthrie, C., and Weis, K. (1997). Exportin 1 (Crm1p) is an essential nuclear export factor. Cell 90, 1041-1050.

Askree, S.H., Yehuda, T., Smolikov, S., Gurevich, R., Hawk, J., Coker, C., Krauskopf, A., Kupiec, M., and McEachern, M.J. (2004). A genome-wide screen for Saccharomyces cerevisiae deletion mutants that affect telomere length. Proceedings of the National Academy of Sciences of the United States of America 101, 8658-8663.

Bachi, A., Braun, I.C., Rodrigues, J.P., Pante, N., Ribbeck, K., von Kobbe, C., Kutay, U., Wilm, M., Gorlich, D., Carmo-Fonseca, M., et al. (2000). The C-terminal domain of TAP interacts with the nuclear pore complex and promotes export of specific CTE-bearing RNA substrates. RNA 6, 136-158.

Bianchi, A., and Shore, D. (2007). Increased association of telomerase with short telomeres in yeast. Genes Dev 21, 1726-1730.

Bodnar, A.G., Ouellette, M., Frolkis, M., Holt, S.E., Chiu, C.P., Morin, G.B., Harley, C.B., Shay, J.W., Lichtsteiner, S., and Wright, W.E. (1998). Extension of life-span by introduction of telomerase into normal human cells. Science 279, 349-352.

Bonetti, D., Clerici, M., Anbalagan, S., Martina, M., Lucchini, G., and Longhese, M.P. (2010a). Shelterin-like proteins and $\mathrm{Yku}$ inhibit nucleolytic processing of Saccharomyces cerevisiae telomeres. Plos Genet 6, e1000966.

Bonetti, D., Clerici, M., Manfrini, N., Lucchini, G., and Longhese, M.P. (2010b). The MRX complex plays multiple functions in resection of Yku- and Rif2-protected DNA ends. Plos One 5, e14142.

Bosoy, D., Peng, Y., Mian, I.S., and Lue, N.F. (2003). Conserved N-terminal motifs of telomerase reverse transcriptase required for ribonucleoprotein assembly in vivo. The Journal of biological chemistry 278, 3882-3890.

Boulton, S.J., and Jackson, S.P. (1998). Components of the Ku-dependent non-homologous end-joining pathway are involved in telomeric length maintenance and telomeric silencing. The EMBO journal 17, 1819-1828.

Bourns, B.D., Alexander, M.K., Smith, A.M., and Zakian, V.A. (1998). Sir proteins, Rif proteins, and Cdc13p bind Saccharomyces telomeres in vivo. Molecular and cellular biology 18, 5600-5608.

Bousquet-Antonelli, C., Presutti, C., and Tollervey, D. (2000). Identification of a regulated pathway for nuclear pre-mRNA turnover. Cell 102, 765-775.

Box, J.A., Bunch, J.T., Tang, W., and Baumann, P. (2008). Spliceosomal cleavage generates the 3' end of telomerase RNA. Nature 456, 910-914.

Brune, C., Munchel, S.E., Fischer, N., Podtelejnikov, A.V., and Weis, K. (2005). Yeast poly(A)-binding protein Pab1 shuttles between the nucleus and the cytoplasm and functions in mRNA export. Rna-a Publication of the Rna Society 11, 517-531. 
Burkard, K.T., and Butler, J.S. (2000). A nuclear 3'-5' exonuclease involved in mRNA degradation interacts with Poly $(\mathrm{A})$ polymerase and the hnRNA protein Npl3p. Molecular and cellular biology 20, 604-616.

Carroll, K.L., Pradhan, D.A., Granek, J.A., Clarke, N.D., and Corden, J.L. (2004). Identification of cis elements directing termination of yeast nonpolyadenylated snoRNA transcripts. Molecular and cellular biology 24, 6241-6252.

Chan, A., Boule, J.B., and Zakian, V.A. (2008). Two pathways recruit telomerase to Saccharomyces cerevisiae telomeres. Plos Genet 4, e1000236.

Chan, C.S., and Tye, B.K. (1983). Organization of DNA sequences and replication origins at yeast telomeres. Cell 33, 563-573.

Chapon, C., Cech, T.R., and Zaug, A.J. (1997). Polyadenylation of telomerase RNA in budding yeast. Rna-a Publication of the Rna Society 3, 1337-1351.

Cohn, M., and Blackburn, E.H. (1995). Telomerase in yeast. Science 269, 396-400.

Collins, S.R., Kemmeren, P., Zhao, X.C., Greenblatt, J.F., Spencer, F., Holstege, F.C., Weissman, J.S., and Krogan, N.J. (2007). Toward a comprehensive atlas of the physical interactome of Saccharomyces cerevisiae. Molecular \& cellular proteomics : MCP 6, 439-450.

Conrad, M.N., Wright, J.H., Wolf, A.J., and Zakian, V.A. (1990). RAP1 protein interacts with yeast telomeres in vivo: overproduction alters telomere structure and decreases chromosome stability. Cell 63, 739-750.

Coy, S., Volanakis, A., Shah, S., and Vasiljeva, L. (2013). The Sm Complex Is Required for the Processing of Non-Coding RNAs by the Exosome. Plos One 8.

Creamer, T.J., Darby, M.M., Jamonnak, N., Schaughency, P., Hao, H., Wheelan, S.J., and Corden, J.L. (2011). Transcriptome-wide binding sites for components of the Saccharomyces cerevisiae non-poly(A) termination pathway: Nrd1, Nab3, and Sen1. Plos Genet 7, e1002329.

Dandjinou, A.T., Levesque, N., Larose, S., Lucier, J.F., Abou Elela, S., and Wellinger, R.J. (2004). A phylogenetically based secondary structure for the yeast telomerase RNA. Current biology : CB 14, 1148-1158.

Das, S., and Das, B. (2013). mRNA quality control pathways in Saccharomyces cerevisiae. Journal of biosciences 38, 615-640.

de la Cruz, J., Kressler, D., and Linder, P. (1999). Unwinding RNA in Saccharomyces cerevisiae: DEAD-box proteins and related families. Trends in biochemical sciences 24, 192-198.

de la Cruz, J., Kressler, D., Tollervey, D., and Linder, P. (1998). Dob1p (Mtr4p) is a putative ATP-dependent RNA helicase required for the $3^{\prime}$ end formation of 5.8S rRNA in Saccharomyces cerevisiae. The EMBO journal 17, 1128-1140.

de Vegvar, H.E., Lund, E., and Dahlberg, J.E. (1986). 3' end formation of U1 snRNA precursors is coupled to transcription from snRNA promoters. Cell 47, 259-266.

Del Priore, V., Heath, C., Snay, C., MacMillan, A., Gorsch, L., Dagher, S., and Cole, C. (1997). A structure/function analysis of Rat7p/Nup159p, an essential nucleoporin of Saccharomyces cerevisiae. Journal of cell science 110 ( Pt 23), 2987-2999.

Dewar, J.M., and Lydall, D. (2012). Similarities and differences between "uncapped" telomeres and DNA double-strand breaks. Chromosoma 121, 117-130.

Dez, C., Dlakic, M., and Tollervey, D. (2007). Roles of the HEAT repeat proteins Utp10 and Utp20 in $40 \mathrm{~S}$ ribosome maturation. Rna-a Publication of the Rna Society 13, 1516-1527.

DeZwaan, D.C., and Freeman, B.C. (2009). The conserved Est1 protein stimulates telomerase DNA 
extension activity. Proceedings of the National Academy of Sciences of the United States of America 106, 17337-17342.

Doye, V. (2014). Preface. Nuclear pore complexes and nucleocytoplasmic transport-methods. Methods in cell biology 122 , xix-xx.

Eckmann, C.R., Rammelt, C., and Wahle, E. (2011). Control of poly(A) tail length. Wiley interdisciplinary reviews. RNA 2, 348-361.

Egecioglu, D.E., Henras, A.K., and Chanfreau, G.F. (2006). Contributions of Trf4p- and Trf5p-dependent polyadenylation to the processing and degradative functions of the yeast nuclear exosome. Rna-a Publication of the Rna Society 12, 26-32.

Evans, S.K., and Lundblad, V. (1999). Est1 and Cdc13 as comediators of telomerase access. Science 286 , 117-120.

Fasken, M.B., Leung, S.W., Banerjee, A., Kodani, M.O., Chavez, R., Bowman, E.A., Purohit, M.K., Rubinson, M.E., Rubinson, E.H., and Corbett, A.H. (2011). Air1 zinc knuckles 4 and 5 and a conserved IWRXY motif are critical for the function and integrity of the Trf4/5-Air1/2-Mtr4 polyadenylation (TRAMP) RNA quality control complex. The Journal of biological chemistry 286, 37429-37445.

Faza, M.B., Chang, Y., Occhipinti, L., Kemmler, S., and Panse, V.G. (2012). Role of Mex67-Mtr2 in the Nuclear Export of 40S Pre-Ribosomes. Plos Genet 8.

Ferrezuelo, F., Steiner, B., Aldea, M., and Futcher, B. (2002). Biogenesis of yeast telomerase depends on the importin mtr10. Molecular and cellular biology 22,6046-6055.

Fiorentini, P., Huang, K.N., Tishkoff, D.X., Kolodner, R.D., and Symington, L.S. (1997). Exonuclease I of Saccharomyces cerevisiae functions in mitotic recombination in vivo and in vitro. Molecular and cellular biology 17, 2764-2773.

Fischer, T., Rodriguez-Navarro, S., Pereira, G., Racz, A., Schiebel, E., and Hurt, E. (2004). Yeast centrin Cdc31 is linked to the nuclear mRNA export machinery. Nature cell biology 6, 840-848.

Fisher, T.S., Taggart, A.K., and Zakian, V.A. (2004). Cell cycle-dependent regulation of yeast telomerase by Ku. Nat Struct Mol Biol 11, 1198-1205.

Forstemann, K., and Lingner, J. (2001). Molecular basis for telomere repeat divergence in budding yeast. Molecular and cellular biology 21, 7277-7286.

Franke, J., Gehlen, J., and Ehrenhofer-Murray, A.E. (2008). Hypermethylation of yeast telomerase RNA by the snRNA and snoRNA methyltransferase Tgs1. Journal of cell science 121, 3553-3560.

Friedman, K.L., and Cech, T.R. (1999). Essential functions of amino-terminal domains in the yeast telomerase catalytic subunit revealed by selection for viable mutants. Genes Dev 13, 2863-2874.

Friedman, K.L., Heit, J.J., Long, D.M., and Cech, T.R. (2003). N-terminal domain of yeast telomerase reverse transcriptase: recruitment of Est3p to the telomerase complex. Molecular biology of the cell 14, 1-13.

Gadal, O., Strauss, D., Kessl, J., Trumpower, B., Tollervey, D., and Hurt, E. (2001). Nuclear export of 60s ribosomal subunits depends on Xpo1p and requires a nuclear export sequence-containing factor, Nmd3p, that associates with the large subunit protein Rpl10p. Molecular and cellular biology 21, 3405-3415.

Gallardo, F., and Chartrand, P. (2008). Telomerase biogenesis. Rna Biol 5, 212-215.

Gallardo, F., Laterreur, N., Cusanelli, E., Ouenzar, F., Querido, E., Wellinger, R.J., and Chartrand, P. (2011). Live cell imaging of telomerase RNA dynamics reveals cell cycle-dependent clustering of telomerase at elongating telomeres. Mol Cell 44, 819-827. 
Gallardo, F., Olivier, C., Dandjinou, A.T., Wellinger, R.J., and Chartrand, P. (2008). TLC1 RNA nucleo-cytoplasmic trafficking links telomerase biogenesis to its recruitment to telomeres. Embo J 27, 748-757.

Gauss, R., Trautwein, M., Sommer, T., and Spang, A. (2005). New modules for the repeated internal and N-terminal epitope tagging of genes in Saccharomyces cerevisiae. Yeast 22, 1-12.

Gavin, A.C., Bosche, M., Krause, R., Grandi, P., Marzioch, M., Bauer, A., Schultz, J., Rick, J.M., Michon, A.M., Cruciat, C.M., et al. (2002). Functional organization of the yeast proteome by systematic analysis of protein complexes. Nature 415, 141-147.

Gilson, E., Roberge, M., Giraldo, R., Rhodes, D., and Gasser, S.M. (1993). Distortion of the DNA double helix by RAP1 at silencers and multiple telomeric binding sites. Journal of molecular biology 231, 293-310.

Gorsch, L.C., Dockendorff, T.C., and Cole, C.N. (1995). A conditional allele of the novel repeat-containing yeast nucleoporin RAT7/NUP159 causes both rapid cessation of mRNA export and reversible clustering of nuclear pore complexes. The Journal of cell biology 129, 939-955.

Gottschling, D.E., Aparicio, O.M., Billington, B.L., and Zakian, V.A. (1990). Position effect at S. cerevisiae telomeres: reversible repression of Pol II transcription. Cell 63, 751-762.

Grant, R.P., Hurt, E., Neuhaus, D., and Stewart, M. (2002). Structure of the C-terminal FG-nucleoporin binding domain of Tap/NXF1. Nature structural biology 9, 247-251.

Gravel, S., Larrivee, M., Labrecque, P., and Wellinger, R.J. (1998). Yeast Ku as a regulator of chromosomal DNA end structure. Science 280, 741-744.

Grzechnik, P., and Kufel, J. (2008). Polyadenylation linked to transcription termination directs the processing of snoRNA precursors in yeast. Mol Cell 32, 247-258.

Gudipati, R.K., Xu, Z., Lebreton, A., Seraphin, B., Steinmetz, L.M., Jacquier, A., and Libri, D. (2012). Extensive degradation of RNA precursors by the exosome in wild-type cells. Mol Cell 48, 409-421.

Gwizdek, C., Iglesias, N., Rodriguez, M.S., Ossareh-Nazari, B., Hobeika, M., Divita, G., Stutz, F., and Dargemont, C. (2006). Ubiquitin-associated domain of Mex67 synchronizes recruitment of the mRNA export machinery with transcription. Proceedings of the National Academy of Sciences of the United States of America 103, 16376-16381.

Hamill, S., Wolin, S.L., and Reinisch, K.M. (2010). Structure and function of the polymerase core of TRAMP, a RNA surveillance complex. Proceedings of the National Academy of Sciences of the United States of America 107, 15045-15050.

Hamm, J., and Mattaj, I.W. (1990). Monomethylated cap structures facilitate RNA export from the nucleus. Cell 63, 109-118.

Hardy, C.F., Balderes, D., and Shore, D. (1992a). Dissection of a carboxy-terminal region of the yeast regulatory protein RAP1 with effects on both transcriptional activation and silencing. Molecular and cellular biology 12, 1209-1217.

Hardy, C.F., Sussel, L., and Shore, D. (1992b). A RAP1-interacting protein involved in transcriptional silencing and telomere length regulation. Genes Dev 6, 801-814.

Harley, C.B., Futcher, A.B., and Greider, C.W. (1990). Telomeres shorten during ageing of human fibroblasts. Nature 345, 458-460.

Hawkins, C., and Friedman, K.L. (2014). Normal Telomere Length Maintenance in Yeast Requires Nuclear Import of the Ever Shorter Telomeres 1 (Est1) Protein via the Importin Alpha Pathway. Eukaryotic cell.

Hayflick, L. (1979). Cell biology of aging. Fed Proc 38, 1847-1850. 
Hector, R.E., Shtofman, R.L., Ray, A., Chen, B.R., Nyun, T., Berkner, K.L., and Runge, K.W. (2007). Tel1p preferentially associates with short telomeres to stimulate their elongation. Mol Cell 27, 851-858. Hernandez, N., and Weiner, A.M. (1986). Formation of the 3' end of U1 snRNA requires compatible snRNA promoter elements. Cell 47, 249-258.

Ho, J.H., Kallstrom, G., and Johnson, A.W. (2000). Nmd3p is a Crm1p-dependent adapter protein for nuclear export of the large ribosomal subunit. The Journal of cell biology 151, 1057-1066.

Ho, Y., Gruhler, A., Heilbut, A., Bader, G.D., Moore, L., Adams, S.L., Millar, A., Taylor, P., Bennett, K., Boutilier, K., et al. (2002). Systematic identification of protein complexes in Saccharomyces cerevisiae by mass spectrometry. Nature 415, 180-183.

Hobeika, M., Brockmann, C., Gruessing, F., Neuhaus, D., Divita, G., Stewart, M., and Dargemont, C. (2009). Structural requirements for the ubiquitin-associated domain of the mRNA export factor Mex67 to bind its specific targets, the transcription elongation THO complex component Hpr1 and nucleoporin FXFG repeats. The Journal of biological chemistry 284, 17575-17583.

Hodge, C.A., Colot, H.V., Stafford, P., and Cole, C.N. (1999). Rat8p/Dbp5p is a shuttling transport factor that interacts with Rat7p/Nup159p and Gle1p and suppresses the mRNA export defect of xpo1-1 cells. The EMBO journal 18, 5778-5788.

Holub, P., Lalakova, J., Cerna, H., Pasulka, J., Sarazova, M., Hrazdilova, K., Arce, M.S., Hobor, F., Stefl, R., and Vanacova, S. (2012). Air2p is critical for the assembly and RNA-binding of the TRAMP complex and the KOW domain of Mtr4p is crucial for exosome activation. Nucleic acids research 40, 5679-5693.

Hopper, A.K. (2006). Cellular dynamics of small RNAs. Critical reviews in biochemistry and molecular biology 41, 3-19.

Houseley, J., and Tollervey, D. (2006). Yeast Trf5p is a nuclear poly(A) polymerase. Embo Rep 7, 205-211.

Hughes, T.R., Evans, S.K., Weilbaecher, R.G., and Lundblad, V. (2000a). The Est3 protein is a subunit of yeast telomerase. Current biology : CB 10, 809-812.

Hughes, T.R., Weilbaecher, R.G., Walterscheid, M., and Lundblad, V. (2000b). Identification of the single-strand telomeric DNA binding domain of the Saccharomyces cerevisiae Cdc13 protein. Proceedings of the National Academy of Sciences of the United States of America 97, 6457-6462.

Huh, W.K., Falvo, J.V., Gerke, L.C., Carroll, A.S., Howson, R.W., Weissman, J.S., and O'Shea, E.K. (2003). Global analysis of protein localization in budding yeast. Nature 425, 686-691.

Hurt, E., Hannus, S., Schmelzl, B., Lau, D., Tollervey, D., and Simos, G. (1999). A novel in vivo assay reveals inhibition of ribosomal nuclear export in ran-cycle and nucleoporin mutants. The Journal of cell biology 144, 389-401.

Izaurralde, E., Lewis, J., Gamberi, C., Jarmolowski, A., McGuigan, C., and Mattaj, I.W. (1995). A cap-binding protein complex mediating $U$ snRNA export. Nature 376, 709-712.

Jackson, R.N., Klauer, A.A., Hintze, B.J., Robinson, H., van Hoof, A., and Johnson, S.J. (2010). The crystal structure of Mtr4 reveals a novel arch domain required for rRNA processing. The EMBO journal 29, 2205-2216.

Jia, H., Wang, X., Liu, F., Guenther, U.P., Srinivasan, S., Anderson, J.T., and Jankowsky, E. (2011). The RNA helicase Mtr4p modulates polyadenylation in the TRAMP complex. Cell 145, 890-901.

Johnson, A.W., Lund, E., and Dahlberg, J. (2002). Nuclear export of ribosomal subunits. Trends in biochemical sciences $27,580-585$.

Kadaba, S., Krueger, A., Trice, T., Krecic, A.M., Hinnebusch, A.G., and Anderson, J. (2004). Nuclear 
surveillance and degradation of hypomodified initiator tRNA(Met) in S-cerevisiae. Gene Dev 18, 1227-1240.

Kadaba, S., Wang, X.Y., and Anderson, J.T. (2006). Nuclear RNA surveillance in Saccharomyces cerevisiae: Trf4p-dependent polyadenylation of nascent hypomethylated tRNA and an aberrant form of 5 S rRNA. Rna-a Publication of the Rna Society 12, 508-521.

Kadowaki, T., Chen, S., Hitomi, M., Jacobs, E., Kumagai, C., Liang, S., Schneiter, R., Singleton, D., Wisniewska, J., and Tartakoff, A.M. (1994). Isolation and characterization of Saccharomyces cerevisiae mRNA transport-defective (mtr) mutants. The Journal of cell biology 126, 649-659.

Kang, Y., and Cullen, B.R. (1999). The human Tap protein is a nuclear mRNA export factor that contains novel RNA-binding and nucleocytoplasmic transport sequences. Genes Dev 13, 1126-1139.

Katahira, J., Strasser, K., Podtelejnikov, A., Mann, M., Jung, J.U., and Hurt, E. (1999). The Mex67p-mediated nuclear mRNA export pathway is conserved from yeast to human. The EMBO journal 18, 2593-2609.

Kironmai, K.M., and Muniyappa, K. (1997). Alteration of telomeric sequences and senescence caused by mutations in RAD50 of Saccharomyces cerevisiae. Genes to cells : devoted to molecular \& cellular mechanisms 2, 443-455.

Knop, M., Siegers, K., Pereira, G., Zachariae, W., Winsor, B., Nasmyth, K., and Schiebel, E. (1999). Epitope tagging of yeast genes using a PCR-based strategy: More tags and improved practical routines. Yeast $15,963-972$.

Kohler, A., and Hurt, E. (2007). Exporting RNA from the nucleus to the cytoplasm. Nat Rev Mol Cell Bio 8, 761-773.

Krogan, N.J., Cagney, G., Yu, H., Zhong, G., Guo, X., Ignatchenko, A., Li, J., Pu, S., Datta, N., Tikuisis, A.P., et al. (2006). Global landscape of protein complexes in the yeast Saccharomyces cerevisiae. Nature 440, 637-643.

Kufel, J., Bousquet-Antonelli, C., Beggs, J.D., and Tollervey, D. (2004). Nuclear pre-mRNA decapping and $5^{\prime}$ degradation in yeast require the Lsm2-8p complex. Molecular and cellular biology 24, 9646-9657.

Kupiec, M. (2014). Biology of telomeres: lessons from budding yeast. FEMS microbiology reviews 38, 144-171.

LaCava, J., Houseley, J., Saveanu, C., Petfalski, E., Thompson, E., Jacquier, A., and Tollervey, D. (2005). RNA degradation by the exosome is promoted by a nuclear polyade nylation complex. Cell 121, 713-724.

Larrivee, M., LeBel, C., and Wellinger, R.J. (2004). The generation of proper constitutive G-tails on yeast telomeres is dependent on the MRX complex. Genes Dev 18, 1391-1396.

Libri, D., Dower, K., Boulay, J., Thomsen, R., Rosbash, M., and Jensen, T.H. (2002). Interactions between mRNA export commitment, 3'-end quality control, and nuclear degradation. Molecular and cellular biology 22, 8254-8266.

Liker, E., Fernandez, E., Izaurralde, E., and Conti, E. (2000). The structure of the mRNA export factor TAP reveals a cis arrangement of a non-canonical RNP domain and an LRR domain. The EMBO journal 19, 5587-5598.

Lin, J., Ly, H., Hussain, A., Abraham, M., Pearl, S., Tzfati, Y., Parslow, T.G., and Blackburn, E.H. (2004). A universal telomerase RNA core structure includes structured motifs required for binding the telomerase reverse transcriptase protein. Proceedings of the National Academy of Sciences of the United States of America 101, 14713-14718. 
Lin, J.J., and Zakian, V.A. (1996). The Saccharomyces CDC13 protein is a single-strand TG1-3 telomeric DNA-binding protein in vitro that affects telomere behavior in vivo. Proceedings of the National Academy of Sciences of the United States of America 93, 13760-13765.

Lingner, J., Cooper, J.P., and Cech, T.R. (1995). Telomerase and DNA end replication: no longer a lagging strand problem? Science 269, 1533-1534.

Lingner, J., Hughes, T.R., Shevchenko, A., Mann, M., Lundblad, V., and Cech, T.R. (1997). Reverse transcriptase motifs in the catalytic subunit of telomerase. Science 276, 561-567.

Liu, Q., Greimann, J.C., and Lima, C.D. (2006). Reconstitution, activities, and structure of the eukaryotic RNA exosome. Cell 127, 1223-1237.

Liu, Y., Guo, W., Tartakoff, P.Y., and Tartakoff, A.M. (1999). A Crm1p-independent nuclear export path for the mRNA-associated protein, Npl3p/Mtr13p. Proceedings of the National Academy of Sciences of the United States of America 96, 6739-6744.

Livengood, A.J., Zaug, A.J., and Cech, T.R. (2002). Essential regions of Saccharomyces cerevisiae telomerase RNA: separate elements for Est1p and Est2p interaction. Molecular and cellular biology 22, 2366-2374.

Louis, E.J., Naumova, E.S., Lee, A., Naumov, G., and Haber, J.E. (1994). The chromosome end in yeast: its mosaic nature and influence on recombinational dynamics. Genetics 136, 789-802.

Lubin, J.W., Tucey, T.M., and Lundblad, V. (2012). The interaction between the yeast telomerase RNA and the Est1 protein requires three structural elements. Rna-a Publication of the Rna Society 18, 1597-1604.

Lundblad, V., and Szostak, J.W. (1989). A Mutant with a Defect in Telomere Elongation Leads to Senescence in Yeast. Cell 57, 633-643.

Lustig, A.J., Kurtz, S., and Shore, D. (1990). Involvement of the silencer and UAS binding protein RAP1 in regulation of telomere length. Science 250, 549-553.

Mantiero, D., Clerici, M., Lucchini, G., and Longhese, M.P. (2007). Dual role for Saccharomyces cerevisiae Tel1 in the checkpoint response to double-strand breaks. Embo Rep 8, 380-387.

Martin, S.G., Laroche, T., Suka, N., Grunstein, M., and Gasser, S.M. (1999). Relocalization of telomeric $\mathrm{Ku}$ and SIR proteins in response to DNA strand breaks in yeast. Cell 97, 621-633.

Matera, A.G., Terns, R.M., and Terns, M.P. (2007). Non-coding RNAs: lessons from the small nuclear and small nucleolar RNAs. Nature reviews. Molecular cell biology 8, 209-220.

Mitchell, P., Petfalski, E., Shevchenko, A., Mann, M., and Tollervey, D. (1997). The exosome: A conserved eukaryotic RNA processing complex containing multiple $3^{\prime}->5$ ' exoribonucleases. Cell 91, 457-466.

Mitton-Fry, R.M., Anderson, E.M., Hughes, T.R., Lundblad, V., and Wuttke, D.S. (2002). Conserved structure for single-stranded telomeric DNA recognition. Science 296, 145-147.

Mitton-Fry, R.M., Anderson, E.M., Theobald, D.L., Glustrom, L.W., and Wuttke, D.S. (2004). Structural basis for telomeric single-stranded DNA recognition by yeast Cdc13. Journal of molecular biology 338, 241-255.

Moretti, P., Freeman, K., Coodly, L., and Shore, D. (1994). Evidence that a complex of SIR proteins interacts with the silencer and telomere-binding protein RAP1. Genes Dev 8, 2257-2269.

Morlando, M., Greco, P., Dichtl, B., Fatica, A., Keller, W., and Bozzoni, I. (2002). Functional analysis of yeast snoRNA and snRNA 3 '-end formation mediated by uncoupling of cleavage and polyadenylation. Molecular and cellular biology 22, 1379-1389.

Morris, D.K., and Lundblad, V. (1997). Programmed translational frameshifting in a gene required for 
yeast telomere replication. Current biology : CB 7, 969-976.

Mouaikel, J., Verheggen, C., Bertrand, E., Tazi, J., and Bordonne, R. (2002). Hypermethylation of the cap structure of both yeast snRNAs and snoRNAs requires a conserved methyltransferase that is localized to the nucleolus. Mol Cell 9, 891-901.

Moy, T.I., and Silver, P.A. (1999). Nuclear export of the small ribosomal subunit requires the ran-GTPase cycle and certain nucleoporins. Genes Dev 13, 2118-2133.

Mozdy, A.D., and Cech, T.R. (2006). Low abundance of telomerase in yeast: implications for telomerase haploinsufficiency. RNA 12, 1721-1737.

Nakada, D., Matsumoto, K., and Sugimoto, K. (2003). ATM-related Tel1 associates with double-strand breaks through an Xrs2-dependent mechanism. Genes Dev 17, 1957-1962.

Neville, M., Stutz, F., Lee, L., Davis, L.I., and Rosbash, M. (1997). The importin-beta family member Crm1p bridges the interaction between Rev and the nuclear pore complex during nuclear export. Current biology : CB 7, 767-775.

Nissan, T.A., Bassler, J., Petfalski, E., Tollervey, D., and Hurt, E. (2002). 60 S pre-ribosome formation viewed from assembly in the nucleolus until export to the cytoplasm. The EMBO journal 21, 5539-5547.

Noel, J.F., Larose, S., Abou Elela, S., and Wellinger, R.J. (2012). Budding yeast telomerase RNA transcription termination is dictated by the Nrd1/Nab3 non-coding RNA termination pathway. Nucleic acids research 40, 5625-5636.

Nugent, C.I., Hughes, T.R., Lue, N.F., and Lundblad, V. (1996). Cdc13p: a single-strand telomeric DNA-binding protein with a dual role in yeast telomere maintenance. Science 274, 249-252.

Ohno, M., Segref, A., Bachi, A., Wilm, M., and Mattaj, I.W. (2000). PHAX, a mediator of U snRNA nuclear export whose activity is regulated by phosphorylation. Cell 101, 187-198.

Olovnikov, A.M. (1971). [Principle of marginotomy in template synthesis of polynucleotides]. Doklady Akademii nauk SSSR 201, 1496-1499.

Olson, B.L., and Siliciano, P.G. (2003). A diverse set of nuclear RNAs transfer between nuclei of yeast heterokaryons. Yeast 20, 893-903.

Osterhage, J.L., Talley, J.M., and Friedman, K.L. (2006). Proteasome-dependent degradation of Est1p regulates the cell cycle-restricted assembly of telomerase in Saccharomyces cerevisiae. Nat Struct Mol Biol 13, 720-728.

Palladino, F., Laroche, T., Gilson, E., Axelrod, A., Pillus, L., and Gasser, S.M. (1993). SIR3 and SIR4 proteins are required for the positioning and integrity of yeast telomeres. Cell 75, 543-555

Paolo, S.S., Vanacova, S., Schenk, L., Scherrer, T., Blank, D., Keller, W., and Gerber, A.P. (2009). Distinct Roles of Non-Canonical Poly(A) Polymerases in RNA Metabolism. Plos Genet 5.

Pemberton, L.F., Rosenblum, J.S., and Blobel, G. (1997). A distinct and parallel pathway for the nuclear import of an mRNA-binding protein. The Journal of cell biology 139, 1645-1653.

Peng, W.T., Robinson, M.D., Mnaimneh, S., Krogan, N.J., Cagney, G., Morris, Q., Davierwala, A.P., Grigull, J., Yang, X., Zhang, W., et al. (2003). A panoramic view of yeast noncoding RNA processing. Cell $113,919-933$.

Pennock, E., Buckley, K., and Lundblad, V. (2001). Cdc13 delivers separate complexes to the telomere for end protection and replication. Cell 104, 387-396.

Pfingsten, J.S., Goodrich, K.J., Taabazuing, C., Ouenzar, F., Chartrand, P., and Cech, T.R. (2012). Mutually exclusive binding of telomerase RNA and DNA by Ku alters telomerase recruitment model. Cell $148,922-932$ 
Porter, S.E., Greenwell, P.W., Ritchie, K.B., and Petes, T.D. (1996). The DNA-binding protein Hdf1p (a putative $\mathrm{Ku}$ homologue) is required for maintaining normal telomere length in Saccharomyces cerevisiae. Nucleic acids research 24, 582-585.

Pryde, F.E., and Louis, E.J. (1999). Limitations of silencing at native yeast telomeres. The EMBO journal 18, 2538-2550.

Puig, O., Caspary, F., Rigaut, G., Rutz, B., Bouveret, E., Bragado-Nilsson, E., Wilm, M., and Seraphin, B. (2001). The tandem affinity purification (TAP) method: a general procedure of protein complex purification. Methods 24, 218-229.

Qi, H., and Zakian, V.A. (2000). The Saccharomyces telomere-binding protein Cdc13p interacts with both the catalytic subunit of DNA polymerase alpha and the telomerase-associated est1 protein. Genes Dev 14, 1777-1788.

Qiao, F., and Cech, T.R. (2008). Triple-helix structure in telomerase RNA contributes to catalysis. Nat Struct Mol Biol 15, 634-640.

Raghuraman, M.K., Winzeler, E.A., Collingwood, D., Hunt, S., Wodicka, L., Conway, A., Lockhart, D.J., Davis, R.W., Brewer, B.J., and Fangman, W.L. (2001). Replication dynamics of the yeast genome. Science 294, 115-121.

Rathmell, W.K., and Chu, G. (1994). Involvement of the Ku autoantigen in the cellular response to DNA double-strand breaks. Proceedings of the National Academy of Sciences of the United States of America 91, 7623-7627.

Ray, A., and Runge, K.W. (1999a). Varying the number of telomere-bound proteins does not alter telomere length in tel1Delta cells. Proceedings of the National Academy of Sciences of the United States of America 96, 15044-15049.

Ray, A., and Runge, K.W. (1999b). The yeast telomere length counting machinery is sensitive to sequences at the telomere-nontelomere junction. Molecular and cellular biology 19, 31-45.

Ribeyre, C., and Shore, D. (2012). Anticheckpoint pathways at telomeres in yeast. Nat Struct Mol Biol 19, 307-U364.

Richard, P., and Manley, J.L. (2009). Transcription termination by nuclear RNA polymerases. Genes Dev 23, 1247-1269.

Roy, R., Meier, B., McAinsh, A.D., Feldmann, H.M., and Jackson, S.P. (2004). Separation-of-function mutants of yeast Ku80 reveal a Yku80p-Sir4p interaction involved in telomeric silencing. The Journal of biological chemistry $279,86-94$.

Sabourin, M., Tuzon, C.T., and Zakian, V.A. (2007). Telomerase and Tel1p preferentially associate with short telomeres in S. cerevisiae. Mol Cell 27, 550-561.

Sandhu, R., Sanford, S., Basu, S., Park, M., Pandya, U.M., Li, B., and Chakrabarti, K. (2013). A trans-spliced telomerase RNA dictates telomere synthesis in Trypanosoma brucei. Cell research 23, 537-551.

Schneider, C., Kudla, G., Wlotzka, W., Tuck, A., and Tollervey, D. (2012). Transcriptome-wide analysis of exosome targets. Mol Cell 48, 422-433.

Schneider, C., Leung, E., Brown, J., and Tollervey, D. (2009). The N-terminal PIN domain of the exosome subunit Rrp44 harbors endonuclease activity and tethers Rrp44 to the yeast core exosome. Nucleic Acids Res 37, 1127-1140.

Segref, A., Sharma, K., Doye, V., Hellwig, A., Huber, J., Luhrmann, R., and Hurt, E. (1997). Mex67p, a novel factor for nuclear mRNA export, binds to both poly $(A)(+)$ RNA and nuclear pores. Embo J 16 3256-3271. 
Seipelt, R.L., Zheng, B., Asuru, A., and Rymond, B.C. (1999). U1 snRNA is cleaved by RNase III and processed through an Sm site-dependent pathway. Nucleic acids research 27, 587-595.

Senger, B., Simos, G., Bischoff, F.R., Podtelejnikov, A., Mann, M., and Hurt, E. (1998). Mtr10p functions as a nuclear import receptor for the mRNA-binding protein Npl3p. The EMBO journal 17, 2196-2207.

Seto, A.G., Livengood, A.J., Tzfati, Y., Blackburn, E.H., and Cech, T.R. (2002). A bulged stem tethers Est1p to telomerase RNA in budding yeast. Genes Dev 16, 2800-2812.

Seto, A.G., Zaug, A.J., Sobel, S.G., Wolin, S.L., and Cech, T.R. (1999). Saccharomyces cerevisiae telomerase is an Sm small nuclear ribonucleoprotein particle. Nature 401, 177-180.

Shampay, J., Szostak, J.W., and Blackburn, E.H. (1984). DNA sequences of telomeres maintained in yeast. Nature 310, 154-157.

Shima, H., Suzuki, M., and Shinohara, M. (2005). Isolation and characterization of novel xrs2 mutations in Saccharomyces cerevisiae. Genetics 170, 71-85.

Sikorski, R.S., and Hieter, P. (1989). A System of Shuttle Vectors and Yeast Host Strains Designed for Efficient Manipulation of DNA in Saccharomyces-Cerevisiae. Genetics 122, 19-27.

Singer, M.S., and Gottschling, D.E. (1994). TLC1: template RNA component of Saccharomyces cerevisiae telomerase. Science 266, 404-409.

Skruzny, M., Schneider, C., Racz, A., Weng, J., Tollervey, D., and Hurt, E. (2009). An endoribonuclease functionally linked to perinuclear mRNP quality control associates with the nuclear pore complexes. Plos Biol 7, e8.

Slomovic, S., and Schuster, G. (2011). Exonucleases and endonucleases involved in polyadenylation-assisted RNA decay. Wiley interdisciplinary reviews. RNA 2, 106-123.

Snay-Hodge, C.A., Colot, H.V., Goldstein, A.L., and Cole, C.N. (1998). Dbp5p/Rat8p is a yeast nuclear pore-associated DEAD-box protein essential for RNA export. The EMBO journal 17, 2663-2676.

Stade, K., Ford, C.S., Guthrie, C., and Weis, K. (1997). Exportin 1 (Crm1p) is an essential nuclear export factor. Cell 90, 1041-1050.

Stage-Zimmermann, T., Schmidt, U., and Silver, P.A. (2000). Factors affecting nuclear export of the 60S ribosomal subunit in vivo. Molecular biology of the cell 11, 3777-3789.

Stansfield, I., Kushnirov, V.V., Jones, K.M., and Tuite, M.F. (1997). A conditional-lethal translation termination defect in a sup45 mutant of the yeast Saccharomyces cerevisiae. European journal of biochemistry / FEBS 245, 557-563.

Steinmetz, E.J., Conrad, N.K., Brow, D.A., and Corden, J.L. (2001). RNA-binding protein Nrd1 directs poly(A)-independent 3'-end formation of RNA polymerase II transcripts. Nature 413, 327-331.

Stellwagen, A.E., Haimberger, Z.W., Veatch, J.R., and Gottschling, D.E. (2003). Ku interacts with telomerase RNA to promote telomere addition at native and broken chromosome ends. Genes Dev 17, 2384-2395.

Suyama, M., Doerks, T., Braun, I.C., Sattler, M., Izaurralde, E., and Bork, P. (2000). Prediction of structural domains of TAP reveals details of its interaction with p15 and nucleoporins. Embo Rep 1, 53-58.

Synowsky, S.A., van Wijk, M., Raijmakers, R., and Heck, A.J.R. (2009). Comparative Multiplexed Mass Spectrometric Analyses of Endogenously Expressed Yeast Nuclear and Cytoplasmic Exosomes. J Mol Biol 385, 1300-1313.

Szostak, J.W., and Blackburn, E.H. (1982). Cloning yeast telomeres on linear plasmid vectors. Cell 29, 245-255. 
Taccioli, G.E., Gottlieb, T.M., Blunt, T., Priestley, A., Demengeot, J., Mizuta, R., Lehmann, A.R., Alt, F.W., Jackson, S.P., and Jeggo, P.A. (1994). Ku80: product of the XRCC5 gene and its role in DNA repair and $V(D) J$ recombination. Science 265, 1442-1445.

Taggart, A.K., Teng, S.C., and Zakian, V.A. (2002). Est1p as a cell cycle-regulated activator of telomere-bound telomerase. Science 297, 1023-1026.

Talley, J.M., DeZwaan, D.C., Maness, L.D., Freeman, B.C., and Friedman, K.L. (2011). Stimulation of yeast telomerase activity by the ever shorter telomere 3 (Est3) subunit is dependent on direct interaction with the catalytic protein Est2. The Journal of biological chemistry 286, 26431-26439.

Taura, T., Krebber, H., and Silver, P.A. (1998). A member of the Ran-binding protein family, Yrb2p, is involved in nuclear protein export. Proceedings of the National Academy of Sciences of the United States of America 95, 7427-7432.

Tieg, B., and Krebber, H. (2013). Dbp5 - from nuclear export to translation. Biochimica et biophysica acta $1829,791-798$.

Torchet, C., Bousquet-Antonelli, C., Milligan, L., Thompson, E., Kufel, J., and Tollervey, D. (2002). Processing of 3 '-extended read-through transcripts by the exosome can generate functional mRNAs. Mol Cell 9, 1285-1296.

Tran, P.T., Erdeniz, N., Dudley, S., and Liskay, R.M. (2002). Characterization of nuclease-dependent functions of Exo1p in Saccharomyces cerevisiae. DNA repair 1, 895-912.

Tsukamoto, Y., Taggart, A.K., and Zakian, V.A. (2001). The role of the Mre11-Rad50-Xrs2 complex in telomerase- mediated lengthening of Saccharomyces cerevisiae telomeres. Current biology : CB 11, 1328-1335.

Tudek, A., Porrua, O., Kabzinski, T., Lidschreiber, M., Kubicek, K., Fortova, A., Lacroute, F., Vanacova, S., Cramer, P., Stefl, R., et al. (2014). Molecular basis for coordinating transcription termination with noncoding RNA degradation. Mol Cell 55, 467-481.

Tuzon, C.T., Wu, Y., Chan, A., and Zakian, V.A. (2011). The Saccharomyces cerevisiae telomerase subunit Est3 binds telomeres in a cell cycle- and Est1-dependent manner and interacts directly with Est1 in vitro. Plos Genet 7, e1002060.

Van Hoof, A., Lennertz, P., and Parker, R. (2000a). Yeast exosome mutants accumulate 3 '-extended polyadenylated forms of U4 small nuclear RNA and small nucleolar RNAs. Mol Cell Biol 20, 441-452.

van Hoof, A., Staples, R.R., Baker, R.E., and Parker, R. (2000b). Function of the Ski4p (Csl4p) and Ski7p proteins in 3 '-to-5 ' degradation of mRNA. Mol Cell Biol 20, 8230-8243.

Vanacova, S., Wolf, J., Martin, G., Blank, D., Dettwiler, S., Friedlein, A., Langen, H., Keith, G., and Keller, W. (2005). A new yeast poly(A) polymerase complex involved in RNA quality control. Plos Biol 3, 986-997.

Vasiljeva, L., Kim, M., Mutschler, H., Buratowski, S., and Meinhart, A. (2008). The Nrd1-Nab3-Sen1 termination complex interacts with the Ser5-phosphorylated RNA polymerase II C-terminal domain. Nat Struct Mol Biol 15, 795-804.

Vega, L.R., Phillips, J.A., Thornton, B.R., Benanti, J.A., Onigbanjo, M.T., Toczyski, D.P., and Zakian, V.A. (2007). Sensitivity of yeast strains with long G-tails to levels of telomere-bound telomerase. Plos Genet 3, e105.

Virta-Pearlman, V., Morris, D.K., and Lundblad, V. (1996). Est1 has the properties of a single-stranded telomere end-binding protein. Genes Dev 10, 3094-3104.

Vodenicharov, M.D., and Wellinger, R.J. (2007). The cell division cycle puts up with unprotected 
telomeres: cell cycle regulated telomere uncapping as a means to achieve telomere homeostasis. Cell Cycle 6, 1161-1167.

Wang, S.S., and Zakian, V.A. (1990). Sequencing of Saccharomyces telomeres cloned using T4 DNA polymerase reveals two domains. Molecular and cellular biology 10, 4415-4419.

Watson, J.D. (1972). Origin of Concatemeric T7 DNA. Nature-New Biol 239, 197-\&.

Weir, J.R., Bonneau, F., Hentschel, J., and Conti, E. (2010). Structural analysis reveals the characteristic features of Mtr4, a DEXH helicase involved in nuclear RNA processing and surveillance. Proceedings of the National Academy of Sciences of the United States of America 107, 12139-12144.

Weirich, C.S., Erzberger, J.P., Berger, J.M., and Weis, K. (2004). The N-terminal domain of Nup159 forms a beta-propeller that functions in mRNA export by tethering the helicase Dbp5 to the nuclear pore. Mol Cell 16, 749-760.

Wellinger, R.J., Wolf, A.J., and Zakian, V.A. (1993a). Origin activation and formation of single-strand TG1-3 tails occur sequentially in late $S$ phase on a yeast linear plasmid. Molecular and cellular biology 13, 4057-4065.

Wellinger, R.J., Wolf, A.J., and Zakian, V.A. (1993b). Saccharomyces telomeres acquire single-strand TG1-3 tails late in S phase. Cell 72, 51-60.

Wellinger, R.J., and Zakian, V.A. (2012). Everything you ever wanted to know about Saccharomyces cerevisiae telomeres: beginning to end. Genetics 191, 1073-1105.

Will, C.L., and Luhrmann, R. (2001). Spliceosomal UsnRNP biogenesis, structure and function. Current opinion in cell biology 13, 290-301.

Windgassen, M., and Krebber, H. (2003). Identification of Gbp2 as a novel poly(A)+ RNA-binding protein involved in the cytoplasmic delivery of messenger RNAs in yeast. Embo Rep 4, 278-283.

Winston, F., Dollard, C., and Ricuperohovasse, S.L. (1995). Construction of a Set of Convenient Saccharomyces-Cerevisiae Strains That Are Isogenic to S288c. Yeast 11, 53-55.

Wotton, D., and Shore, D. (1997). A novel Rap1p-interacting factor, Rif2p, cooperates with Rif1p to regulate telomere length in Saccharomyces cerevisiae. Genes Dev 11, 748-760.

Wright, J.H., and Zakian, V.A. (1995). Protein-DNA interactions in soluble telosomes from Saccharomyces cerevisiae. Nucleic acids research 23, 1454-1460.

Wu, Y., and Zakian, V.A. (2011). The telomeric Cdc13 protein interacts directly with the telomerase subunit Est1 to bring it to telomeric DNA ends in vitro. Proceedings of the National Academy of Sciences of the United States of America 108, 20362-20369.

Wyers, F., Rougemaille, M., Badis, G., Rousselle, J.C., Dufour, M.E., Boulay, J., Regnault, B., Devaux, F., Namane, A., Seraphin, B., et al. (2005). Cryptic Pol II transcripts are degraded by a nuclear quality control pathway involving a new poly(A) polymerase. Cell 121, 725-737.

Yao, W., Roser, D., Kohler, A., Bradatsch, B., Bassler, J., and Hurt, E. (2007). Nuclear export of ribosomal 60S subunits by the general mRNA export receptor Mex67-Mtr2. Mol Cell 26, 51-62.

Yi, X., Tesmer, V.M., Savre-Train, I., Shay, J.W., and Wright, W.E. (1999). Both transcriptional and posttranscriptional mechanisms regulate human telomerase template RNA levels. Molecular and cellular biology 19, 3989-3997.

Zappulla, D.C., and Cech, T.R. (2004). Yeast telomerase RNA: a flexible scaffold for protein subunits. Proceedings of the National Academy of Sciences of the United States of America 101, 10024-10029.

Zappulla, D.C., Goodrich, K., and Cech, T.R. (2005). A miniature yeast telomerase RNA functions in vivo 
and reconstitutes activity in vitro. Nat Struct Mol Biol 12, 1072-1077.

Zenklusen, D., Vinciguerra, P., Strahm, Y., and Stutz, F. (2001). The yeast hnRNP-Like proteins Yra1p and Yra2 $p$ participate in mRNA export through interaction with Mex67p. Molecular and cellular biology 21, 4219-4232.

Zenklusen, D., Vinciguerra, P., Wyss, J.C., and Stutz, F. (2002). Stable mRNP formation and export require cotranscriptional recruitment of the mRNA export factors Yra1p and Sub2p by Hpr1p. Molecular and cellular biology 22, 8241-8253. 


\section{Acknowledgments}

Filling in the last word in this thesis is ending of a stage of my life. Experiences during these years will be a precious gift which will lead me and accompany with me. Countless thanks to those people, who instruct me, encourage me and move me.

I would like to send my greatest gratefulness to Prof. Dr. Heike Krebber, for guiding me into a new world and for always inspiring me on the way of this beautiful exploration.

I would also like to express my sincere gratitude to PD Dr. Wilfried Kramer, for sharing his excellent and exhaustive minds on the aspects of both science and life.

I would like to thank Prof. Dr. Holger Bastians for his heartfelt support on my future investigation. I would like to thank Prof. Dr. Gerhard Braus, Prof. Dr. Ralf Ficner, Prof. Dr. Stefanie Pöggeler, Prof. Dr. Kai Heimel for spending time on evaluating my work.

I would like to thank Dr. Alexandra Hackmann, Daniel Becker and Ulla-Maria Schneider for their helpful collaborations and selfless contributions to my work.

I would like to thank all of my dear lab colleagues, Dr. Claudia Baierlein, Lysann Henker, Bettina Tieg, Gesa Zander, Lena Oldehaver, Angelika Löffers and Angela Bindseil for sharing our emotions, for treating me like a real family member and for bringing me endless happy memories.

I am also grateful to those, who have ever helped me and encouraged me: members of group of Prof. Dr. Holger Bastians, Dr. Norman Ertych, Dr. Aline Stolz, Dr. Phillip Kaestner, Dr. Makus Becker, Sina Lüddecke and Katharina Berger.

I would also like to express lots of gratitude but also apologies to my parents and my in-laws, Fengming Ma, Guoping $\mathrm{Wu}$, Tang $\mathrm{Li}$ and $\mathrm{Li} \mathrm{Li}$, for their unconditional love and support even by disappointment only, for being not able to fulfill my duty as a son for too long time and for never doing enough to repay them.

The last, but not the least part is dedicated to my wife, Sangmeng Li. There are many thanks beyond words to her for only encouraging me but never pressing me. It is the luckiest thing to meet her and know her in my life and it is the happiest thing to share the rest of my life with her. 


\section{Curriculum Vitae}

Year2011-2015 Georg-August-University Göttingen, Germany

Dept. Molecular Genetics, Institute of Microbiology and Genetics

Ph.D. Candidate in group of Prof. Dr. Heike Krebber

Year2009-2011

Philipps-University Marburg, Germany

IMT (Institute of Molecular Biology and Tumor Research)

Ph.D. Candidate in group of Prof. Dr. Heike Krebber

Year2003-2009

Eberhard-Karls University Tübingen, Germany

Diploma Biologie (Dipl.-Biol.)

Year2008-2009 The Max Planck Institute for Developmental Biology, Tübingen Germany Diploma thesis in group of Prof. Dr. Ingrid Lohmann

Year1996-2000 Huazhong Agriculture University in Wuhan, Hubei province, China Bachelor of Science (B.Sc) in microbiology

Year1984-1996 Basic Education in Shuiguohu School in Wuhan, Hubei province, China 